\title{
Synthesis, Electrochemical Characterization, and Linear Free Energy Relationship of 1,3-
}

\section{Diphenyl-6-alkyl/arylfulvenes}

Nicholas P. Godman, ${ }^{\dagger}$ Sonya K. Adas, ${ }^{\dagger}$ Karl M. Hellwig, ${ }^{\dagger}$ David W. Ball, ${ }^{\S}$ Gary J. Balaich, ${ }^{* \dagger}$ Scott T. Iacono* ${ }^{\dagger}$

$\uparrow$ Department of Chemistry and Chemistry Research Center, United States Air Force Academy, Colorado Springs, Colorado 80840,

United States

$\S$ Department of Chemistry, Cleveland State University, Cleveland, Ohio 44415, United States

*gary.balaich@usafa.edu, scott.iacono@usafa.edu 


\section{Table of Contents}

\section{Contents}

\section{Computational Data}

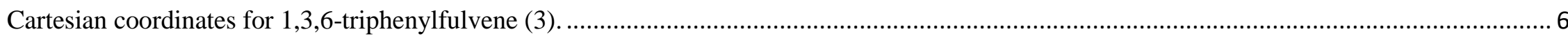

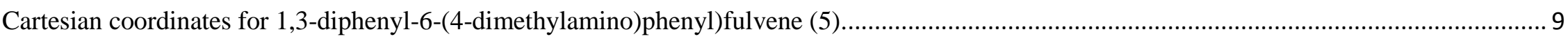

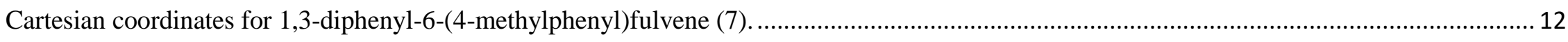

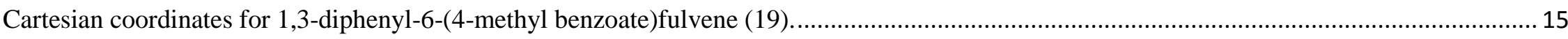

Cartesian coordinates for 1,3-diphenyl-6-4-(trifluoromethyl)phenyl]fulvene (20). .............................................................................. 18

Cartesian coordinates for 1,3-diphenyl-6-(4-trimethylammoniu)phenyl)fulvene (21) ………................................................................... 21

\section{Cyclic Voltammograms}

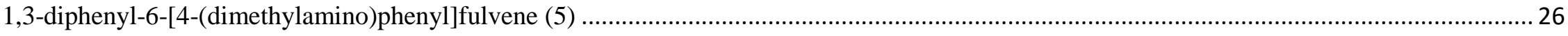

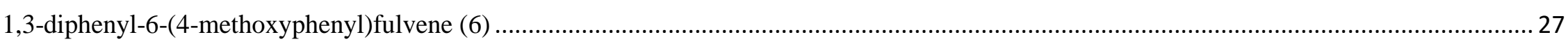

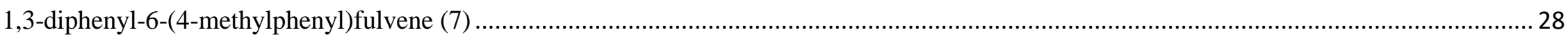

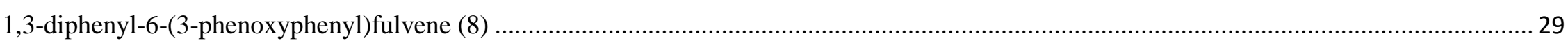

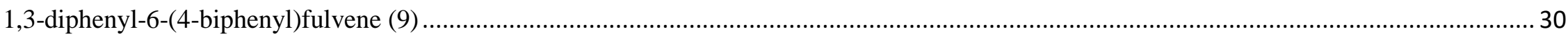

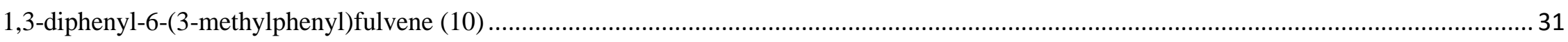

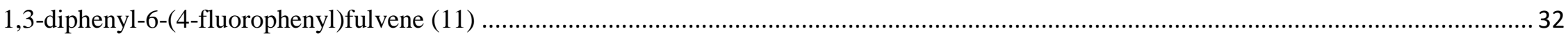


1,3-diphenyl-6-(3-methoxyphenyl)fulvene (12)

1,3-diphenyl-6-(3-vinylphenyl)fulvene (13)........

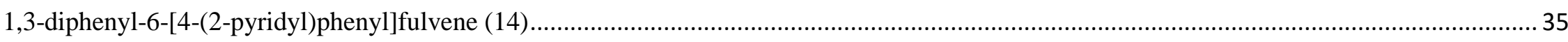

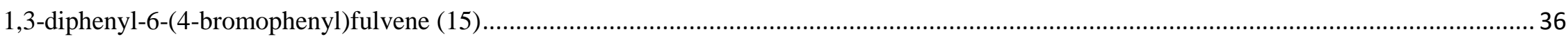

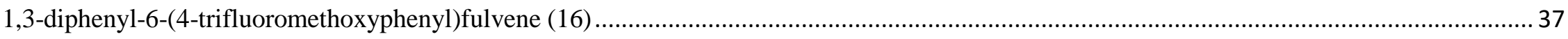

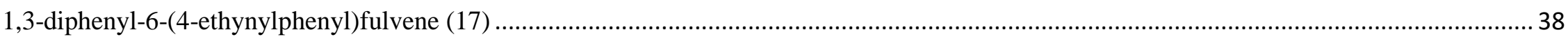

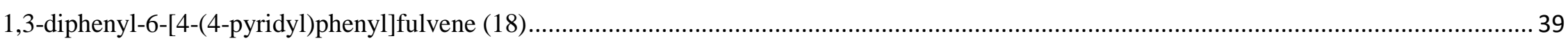

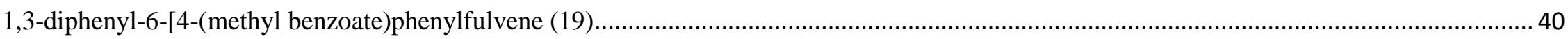

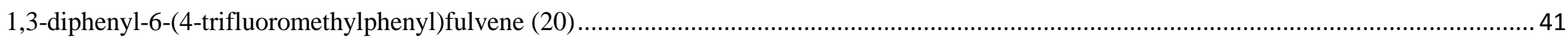

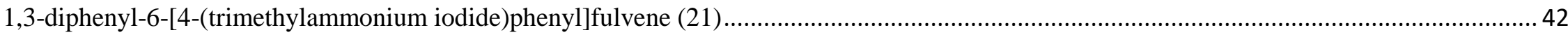

\section{NMR Spectra}

1,3-diphenyl-5-benzylcyclopentadiene (4) ${ }^{1} \mathrm{H}-\mathrm{NMR}\left(400 \mathrm{MHz}, \mathrm{CDCl}_{3}\right)$

1,3-diphenyl-5-benzylcyclopentadiene (4) ${ }^{13} \mathrm{C}-\mathrm{NMR}\left(100 \mathrm{MHz}, \mathrm{CDCl}_{3}\right)$

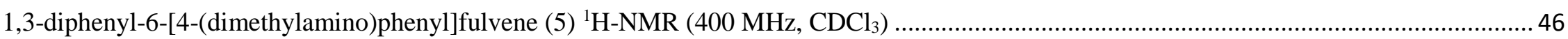

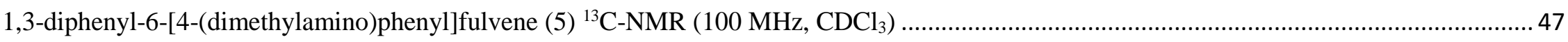

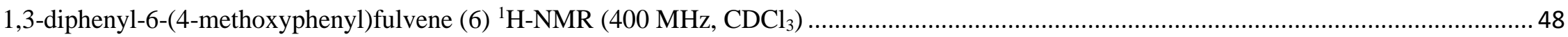

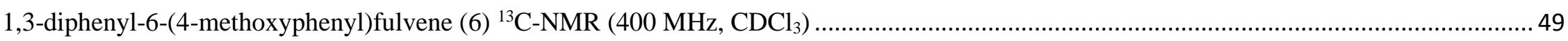

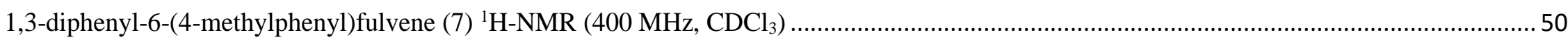

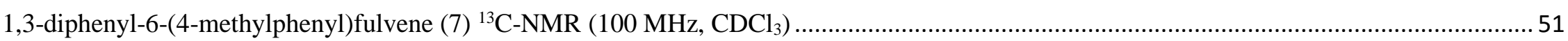

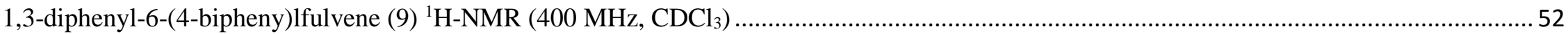

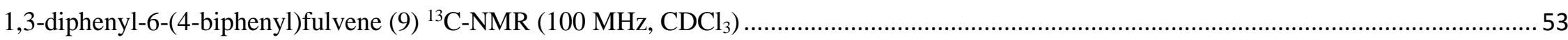


1,3-diphenyl-6-(3-methylphenyl)lfulvene (10) ${ }^{13} \mathrm{C}-\mathrm{NMR}\left(100 \mathrm{MHz}, \mathrm{CDCl}_{3}\right)$.....

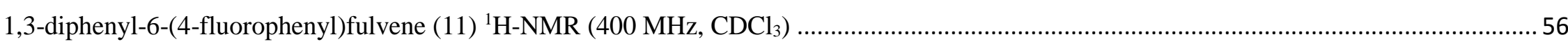

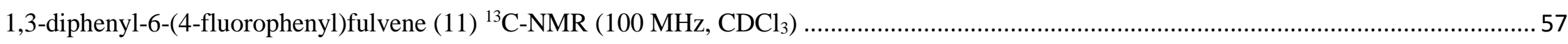

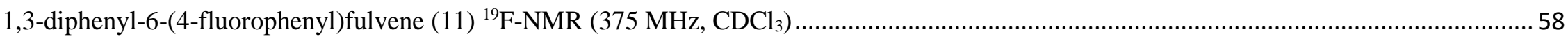

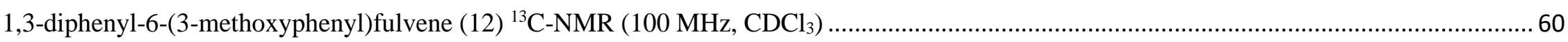

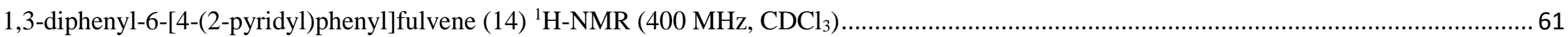

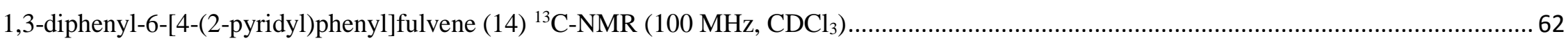

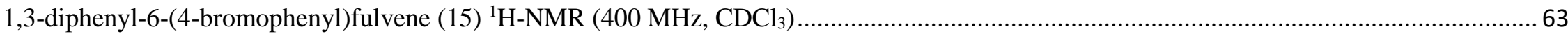

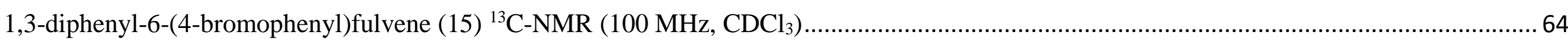

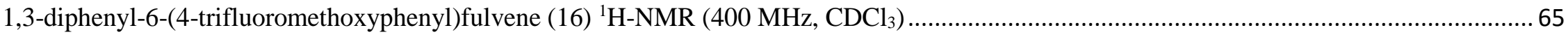

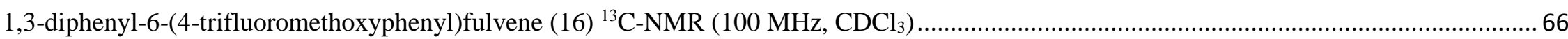

1,3-diphenyl-6-(4-trifluoromethoxyphenyl)fulvene (16) ${ }^{19} \mathrm{~F}-\mathrm{NMR}\left(376 \mathrm{MHz}, \mathrm{CDCl}_{3}\right)$.......................................................................67

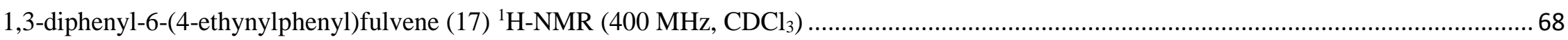

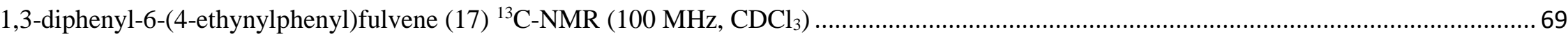

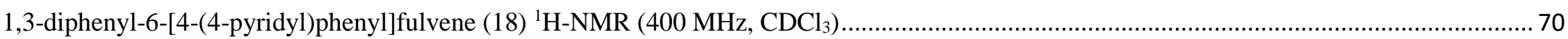

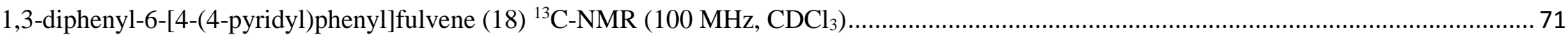

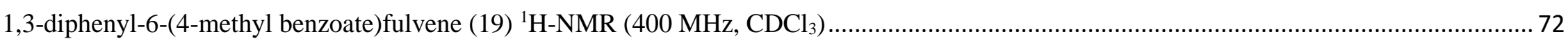

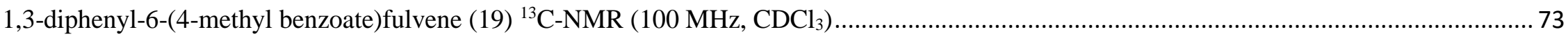

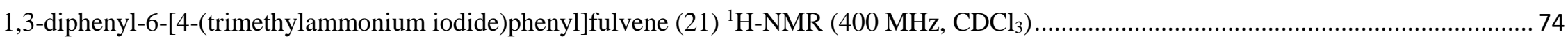

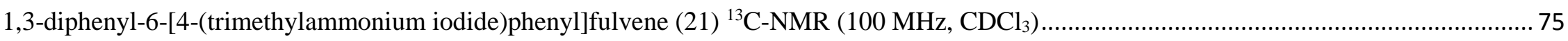

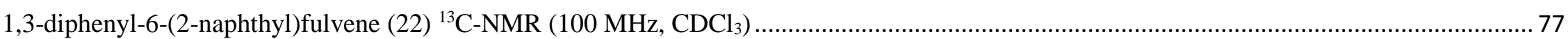

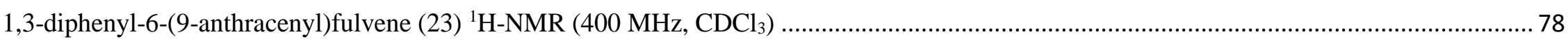

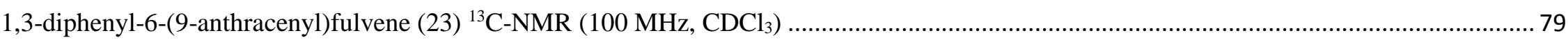


Computational Data 
Cartesian coordinates for 1,3,6-triphenylfulvene (3).

\begin{tabular}{|c|c|c|c|c|c|}
\hline \multirow{2}{*}{$\begin{array}{l}\text { Center } \\
\text { Number }\end{array}$} & \multirow{2}{*}{$\begin{array}{l}\text { Atomic } \\
\text { Number }\end{array}$} & \multirow{2}{*}{$\begin{array}{l}\text { Atomic } \\
\text { Type }\end{array}$} & \multicolumn{3}{|c|}{ Coordinates (Angstroms) } \\
\hline & & & $\mathrm{X}$ & Y & Z \\
\hline 1 & 6 & 0 & -0.880228 & 0.703040 & 0.087956 \\
\hline 2 & 6 & 0 & 0.487416 & 0.198556 & 0.061951 \\
\hline 3 & 6 & 0 & 0.379442 & -1.285979 & 0.026484 \\
\hline 4 & 6 & 0 & -0.943466 & -1.588916 & -0.030329 \\
\hline 5 & 6 & 0 & -1.743252 & -0.354942 & 0.029201 \\
\hline 6 & 1 & 0 & -1.154324 & 1.743696 & 0.147964 \\
\hline 7 & 6 & 0 & 1.663919 & 0.875443 & 0.007915 \\
\hline 8 & 1 & 0 & 2.567634 & 0.272944 & 0.040397 \\
\hline 9 & 6 & 0 & 1.909054 & 2.315312 & -0.069419 \\
\hline 10 & 6 & 0 & 3.115860 & 2.813478 & 0.457310 \\
\hline 11 & 6 & 0 & 1.028937 & 3.228479 & -0.678930 \\
\hline 12 & 6 & 0 & 3.409276 & 4.171804 & 0.425171 \\
\hline 13 & 1 & 0 & 3.820211 & 2.121722 & 0.907354 \\
\hline 14 & 6 & 0 & 1.329575 & 4.585623 & -0.721277 \\
\hline
\end{tabular}




\begin{tabular}{|c|c|c|c|c|c|}
\hline 15 & 1 & 0 & 0.128541 & 2.866311 & -1.156716 \\
\hline 16 & 6 & 0 & 2.513729 & 5.065565 & -0.161309 \\
\hline 17 & 1 & 0 & 4.339402 & 4.532822 & 0.849379 \\
\hline 18 & 1 & 0 & 0.643008 & 5.271008 & -1.205656 \\
\hline 19 & 1 & 0 & 2.743786 & 6.124333 & -0.196739 \\
\hline 20 & 6 & 0 & -3.210222 & -0.310227 & 0.041757 \\
\hline 21 & 6 & 0 & -3.969715 & -1.379001 & -0.463240 \\
\hline 22 & 6 & 0 & -3.901316 & 0.803210 & 0.552676 \\
\hline 23 & 6 & 0 & -5.361332 & -1.332386 & -0.469019 \\
\hline 24 & 1 & 0 & -3.466916 & -2.247392 & -0.872148 \\
\hline 25 & 6 & 0 & -5.290061 & 0.849950 & 0.546344 \\
\hline 26 & 1 & 0 & -3.341325 & 1.629339 & 0.975289 \\
\hline 27 & 6 & 0 & -6.029036 & -0.218053 & 0.034288 \\
\hline 28 & 1 & 0 & -5.924488 & -2.168024 & -0.869736 \\
\hline 29 & 1 & 0 & -5.800014 & 1.717303 & 0.951043 \\
\hline 30 & 1 & 0 & -7.112527 & -0.182397 & 0.033193 \\
\hline 31 & 1 & 0 & -1.352725 & -2.589674 & -0.040735 \\
\hline 32 & 6 & 0 & 1.493853 & -2.249966 & 0.038207 \\
\hline 33 & 6 & 0 & 2.506751 & -2.191446 & 1.010258 \\
\hline
\end{tabular}




$\begin{array}{llllll}34 & 6 & 0 & 1.549107 & -3.282608 & -0.911854 \\ 35 & 6 & 0 & 3.536447 & -3.129093 & 1.027867 \\ 36 & 1 & 0 & 2.465591 & -1.424837 & 1.775547 \\ 37 & 6 & 0 & 2.576506 & -4.221906 & -0.891271 \\ 38 & 1 & 0 & 0.785524 & -3.332017 & -1.679886 \\ 39 & 6 & 0 & 3.577064 & -4.147633 & 0.076848 \\ 40 & 1 & 0 & 4.302376 & -3.070584 & 1.793545 \\ 41 & 1 & 0 & 2.600565 & -5.008412 & -1.637646 \\ 42 & 1 & 0 & 4.378966 & -4.877085 & 0.091500\end{array}$

Total electronic energy (B3LYP/6-311+G(d,p)) = -925.59754 hartrees 
Cartesian coordinates for 1,3-diphenyl-6-(4-dimethylamino)phenyl)fulvene (5).

\begin{tabular}{|c|c|c|c|c|c|}
\hline \multirow{2}{*}{$\begin{array}{l}\text { Center } \\
\text { Number }\end{array}$} & \multirow{2}{*}{$\begin{array}{l}\text { Atomic } \\
\text { Number }\end{array}$} & \multirow{2}{*}{$\begin{array}{l}\text { Atomic } \\
\text { Type }\end{array}$} & \multicolumn{3}{|c|}{ Coordinates (Angstroms) } \\
\hline & & & $\mathrm{X}$ & Y & Z \\
\hline 1 & 6 & 0 & 0.629487 & -0.918250 & 0.093803 \\
\hline 2 & 6 & 0 & -0.193455 & 0.279976 & 0.057669 \\
\hline 3 & 6 & 0 & 0.751967 & 1.425057 & 0.031132 \\
\hline 4 & 6 & 0 & 2.011433 & 0.908641 & -0.010910 \\
\hline 5 & 6 & 0 & 1.949701 & -0.554498 & 0.049548 \\
\hline 6 & 1 & 0 & 0.258964 & -1.928595 & 0.150105 \\
\hline 7 & 6 & 0 & -1.548895 & 0.420848 & -0.024942 \\
\hline 8 & 1 & 0 & -1.911976 & 1.445169 & -0.003947 \\
\hline 9 & 6 & 0 & -2.609081 & -0.562453 & -0.130893 \\
\hline 10 & 6 & 0 & -3.927217 & -0.161559 & 0.167591 \\
\hline 11 & 6 & 0 & -2.445078 & -1.896323 & -0.555565 \\
\hline 12 & 6 & 0 & -5.001492 & -1.030846 & 0.108130 \\
\hline 13 & 1 & 0 & -4.106661 & 0.866693 & 0.465222 \\
\hline
\end{tabular}




\begin{tabular}{|c|c|c|c|c|c|}
\hline 14 & 6 & 0 & -3.508833 & -2.776588 & -0.632477 \\
\hline 15 & 1 & 0 & -1.472193 & -2.241188 & -0.877157 \\
\hline 16 & 6 & 0 & -4.821650 & -2.380081 & -0.277599 \\
\hline 17 & 1 & 0 & -5.985434 & -0.657969 & 0.355540 \\
\hline 18 & 1 & 0 & -3.321168 & -3.779517 & -0.989717 \\
\hline 19 & 6 & 0 & 3.117788 & -1.442821 & 0.074780 \\
\hline 20 & 6 & 0 & 4.359452 & -1.018692 & -0.428638 \\
\hline 21 & 6 & 0 & 3.034139 & -2.746694 & 0.596184 \\
\hline 22 & 6 & 0 & 5.466106 & -1.863662 & -0.422127 \\
\hline 23 & 1 & 0 & 4.454797 & -0.023258 & -0.846047 \\
\hline 24 & 6 & 0 & 4.138157 & -3.591051 & 0.600910 \\
\hline 25 & 1 & 0 & 2.097337 & -3.090756 & 1.019062 \\
\hline 26 & 6 & 0 & 5.362207 & -3.155052 & 0.090773 \\
\hline 27 & 1 & 0 & 6.411260 & -1.512549 & -0.821755 \\
\hline 28 & 1 & 0 & 4.047984 & -4.590091 & 1.013575 \\
\hline 29 & 1 & 0 & 6.224053 & -3.812704 & 0.098650 \\
\hline 30 & 1 & 0 & 2.923093 & 1.490170 & -0.007409 \\
\hline 31 & 6 & 0 & 0.407552 & 2.857718 & 0.045975 \\
\hline 32 & 6 & 0 & -0.466257 & 3.395594 & 1.006240 \\
\hline
\end{tabular}




$\begin{array}{llllll}33 & 6 & 0 & 0.983523 & 3.737293 & -0.885556 \\ 34 & 6 & 0 & -0.754060 & 4.758174 & 1.030368 \\ 35 & 1 & 0 & -0.894564 & 2.743028 & 1.758370 \\ 36 & 6 & 0 & 0.699712 & 5.100069 & -0.857987 \\ 37 & 1 & 0 & 1.646385 & 3.337378 & -1.644520 \\ 38 & 6 & 0 & -0.173503 & 5.617112 & 0.098163 \\ 39 & 1 & 0 & -1.423714 & 5.151821 & 1.787489 \\ 40 & 1 & 0 & 1.154877 & 5.758429 & -1.590039 \\ 41 & 1 & 0 & -0.397185 & 6.677819 & 0.118217 \\ 42 & 7 & 0 & -5.877187 & -3.266401 & -0.317311 \\ 43 & 6 & 0 & -7.236512 & -2.785873 & -0.121227 \\ 44 & 1 & 0 & -7.918846 & -3.634295 & -0.136651 \\ 45 & 1 & 0 & -7.343565 & -2.292452 & 0.849178 \\ 46 & 1 & 0 & -7.548545 & -2.079128 & -0.902533 \\ 47 & 1 & 0 & -5.687815 & -4.600154 & -0.867631 \\ 48 & 1 & 0 & -5.427112 & -4.581690 & -1.934874 \\ 49 & 1 & 0 & -5.138299 & -0.331871 \\ 50 & 1 & 0.165862 & -0.752090\end{array}$

Total electronic energy (B $3 \mathrm{LYP} / 6-311+\mathrm{G}(\mathrm{d}, \mathrm{p}))=-1059.60401$ hartrees 
Cartesian coordinates for 1,3-diphenyl-6-(4-methylphenyl)fulvene (7).

\begin{tabular}{|c|c|c|c|c|c|}
\hline \multirow{2}{*}{$\begin{array}{l}\text { Center } \\
\text { Number }\end{array}$} & \multirow{2}{*}{$\begin{array}{l}\text { Atomic } \\
\text { Number }\end{array}$} & \multirow{2}{*}{$\begin{array}{l}\text { Atomic } \\
\text { Type }\end{array}$} & \multicolumn{3}{|c|}{ Coordinates (Angstroms) } \\
\hline & & & $\mathrm{x}$ & Y & Z \\
\hline 1 & 6 & 0 & 0.773377 & 0.813019 & -0.092315 \\
\hline 2 & 6 & 0 & -0.509115 & 0.122352 & -0.044902 \\
\hline 3 & 6 & 0 & -0.193631 & -1.331926 & -0.020353 \\
\hline 4 & 6 & 0 & 1.160036 & -1.447386 & 0.011221 \\
\hline 5 & 6 & 0 & 1.777274 & -0.114197 & -0.055438 \\
\hline 6 & 1 & 0 & 0.899868 & 1.881847 & -0.149555 \\
\hline 7 & 6 & 0 & -1.769235 & 0.626017 & 0.035600 \\
\hline 8 & 1 & 0 & -2.576624 & -0.101106 & 0.016789 \\
\hline 9 & 6 & 0 & -2.220229 & 2.012043 & 0.125758 \\
\hline 10 & 6 & 0 & -3.510053 & 2.326546 & -0.340371 \\
\hline 11 & 6 & 0 & -1.465146 & 3.055359 & 0.692205 \\
\hline 12 & 6 & 0 & -3.998646 & 3.625979 & -0.295269 \\
\hline 13 & 1 & 0 & -4.129280 & 1.536942 & -0.753358 \\
\hline
\end{tabular}




\begin{tabular}{|c|c|c|c|c|c|}
\hline 14 & 6 & 0 & -1.964955 & 4.350092 & 0.746314 \\
\hline 15 & 1 & 0 & -0.501014 & 2.840990 & 1.133266 \\
\hline 16 & 6 & 0 & -3.232969 & 4.666536 & 0.242290 \\
\hline 17 & 1 & 0 & -4.994144 & 3.834702 & -0.673750 \\
\hline 18 & 1 & 0 & -1.364467 & 5.130218 & 1.203637 \\
\hline 19 & 6 & 0 & 3.223029 & 0.136059 & -0.094227 \\
\hline 20 & 6 & 0 & 4.133077 & -0.810217 & 0.406290 \\
\hline 21 & 6 & 0 & 3.742822 & 1.329407 & -0.627060 \\
\hline 22 & 6 & 0 & 5.504274 & -0.568947 & 0.387412 \\
\hline 23 & 1 & 0 & 3.763305 & -1.735916 & 0.831145 \\
\hline 24 & 6 & 0 & 5.111341 & 1.570501 & -0.645292 \\
\hline 25 & 1 & 0 & 3.065467 & 2.063561 & -1.047643 \\
\hline 26 & 6 & 0 & 6.001031 & 0.622284 & -0.137307 \\
\hline 27 & 1 & 0 & 6.185535 & -1.312882 & 0.785533 \\
\hline 28 & 1 & 0 & 5.487892 & 2.496423 & -1.066103 \\
\hline 29 & 1 & 0 & 7.068716 & 0.809285 & -0.155673 \\
\hline 30 & 1 & 0 & 1.705179 & -2.381220 & 0.006660 \\
\hline 31 & 6 & 0 & -1.161634 & -2.442944 & -0.023958 \\
\hline 32 & 6 & 0 & -2.189012 & -2.518133 & -0.979659 \\
\hline
\end{tabular}




$\begin{array}{llllll}33 & 6 & 0 & -1.055353 & -3.482694 & 0.913925 \\ 34 & 6 & 0 & -3.076910 & -3.591036 & -0.993364 \\ 35 & 1 & 0 & -2.268869 & -1.745795 & -1.736039 \\ 36 & 6 & 0 & -1.940849 & -4.557034 & 0.897263 \\ 37 & 1 & 0 & -0.278934 & -3.432329 & 1.668947 \\ 38 & 6 & 0 & -2.958085 & -4.614714 & -0.054526 \\ 39 & 1 & -3.856168 & -3.633202 & -1.746610 \\ 40 & 1 & 0 & -1.841379 & -5.346509 & 1.634225 \\ 41 & 1 & 0 & -3.649580 & -5.449637 & -0.065831 \\ 42 & 6 & 0 & -3.751138 & 6.082239 & 0.272634 \\ 43 & 1 & 0 & -3.416287 & 6.638744 & -0.610016 \\ 44 & 1 & 0 & -4.843054 & 6.107799 & 0.280550 \\ 45 & 1 & 0 & -3.390830 & 6.620888 & 1.152361\end{array}$

Total electronic energy (B3LYP/6-311+G $(\mathrm{d}, \mathrm{p}))=-964.92569$ hartrees 
Cartesian coordinates for 1,3-diphenyl-6-(4-methyl benzoate)fulvene (19).

\begin{tabular}{|c|c|c|c|c|c|}
\hline \multirow{2}{*}{$\begin{array}{l}\text { Center } \\
\text { Number }\end{array}$} & \multirow{2}{*}{$\begin{array}{l}\text { Atomic } \\
\text { Number }\end{array}$} & \multirow{2}{*}{$\begin{array}{l}\text { Atomic } \\
\text { Type }\end{array}$} & \multicolumn{3}{|c|}{ Coordinates (Angstroms) } \\
\hline & & & $\mathrm{x}$ & Y & Z \\
\hline 1 & 6 & 0 & -0.767050 & -0.936969 & -0.122369 \\
\hline 2 & 6 & 0 & -0.442781 & 0.483539 & -0.063412 \\
\hline 3 & 6 & 0 & -1.746326 & 1.204452 & -0.011319 \\
\hline 4 & 6 & 0 & -2.721461 & 0.260898 & 0.027528 \\
\hline 5 & 6 & 0 & -2.123935 & -1.082525 & -0.063397 \\
\hline 6 & 1 & 0 & -0.045934 & -1.734011 & -0.201362 \\
\hline 7 & 6 & 0 & 0.765914 & 1.099133 & 0.003497 \\
\hline 8 & 1 & 0 & 0.755760 & 2.185494 & -0.002859 \\
\hline 9 & 6 & 0 & 2.104294 & 0.513414 & 0.061818 \\
\hline 10 & 6 & 0 & 3.182024 & 1.259907 & -0.454009 \\
\hline 11 & 6 & 0 & 2.387150 & -0.736477 & 0.643671 \\
\hline 12 & 6 & 0 & 4.474745 & 0.760544 & -0.438613 \\
\hline 13 & 1 & 0 & 2.990543 & 2.237338 & -0.883387 \\
\hline
\end{tabular}




\begin{tabular}{|c|c|c|c|c|c|}
\hline 14 & 6 & 0 & 3.682705 & -1.233697 & 0.671120 \\
\hline 15 & 1 & 0 & 1.592778 & -1.300723 & 1.112892 \\
\hline 16 & 6 & 0 & 4.737256 & -0.495432 & 0.120108 \\
\hline 17 & 1 & 0 & 5.297659 & 1.331836 & -0.849935 \\
\hline 18 & 1 & 0 & 3.886452 & -2.191725 & 1.130914 \\
\hline 19 & 6 & 0 & -2.888681 & -2.334099 & -0.102902 \\
\hline 20 & 6 & 0 & -4.190533 & -2.401149 & 0.421259 \\
\hline 21 & 6 & 0 & -2.342257 & -3.504232 & -0.660110 \\
\hline 22 & 6 & 0 & -4.911869 & -3.591909 & 0.401698 \\
\hline 23 & 1 & 0 & -4.635822 & -1.518698 & 0.865249 \\
\hline 24 & 6 & 0 & -3.062544 & -4.692310 & -0.680033 \\
\hline 25 & 1 & 0 & -1.351677 & -3.472961 & -1.098742 \\
\hline 26 & 6 & 0 & -4.352135 & -4.743301 & -0.147936 \\
\hline 27 & 1 & 0 & -5.912657 & -3.620040 & 0.818246 \\
\hline 28 & 1 & 0 & -2.622232 & -5.580048 & -1.120516 \\
\hline 29 & 1 & 0 & -4.914943 & -5.669612 & -0.166982 \\
\hline 30 & 1 & 0 & -3.783522 & 0.463001 & 0.043397 \\
\hline 31 & 6 & 0 & -1.943564 & 2.664441 & 0.002349 \\
\hline 32 & 6 & 0 & -1.336622 & 3.495330 & -0.954485 \\
\hline
\end{tabular}




$\begin{array}{llllll}33 & 6 & 0 & -2.782486 & 3.260144 & 0.958032 \\ 34 & 6 & 0 & -1.559125 & 4.870085 & -0.952299 \\ 35 & 1 & 0 & -0.714489 & 3.054535 & -1.724995 \\ 36 & 6 & 0 & -3.007233 & 4.634087 & 0.957259 \\ 37 & 1 & 0 & -3.245135 & 2.636199 & 1.714387 \\ 38 & 6 & 0 & -2.393936 & 5.446009 & 0.004048 \\ 39 & 1 & 0 & -1.088469 & 5.491022 & -1.706696 \\ 40 & 1 & -3.655792 & 5.072566 & 1.707759 \\ 41 & 1 & 0 & -2.566554 & 6.516276 & 0.005191 \\ 42 & 8 & 0 & 7.082099 & -0.373255 & -0.339701 \\ 43 & 6 & 0 & 6.143930 & -0.984974 & 0.119188 \\ 44 & 8 & 0 & 6.267650 & -2.202705 & 0.690973 \\ 45 & 6 & 0 & 7.599570 & -2.746118 & 0.728291 \\ 46 & 1 & 0 & 7.504070 & -3.713229 & 1.217021 \\ 47 & 1 & 0.961756 & -2.862733 & -0.282893 \\ 48 & 1 & 0 & -2.091624 & 1.295959\end{array}$

Total electronic energy (B3LYP/6-311+G $(\mathrm{d}, \mathrm{p}))=-1153.54426$ hartrees 
Cartesian coordinates for 1,3-diphenyl-6-4-(trifluoromethyl)phenyl]fulvene (20).

\begin{tabular}{|c|c|c|c|c|c|}
\hline \multirow{2}{*}{$\begin{array}{l}\text { Center } \\
\text { Number }\end{array}$} & \multirow{2}{*}{$\begin{array}{l}\text { Atomic } \\
\text { Number }\end{array}$} & \multirow{2}{*}{$\begin{array}{l}\text { Atomic } \\
\text { Type }\end{array}$} & \multicolumn{3}{|c|}{ Coordinates (Angstroms) } \\
\hline & & & $\mathrm{X}$ & Y & Z \\
\hline 1 & 6 & 0 & -1.055197 & -0.940201 & -0.121531 \\
\hline 2 & 6 & 0 & -0.819449 & 0.498213 & -0.067400 \\
\hline 3 & 6 & 0 & -2.165247 & 1.137863 & -0.017528 \\
\hline 4 & 6 & 0 & -3.079859 & 0.136503 & 0.024430 \\
\hline 5 & 6 & 0 & -2.400348 & -1.168735 & -0.062014 \\
\hline 6 & 1 & 0 & -0.286350 & -1.691715 & -0.197503 \\
\hline 7 & 6 & 0 & 0.347737 & 1.187433 & -0.001479 \\
\hline 8 & 1 & 0 & 0.272180 & 2.271127 & -0.008827 \\
\hline 9 & 6 & 0 & 1.719156 & 0.679765 & 0.057163 \\
\hline 10 & 6 & 0 & 2.750514 & 1.476938 & -0.472961 \\
\hline 11 & 6 & 0 & 2.073966 & -0.542135 & 0.656334 \\
\hline 12 & 6 & 0 & 4.071733 & 1.052369 & -0.457935 \\
\hline 13 & 1 & 0 & 2.504949 & 2.437355 & -0.912520 \\
\hline
\end{tabular}




\begin{tabular}{|c|c|c|c|c|c|}
\hline 14 & 6 & 0 & 3.395749 & -0.966975 & 0.683426 \\
\hline 15 & 1 & 0 & 1.316053 & -1.142519 & 1.140359 \\
\hline 16 & 6 & 0 & 4.397569 & -0.176125 & 0.118651 \\
\hline 17 & 1 & 0 & 4.848800 & 1.677466 & -0.879652 \\
\hline 18 & 1 & 0 & 3.652439 & -1.903985 & 1.161681 \\
\hline 19 & 6 & 0 & -3.087832 & -2.464419 & -0.096135 \\
\hline 20 & 6 & 0 & -4.385883 & -2.605743 & 0.422577 \\
\hline 21 & 6 & 0 & -2.468900 & -3.603265 & -0.642198 \\
\hline 22 & 6 & 0 & -5.034132 & -3.837835 & 0.408849 \\
\hline 23 & 1 & 0 & -4.885611 & -1.748494 & 0.857764 \\
\hline 24 & 6 & 0 & -3.116317 & -4.832545 & -0.656358 \\
\hline 25 & 1 & 0 & -1.479948 & -3.515797 & -1.076828 \\
\hline 26 & 6 & 0 & -4.403189 & -4.957134 & -0.129700 \\
\hline 27 & 1 & 0 & -6.033393 & -3.923208 & 0.821112 \\
\hline 28 & 1 & 0 & -2.621295 & -5.695414 & -1.087963 \\
\hline 29 & 1 & 0 & -4.908950 & -5.915830 & -0.144466 \\
\hline 30 & 1 & 0 & -4.152261 & 0.272862 & 0.040892 \\
\hline 31 & 6 & 0 & -2.449632 & 2.583654 & -0.005948 \\
\hline 32 & 6 & 0 & -1.902084 & 3.446132 & -0.970456 \\
\hline
\end{tabular}




$\begin{array}{llllll}33 & 6 & 0 & -3.313043 & 3.130811 & 0.956745 \\ 34 & 6 & 0 & -2.205783 & 4.805313 & -0.969023 \\ 35 & 1 & 0 & -1.262360 & 3.040596 & -1.746002 \\ 36 & 6 & 0 & -3.618621 & 4.489095 & 0.955454 \\ 37 & 1 & 0 & -3.730928 & 2.482608 & 1.718682 \\ 38 & 6 & 0 & -3.063835 & 5.333207 & -0.005483 \\ 39 & 1 & -1.780407 & 5.451045 & -1.729446 \\ 40 & 1 & 0 & -4.284829 & 4.890415 & 1.711219 \\ 41 & 1 & 0 & -3.299681 & 6.391318 & -0.005011 \\ 42 & 6 & 0 & 5.819314 & -0.664211 & 0.095164 \\ 43 & 9 & 0 & 6.087566 & -1.376824 & -1.030284 \\ 44 & 9 & 0 & 6.101381 & -1.475847 & 1.139090 \\ 45 & 9 & 0 & 6.711121 & 0.351845 & 0.126515\end{array}$

Total electronic energy (B $3 L Y P / 6-311+G(d, p))=-1262.74649$ hartrees 
Cartesian coordinates for 1,3-diphenyl-6-(4-trimethylammoniu)phenyl)fulvene (21).

\begin{tabular}{|c|c|c|c|c|c|}
\hline \multirow{2}{*}{$\begin{array}{l}\text { Center } \\
\text { Number }\end{array}$} & \multirow{2}{*}{$\begin{array}{l}\text { Atomic } \\
\text { Number }\end{array}$} & \multirow{2}{*}{$\begin{array}{l}\text { Atomic } \\
\text { Type }\end{array}$} & \multicolumn{3}{|c|}{ Coordinates (Angstroms) } \\
\hline & & & $\mathrm{X}$ & Y & Z \\
\hline 1 & 6 & 0 & 1.102982 & 0.947117 & -0.107882 \\
\hline 2 & 6 & 0 & 0.861351 & -0.489198 & -0.070218 \\
\hline 3 & 6 & 0 & 2.208239 & -1.140972 & -0.007661 \\
\hline 4 & 6 & 0 & 3.124417 & -0.147298 & 0.039510 \\
\hline 5 & 6 & 0 & 2.449951 & 1.167859 & -0.037596 \\
\hline 6 & 1 & 0 & 0.349659 & 1.704112 & -0.254962 \\
\hline 7 & 6 & 0 & -0.301779 & -1.188740 & -0.045635 \\
\hline 8 & 1 & 0 & -0.221301 & -2.271073 & -0.071929 \\
\hline 9 & 6 & 0 & -1.671973 & -0.686103 & -0.010582 \\
\hline 10 & 6 & 0 & -2.704564 & -1.494732 & -0.515886 \\
\hline 11 & 6 & 0 & -2.043390 & 0.552765 & 0.547807 \\
\hline 12 & 6 & 0 & -4.033032 & -1.082203 & -0.517191 \\
\hline 13 & 1 & 0 & -2.462667 & -2.466532 & -0.930164 \\
\hline
\end{tabular}




\begin{tabular}{|c|c|c|c|c|c|}
\hline 14 & 6 & 0 & -3.364597 & 0.973729 & 0.558294 \\
\hline 15 & 1 & 0 & -1.293774 & 1.178323 & 1.011296 \\
\hline 16 & 6 & 0 & -4.361072 & 0.160886 & 0.015272 \\
\hline 17 & 1 & 0 & -4.773886 & -1.747879 & -0.934966 \\
\hline 18 & 1 & 0 & -3.594530 & 1.932004 & 1.007424 \\
\hline 19 & 6 & 0 & 3.146541 & 2.454986 & -0.061376 \\
\hline 20 & 6 & 0 & 4.509003 & 2.532128 & -0.398781 \\
\hline 21 & 6 & 0 & 2.472330 & 3.651830 & 0.244262 \\
\hline 22 & 6 & 0 & 5.165969 & 3.758081 & -0.445958 \\
\hline 23 & 1 & 0 & 5.056119 & 1.630936 & -0.646558 \\
\hline 24 & 6 & 0 & 3.129475 & 4.873975 & 0.201469 \\
\hline 25 & 1 & 0 & 1.430263 & 3.617515 & 0.540129 \\
\hline 26 & 6 & 0 & 4.480537 & 4.933222 & -0.146473 \\
\hline 27 & 1 & 0 & 6.214754 & 3.794884 & -0.716822 \\
\hline 28 & 1 & 0 & 2.593842 & 5.783475 & 0.448806 \\
\hline 29 & 1 & 0 & 4.994071 & 5.887096 & -0.176618 \\
\hline 30 & 1 & 0 & 4.193219 & -0.289047 & 0.109834 \\
\hline 31 & 6 & 0 & 2.473063 & -2.590190 & 0.046890 \\
\hline 32 & 6 & 0 & 1.966397 & -3.461879 & -0.931382 \\
\hline
\end{tabular}




\begin{tabular}{|c|c|c|c|c|c|}
\hline 33 & 6 & 0 & 3.277963 & -3.126220 & 1.064118 \\
\hline 34 & 6 & 0 & 2.254390 & -4.823829 & -0.890390 \\
\hline 35 & 1 & 0 & 1.379517 & -3.062958 & -1.751389 \\
\hline 36 & 6 & 0 & 3.565401 & -4.487955 & 1.103220 \\
\hline 37 & 1 & 0 & 3.666824 & -2.468664 & 1.833286 \\
\hline 38 & 6 & 0 & 3.052444 & -5.342176 & 0.128287 \\
\hline 39 & 1 & 0 & 1.868484 & -5.478631 & -1.663680 \\
\hline 40 & 1 & 0 & 4.187589 & -4.882866 & 1.898313 \\
\hline 41 & 1 & 0 & 3.278116 & -6.401811 & 0.158367 \\
\hline 42 & 7 & 0 & -5.787657 & 0.661235 & 0.036984 \\
\hline 43 & 6 & 0 & -6.754931 & -0.310096 & -0.591123 \\
\hline 44 & 1 & 0 & -7.748614 & 0.130332 & -0.539194 \\
\hline 45 & 1 & 0 & -6.479436 & -0.473933 & -1.630085 \\
\hline 46 & 1 & 0 & -6.739611 & -1.245730 & -0.037591 \\
\hline 47 & 6 & 0 & -6.231725 & 0.887001 & 1.468010 \\
\hline 48 & 1 & 0 & -6.151262 & -0.056275 & 2.004091 \\
\hline 49 & 1 & 0 & -5.589984 & 1.630254 & 1.931828 \\
\hline 50 & 1 & 0 & -7.263201 & 1.237094 & 1.460624 \\
\hline 51 & 6 & 0 & -5.883805 & 1.962238 & -0.733673 \\
\hline
\end{tabular}




$\begin{array}{llllll}52 & 1 & 0 & -5.241979 & 2.704667 & -0.268737 \\ 53 & 1 & 0 & -6.919054 & 2.300856 & -0.716892 \\ 54 & 1 & 0 & -5.557071 & 1.779842 & -1.755293\end{array}$

Total electronic energy (B3LYP/6-311+G(d,p)) = -1099.28593 hartrees 


\section{Cyclic Voltammograms for}

1,3-diphenyl-6-arylfulvenes 5 - 21 
1,3-diphenyl-6-[4-(dimethylamino)phenyl]fulvene (5)

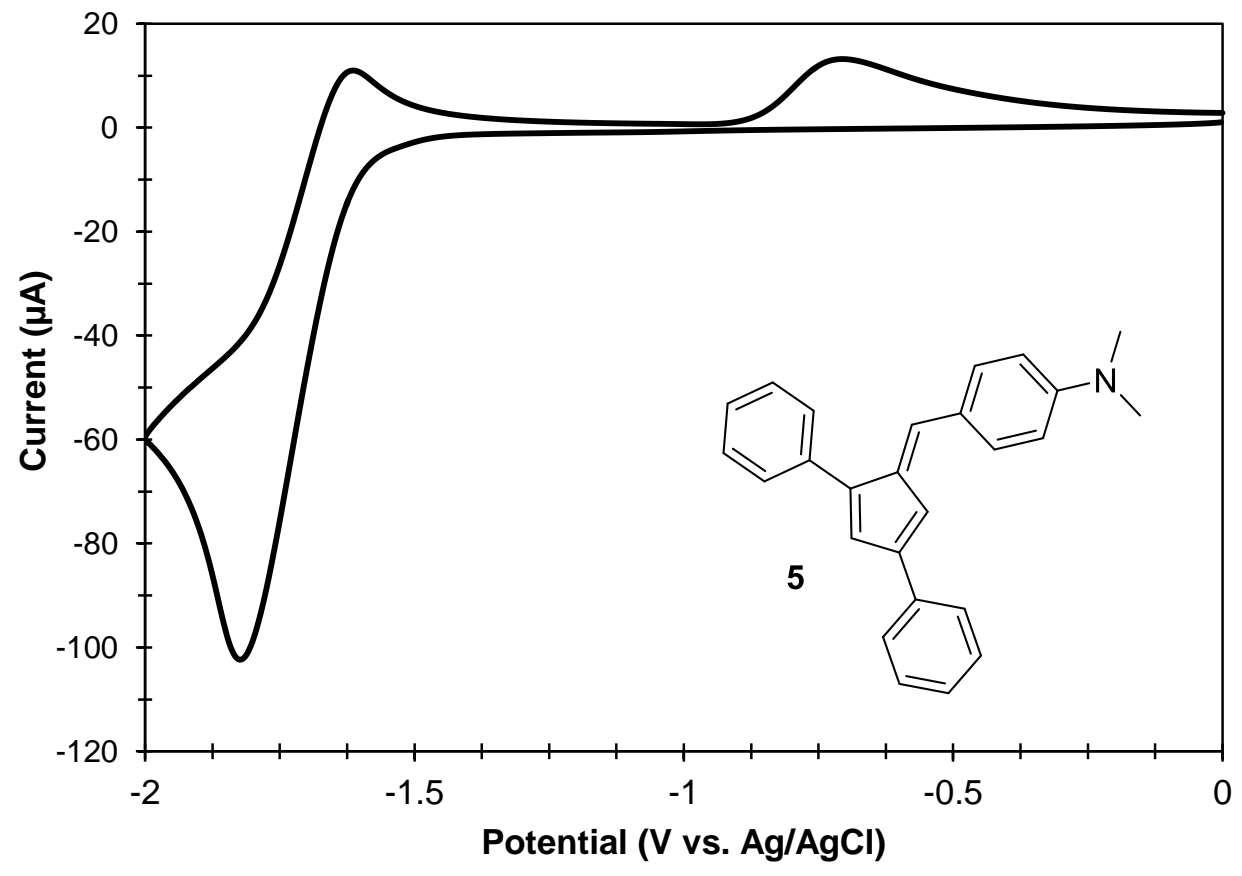


1,3-diphenyl-6-(4-methoxyphenyl)fulvene (6)

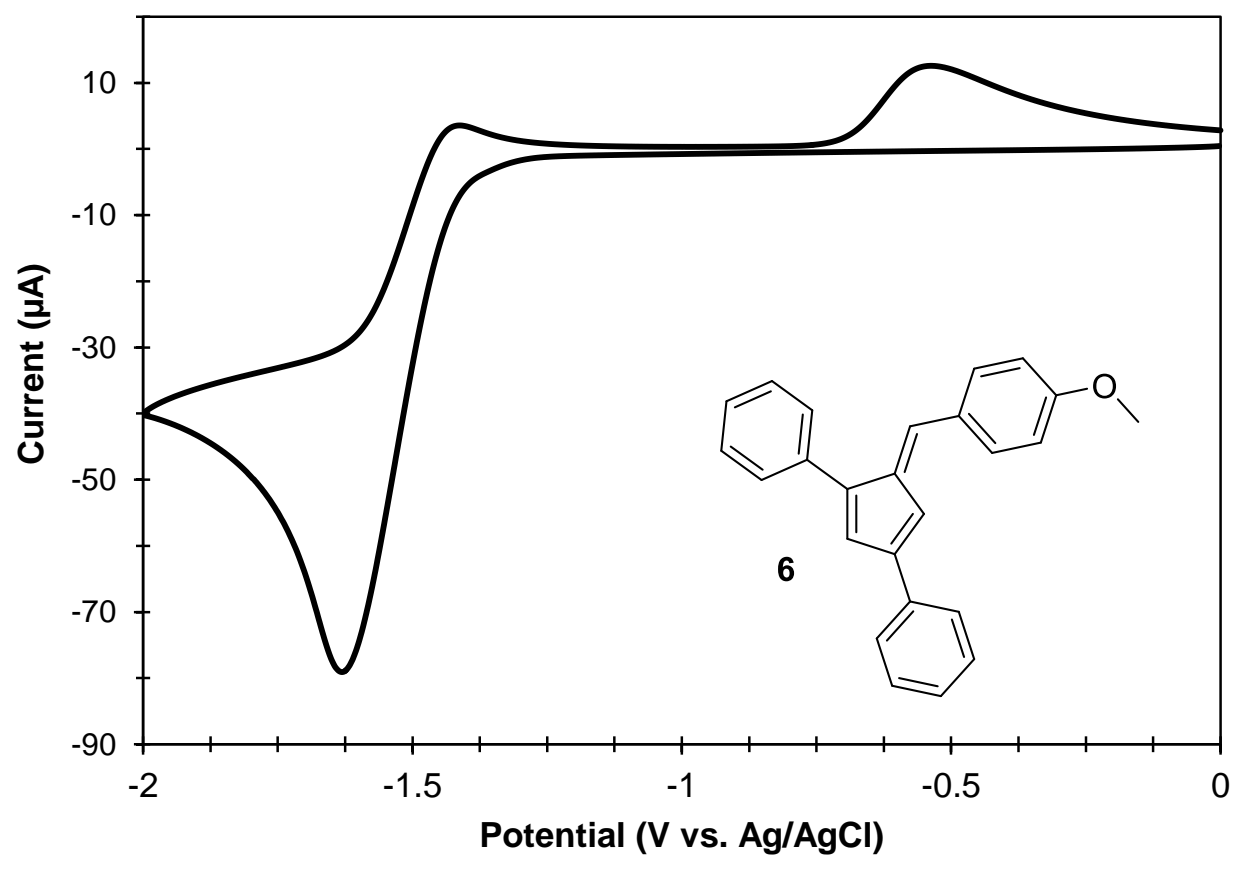


1,3-diphenyl-6-(4-methylphenyl)fulvene (7)

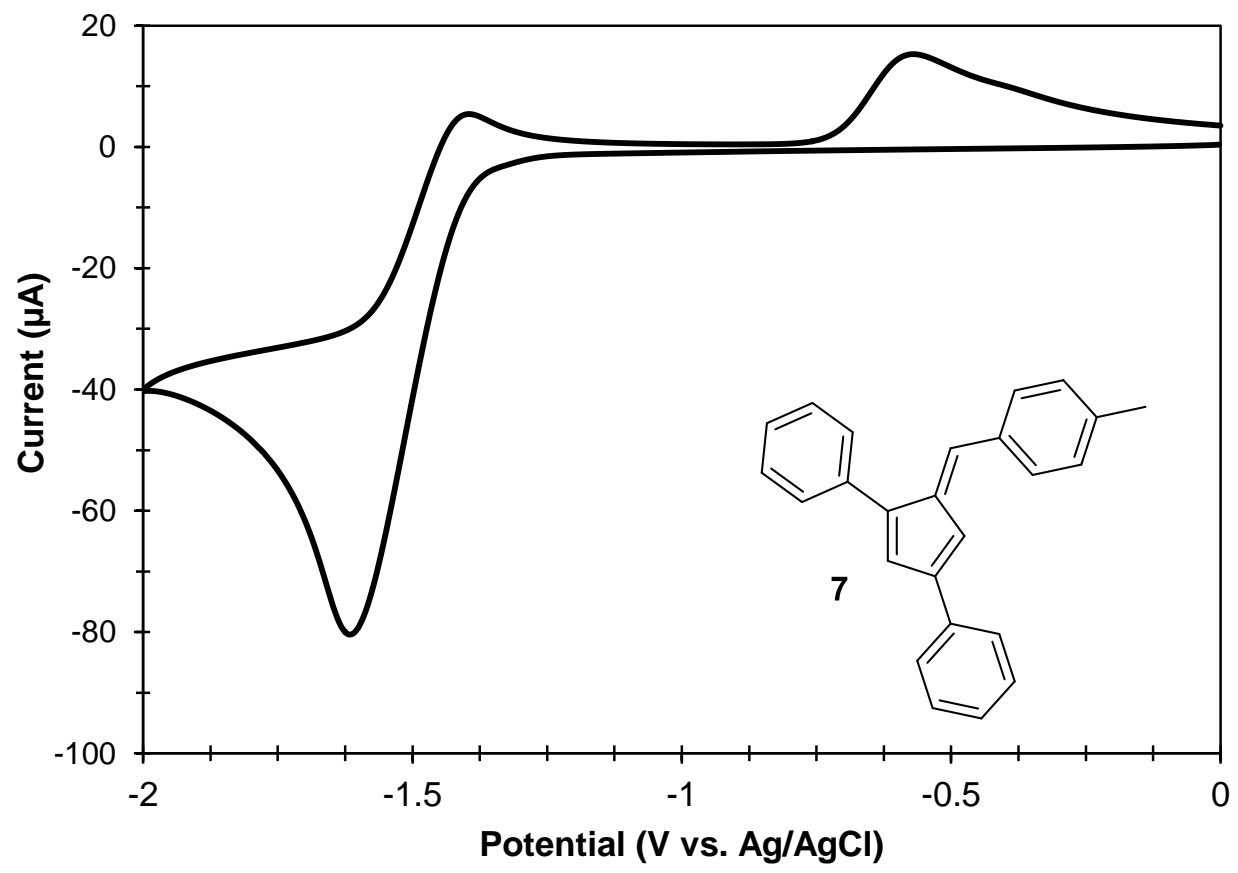


1,3-diphenyl-6-(3-phenoxyphenyl)fulvene (8)

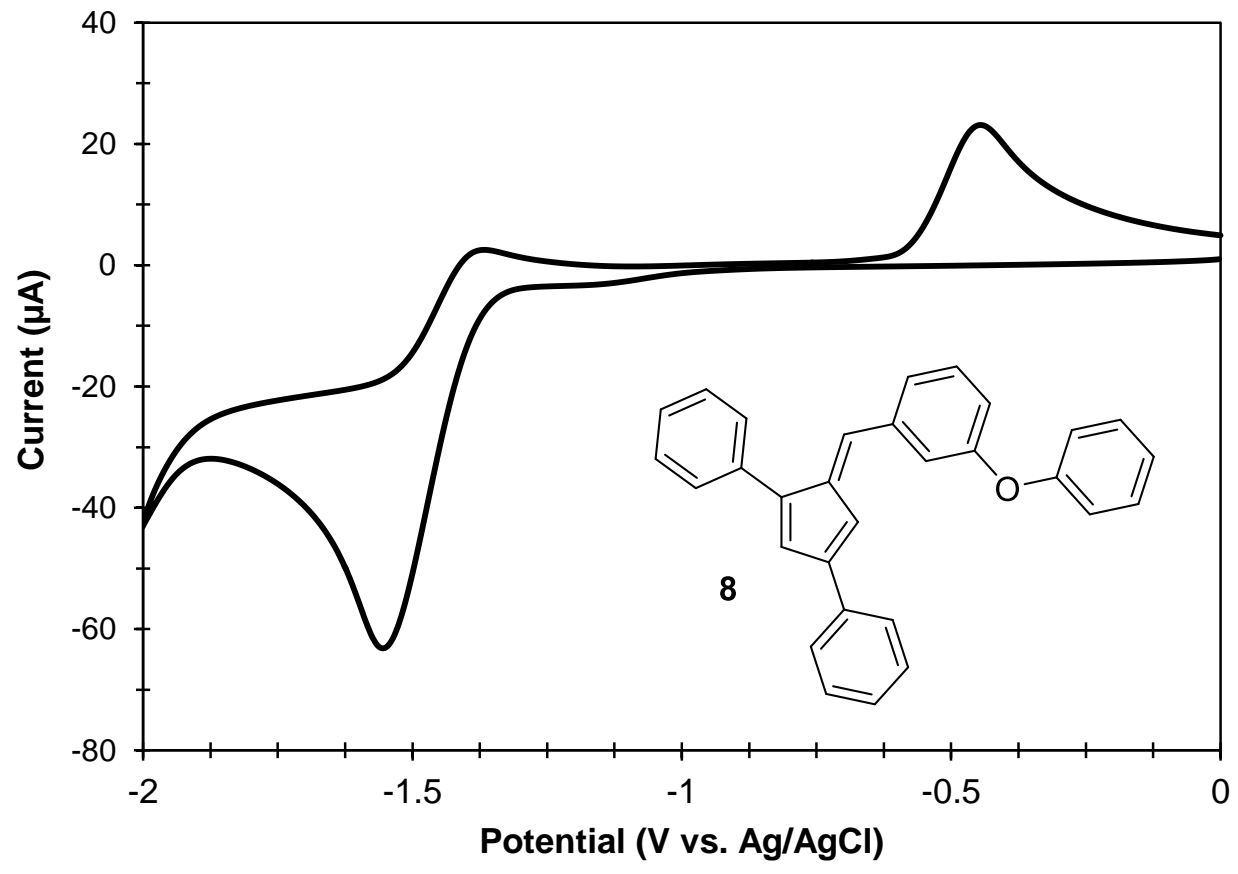


1,3-diphenyl-6-(4-biphenyl)fulvene (9)

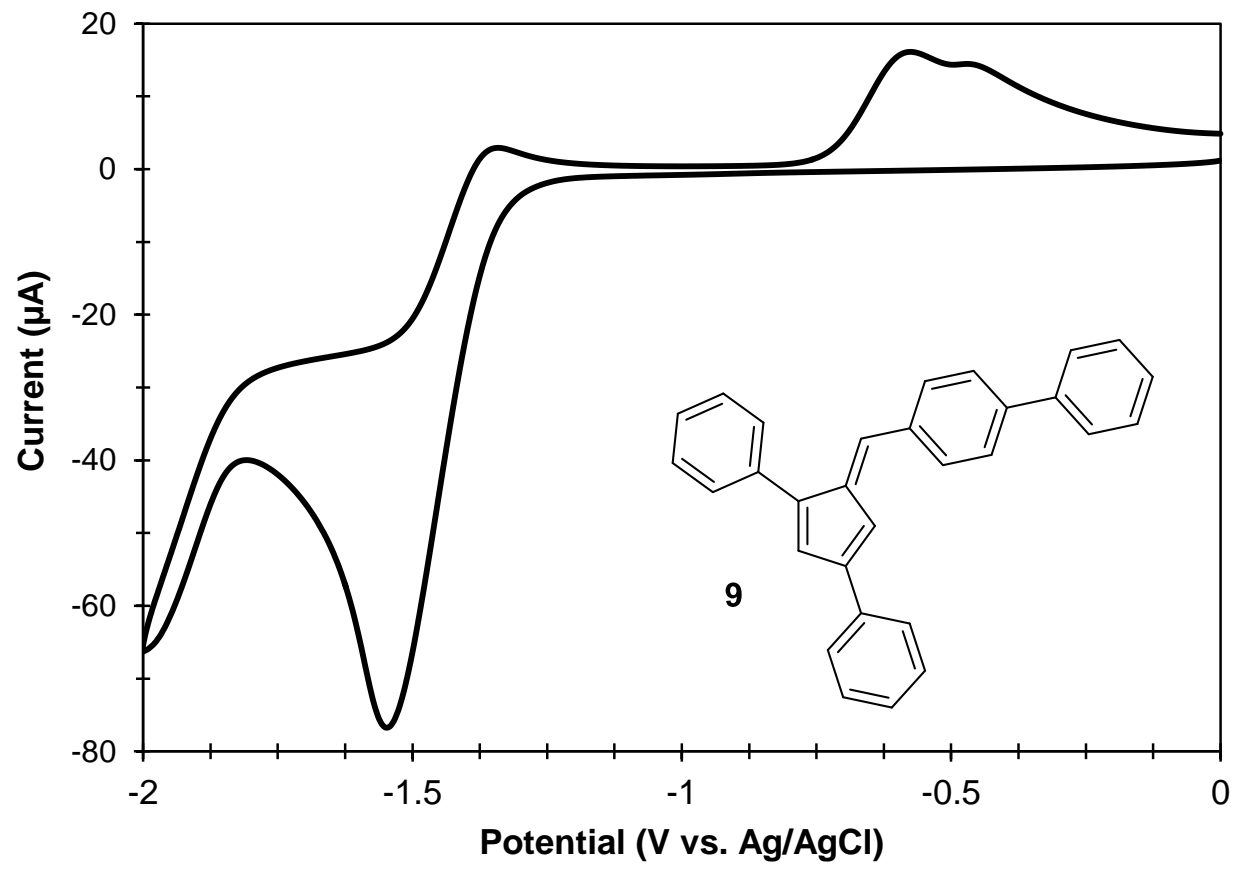


1,3-diphenyl-6-(3-methylphenyl)fulvene (10)

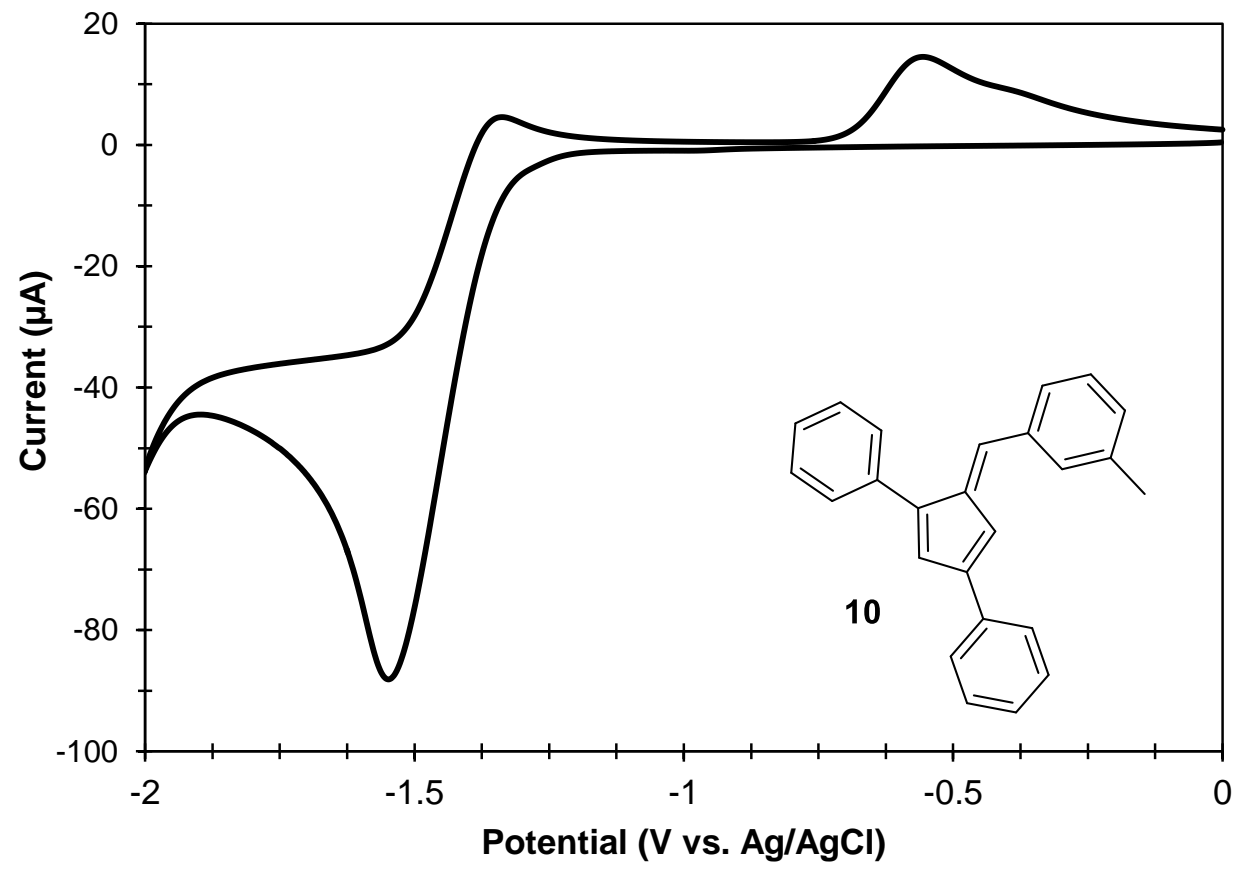


1,3-diphenyl-6-(4-fluorophenyl)fulvene (11)

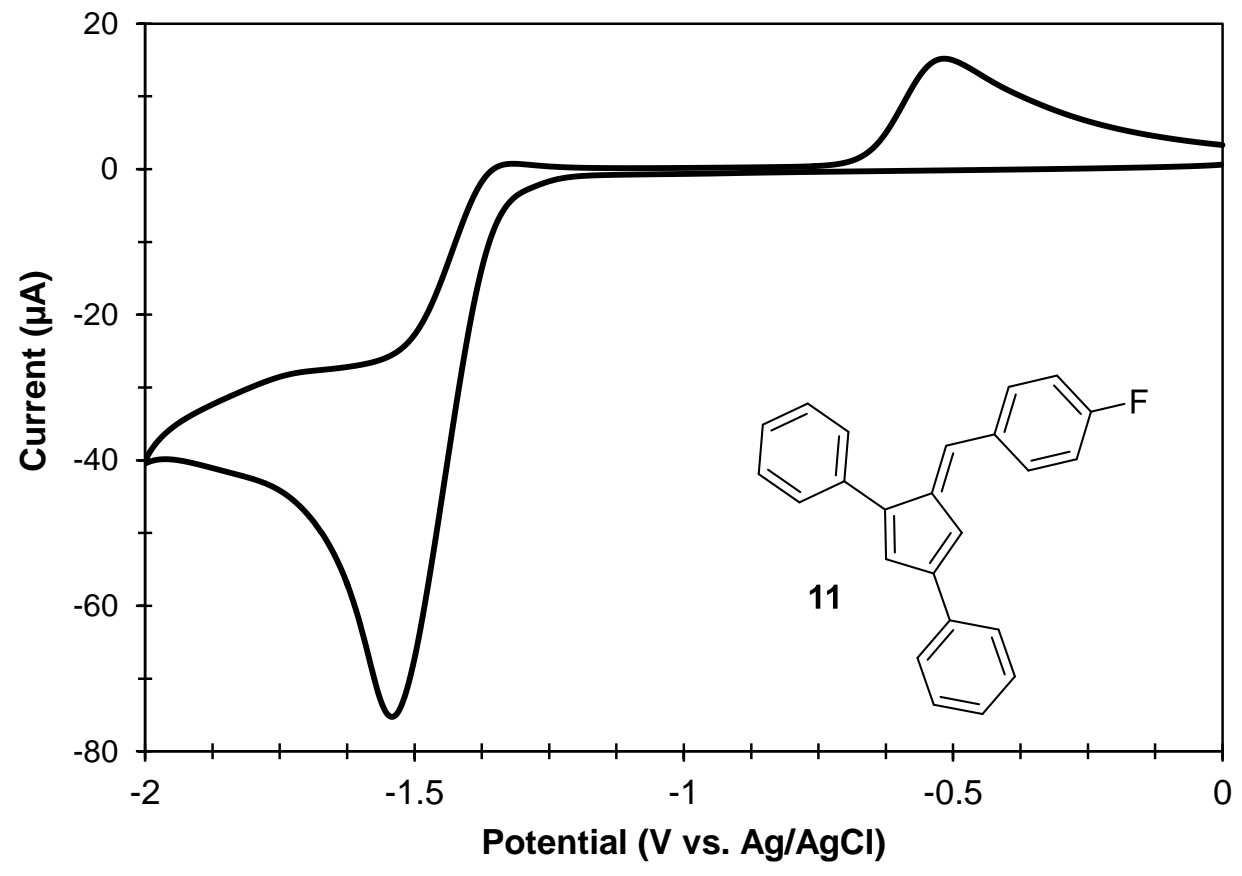


1,3-diphenyl-6-(3-methoxyphenyl)fulvene (12)

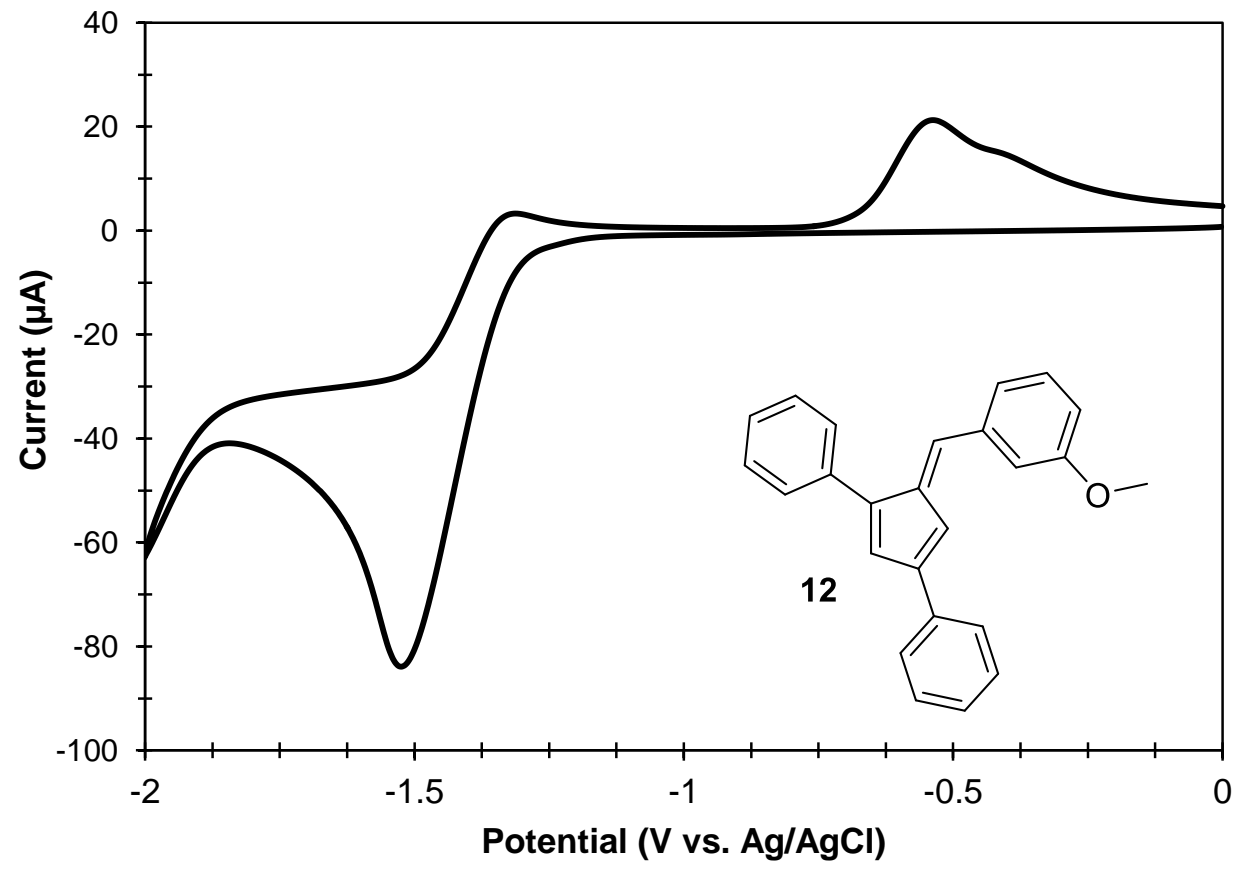


1,3-diphenyl-6-(3-vinylphenyl)fulvene (13)

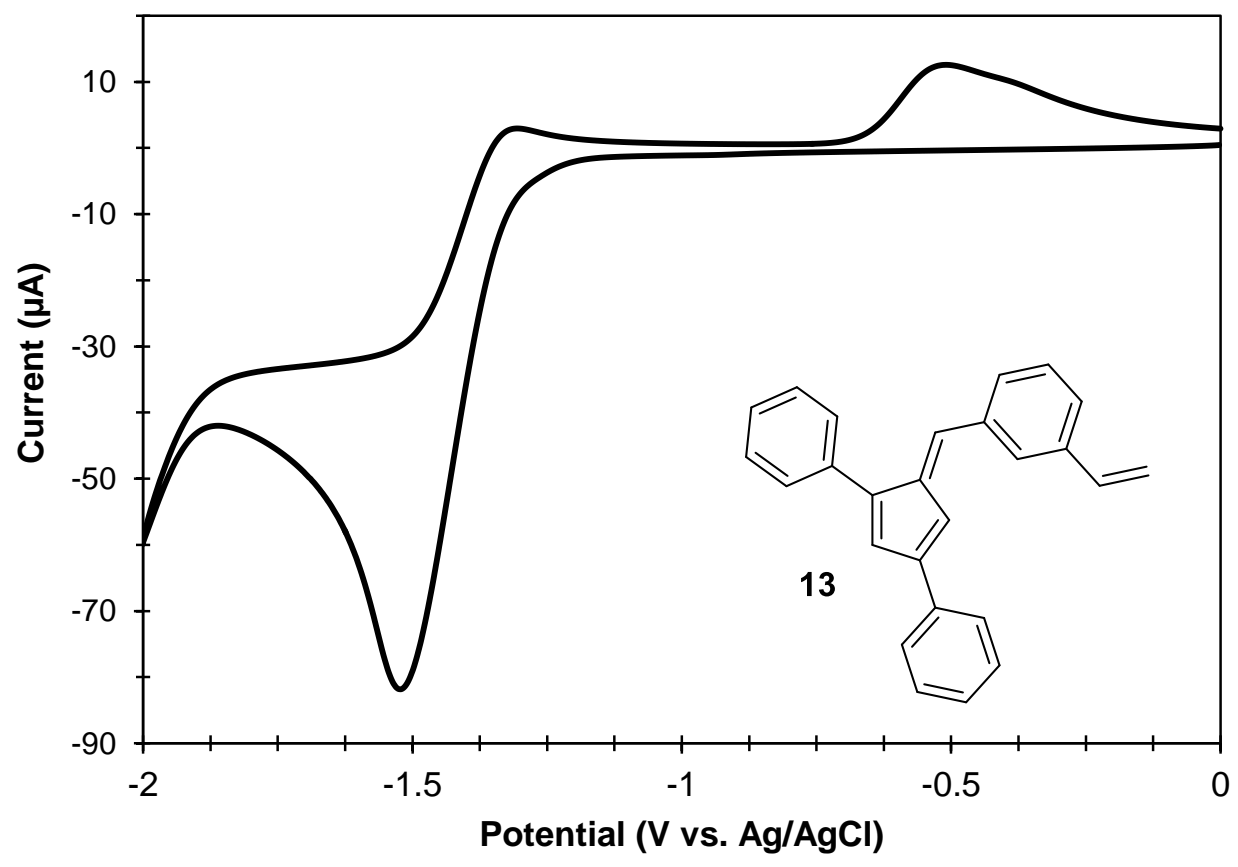


1,3-diphenyl-6-[4-(2-pyridyl)phenyl]fulvene (14)

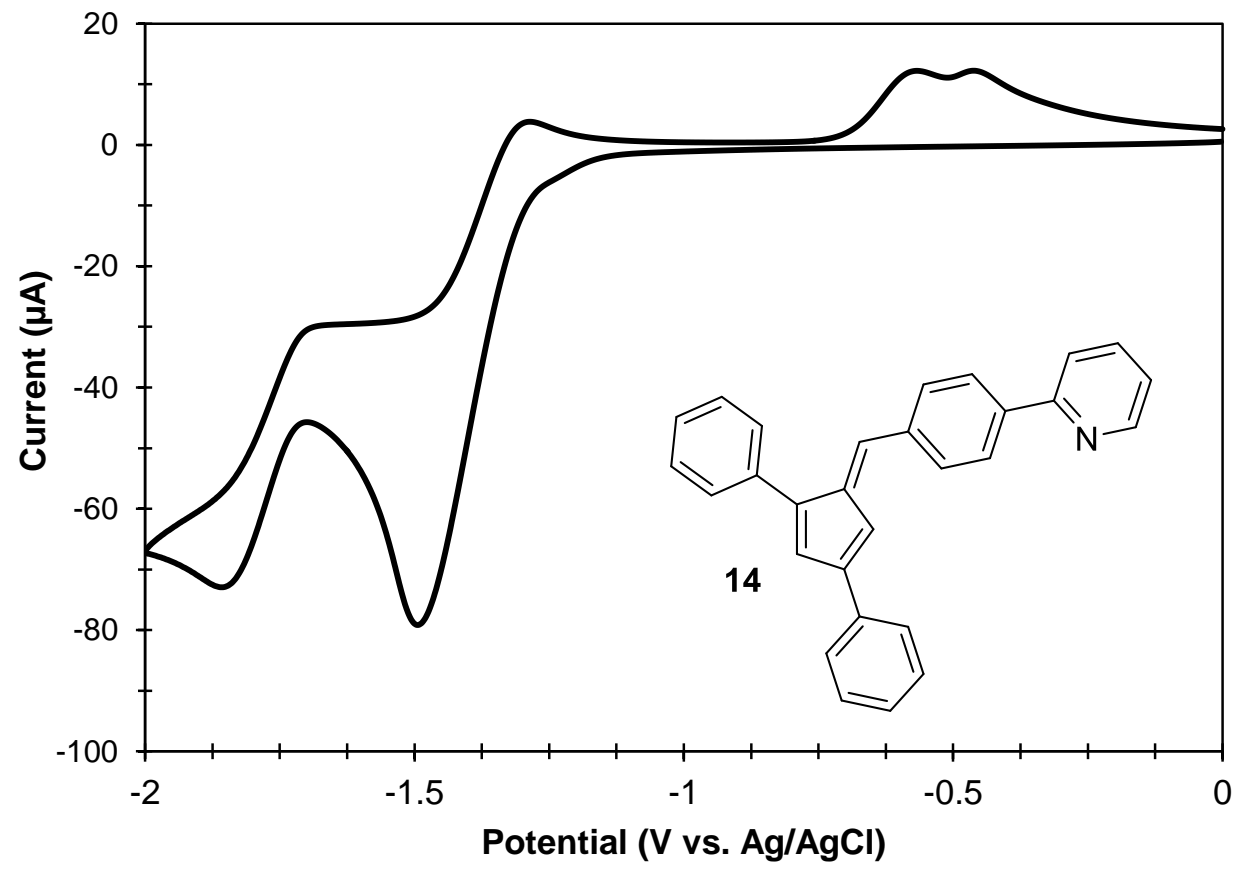


1,3-diphenyl-6-(4-bromophenyl)fulvene (15)

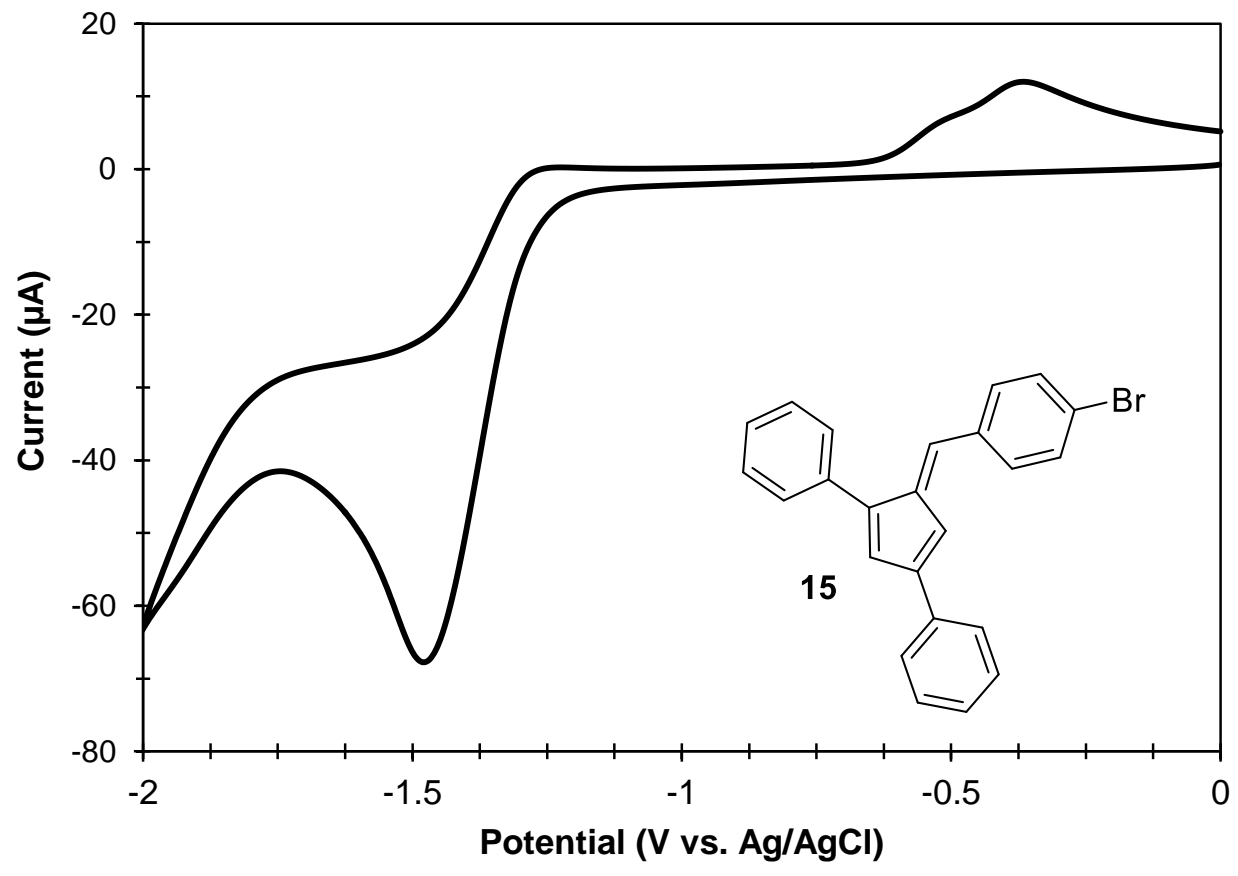


1,3-diphenyl-6-(4-trifluoromethoxyphenyl)fulvene (16)

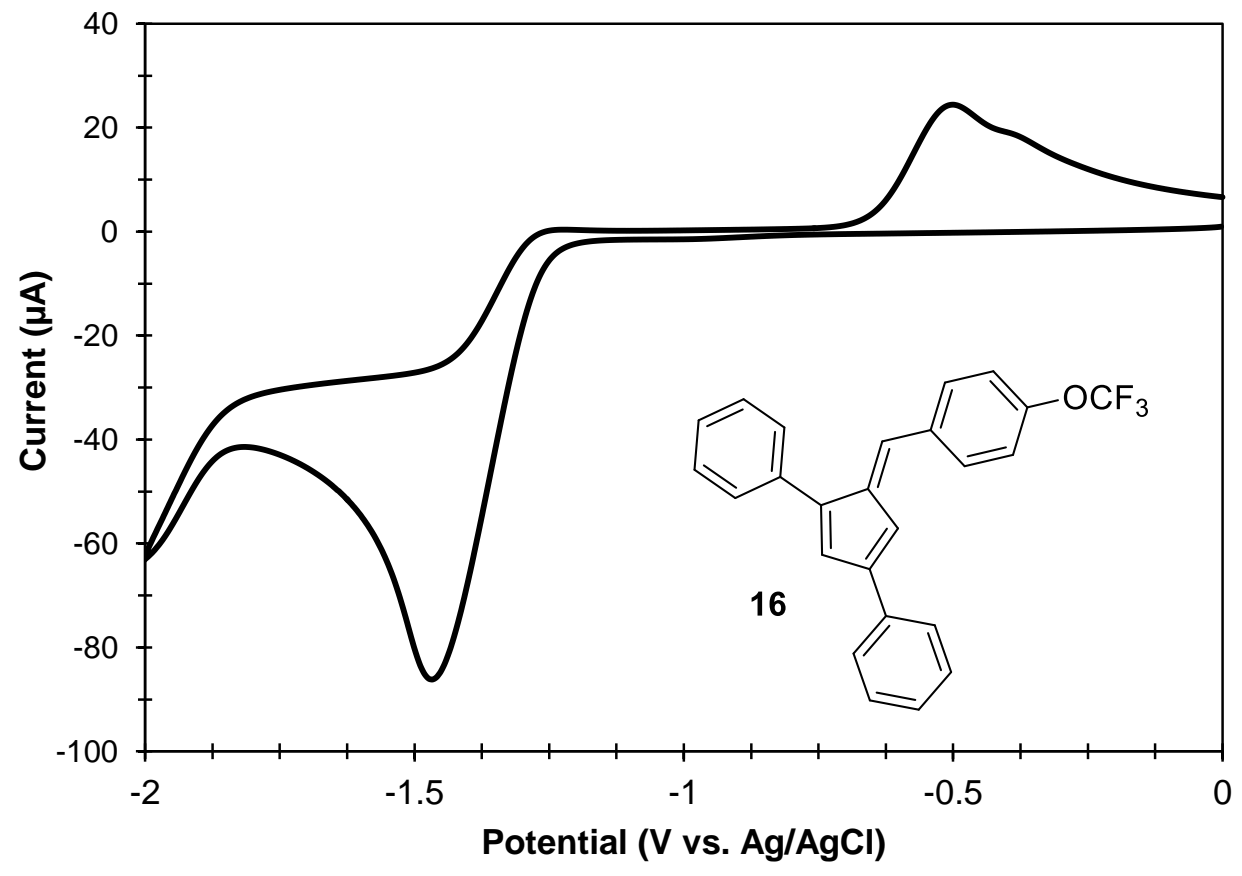


1,3-diphenyl-6-(4-ethynylphenyl)fulvene (17)

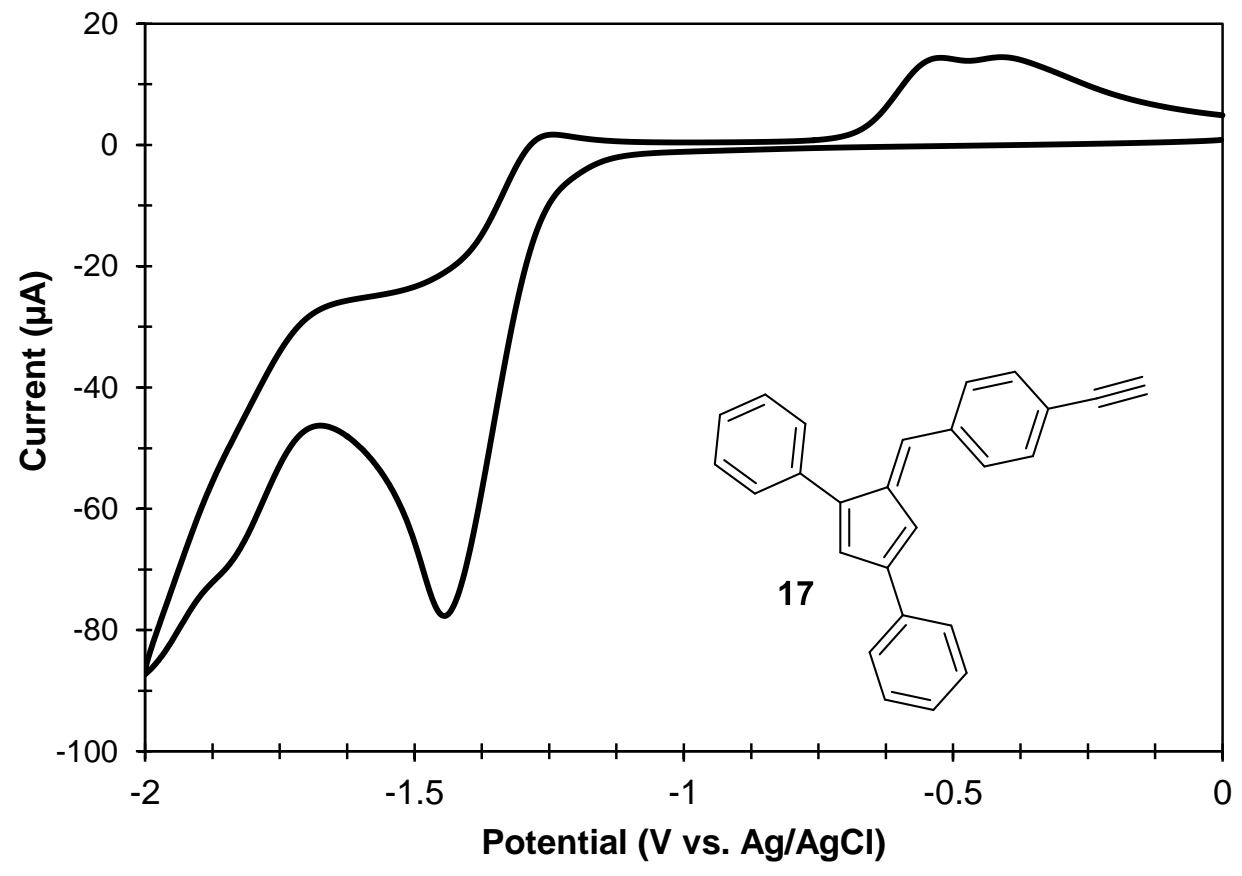


1,3-diphenyl-6-[4-(4-pyridyl)phenyl]fulvene (18)

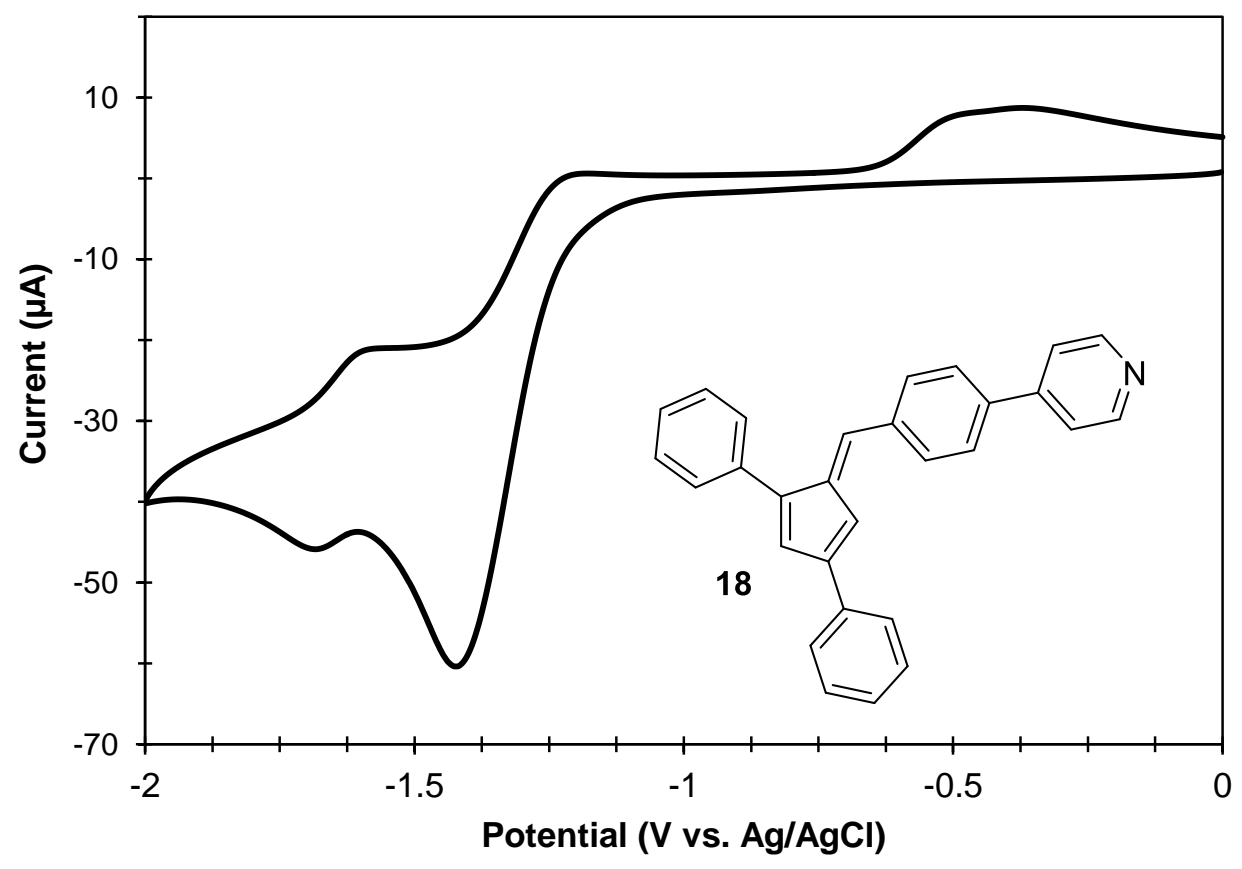


1,3-diphenyl-6-[4-(methyl benzoate)phenylfulvene (19)

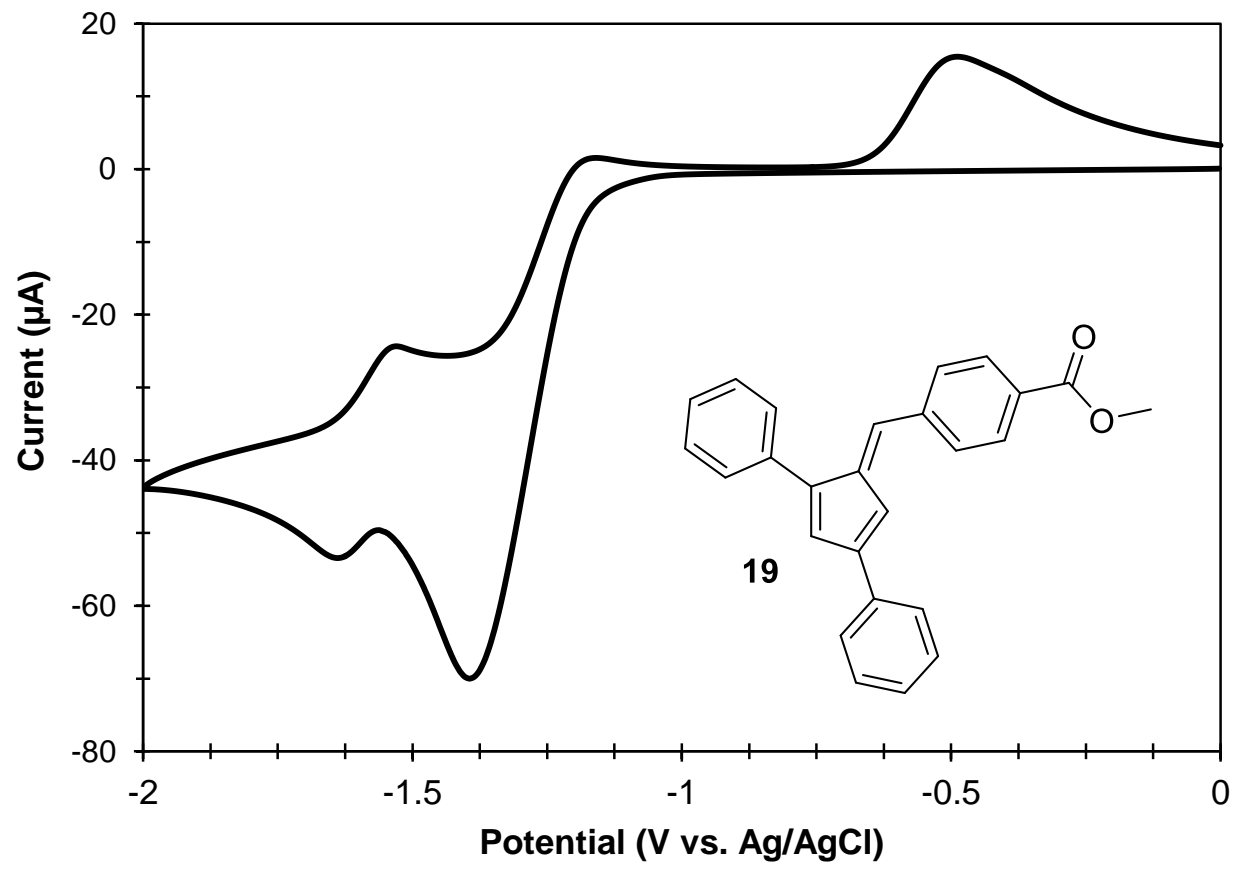


1,3-diphenyl-6-(4-trifluoromethylphenyl)fulvene (20)

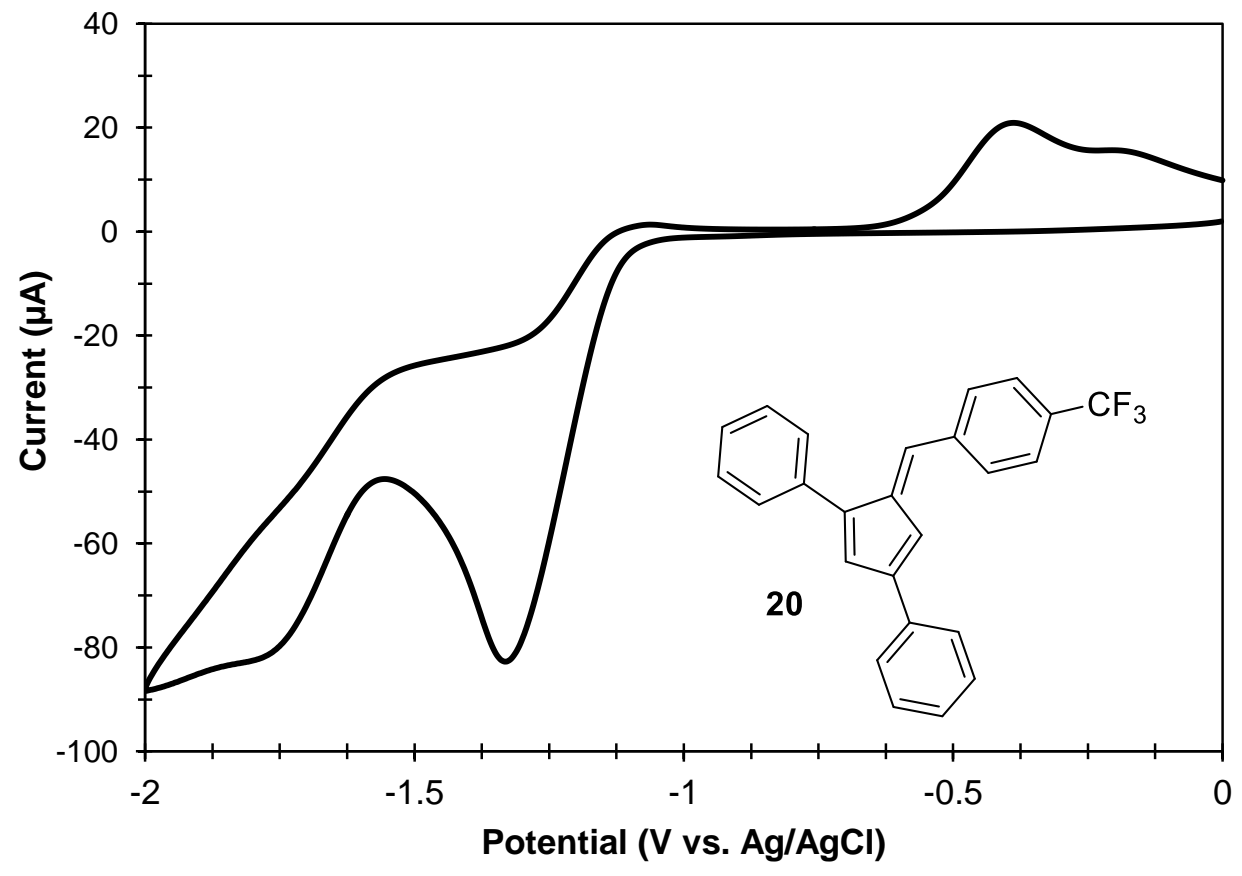


1,3-diphenyl-6-[4-(trimethylammonium iodide)phenyl]fulvene (21)

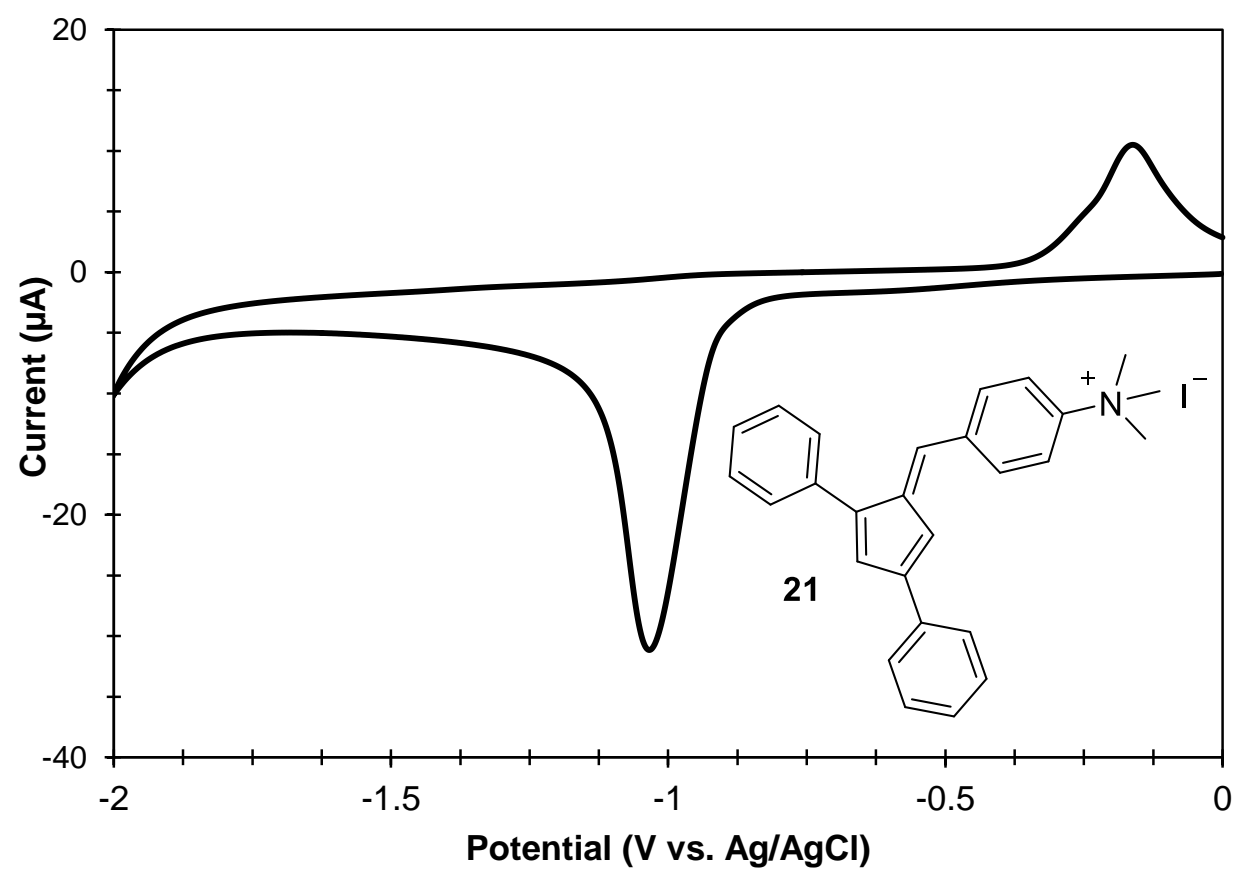


NMR Spectra for

Compounds $4-23$ 
1,3-diphenyl-5-benzylcyclopentadiene (4)

${ }^{1} \mathrm{H}-\mathrm{NMR}$ (400 MHz, $\mathrm{CDCl}_{3}$ )

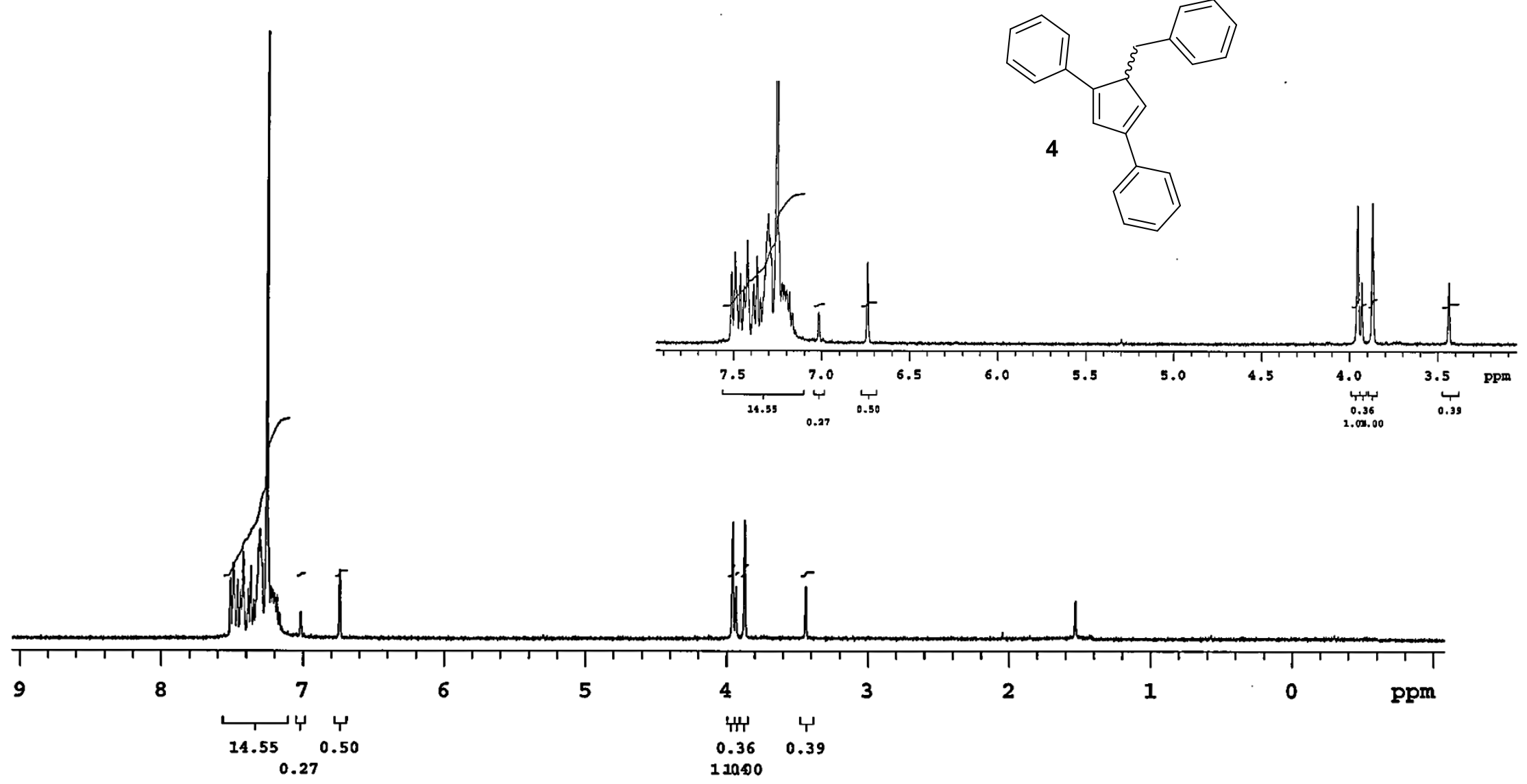


1,3-diphenyl-5-benzylcyclopentadiene (4)

${ }^{13} \mathrm{C}$-NMR (100 MHz, $\left.\mathrm{CDCl}_{3}\right)$

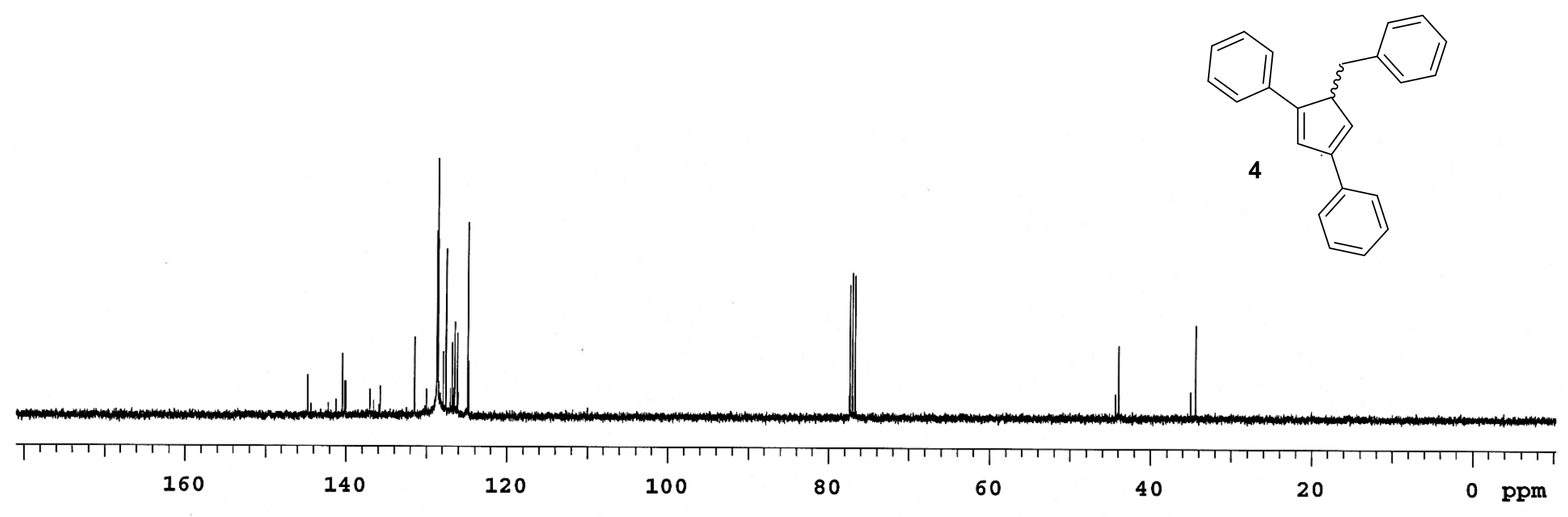


1,3-diphenyl-6-[4-(dimethylamino)phenyl]fulvene (5)

${ }^{1} \mathrm{H}-\mathrm{NMR}$ (400 MHz, $\mathrm{CDCl}_{3}$ )

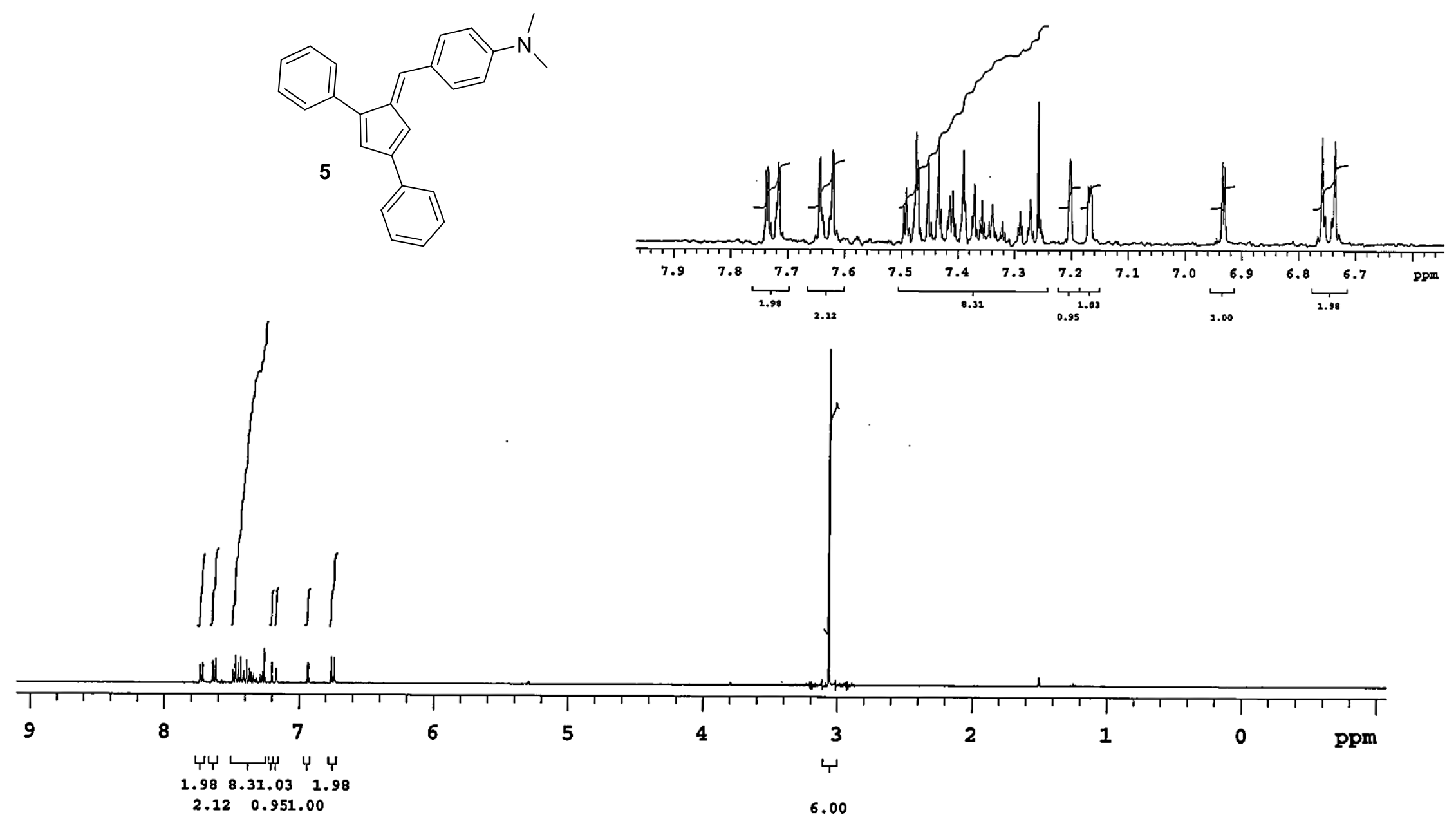


1,3-diphenyl-6-[4-(dimethylamino)phenyl]fulvene (5)

${ }^{13} \mathrm{C}-\mathrm{NMR}\left(100 \mathrm{MHz}, \mathrm{CDCl}_{3}\right)$

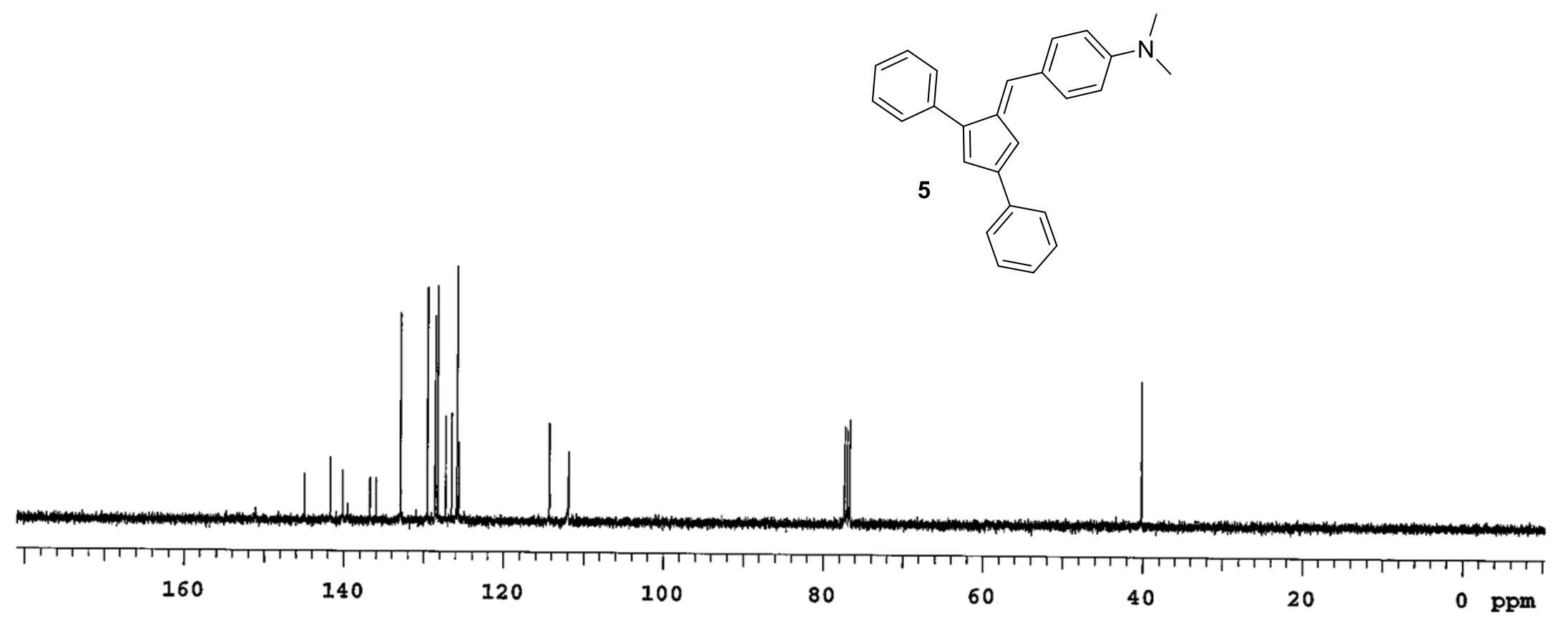


1,3-diphenyl-6-(4-methoxyphenyl)fulvene (6)

${ }^{1} \mathrm{H}-\mathrm{NMR}$ (400 MHz, $\mathrm{CDCl}_{3}$ )

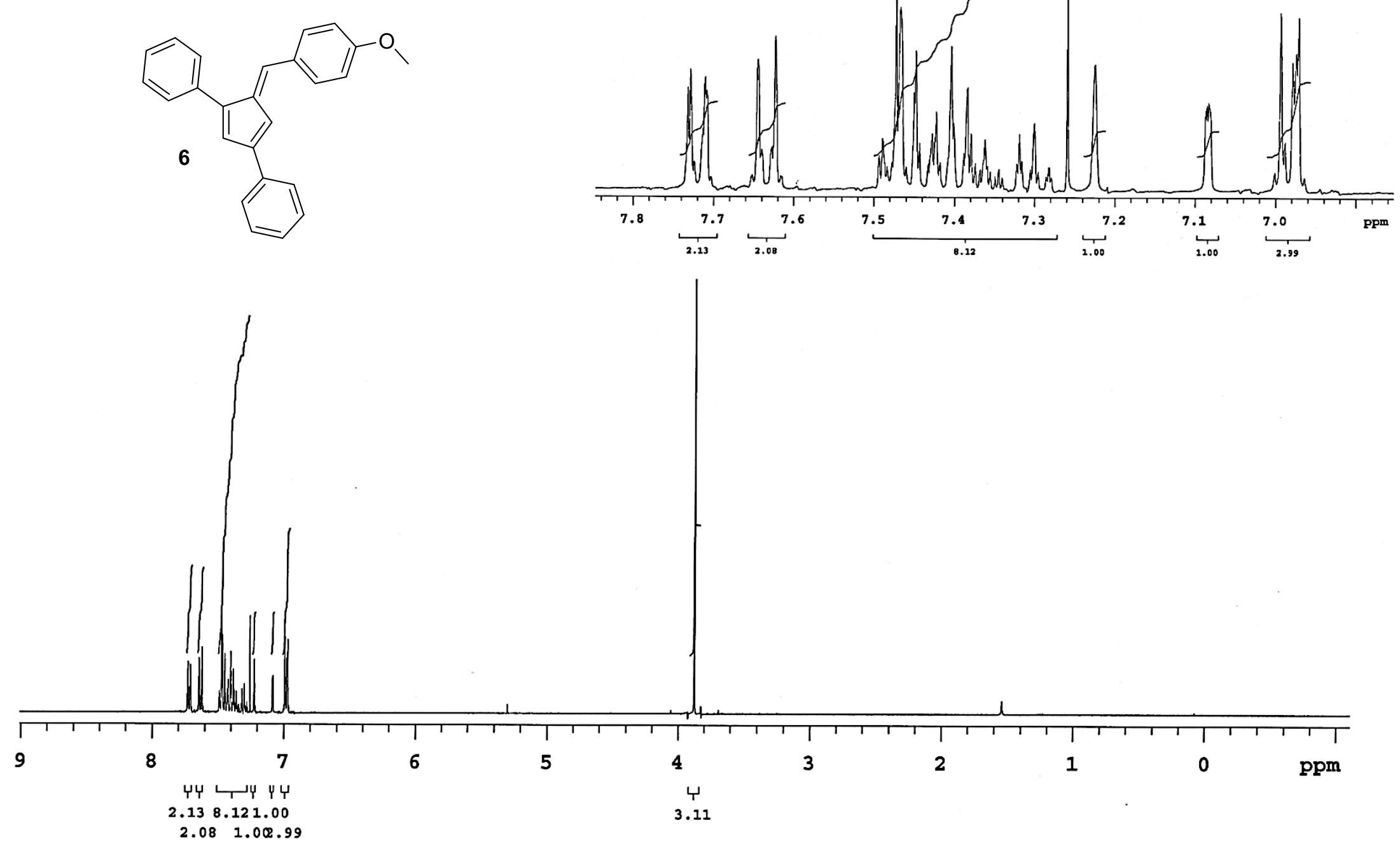


1,3-diphenyl-6-(4-methoxyphenyl)fulvene (6)

${ }^{13} \mathrm{C}$-NMR (400 $\mathrm{MHz}, \mathrm{CDCl}_{3}$ )

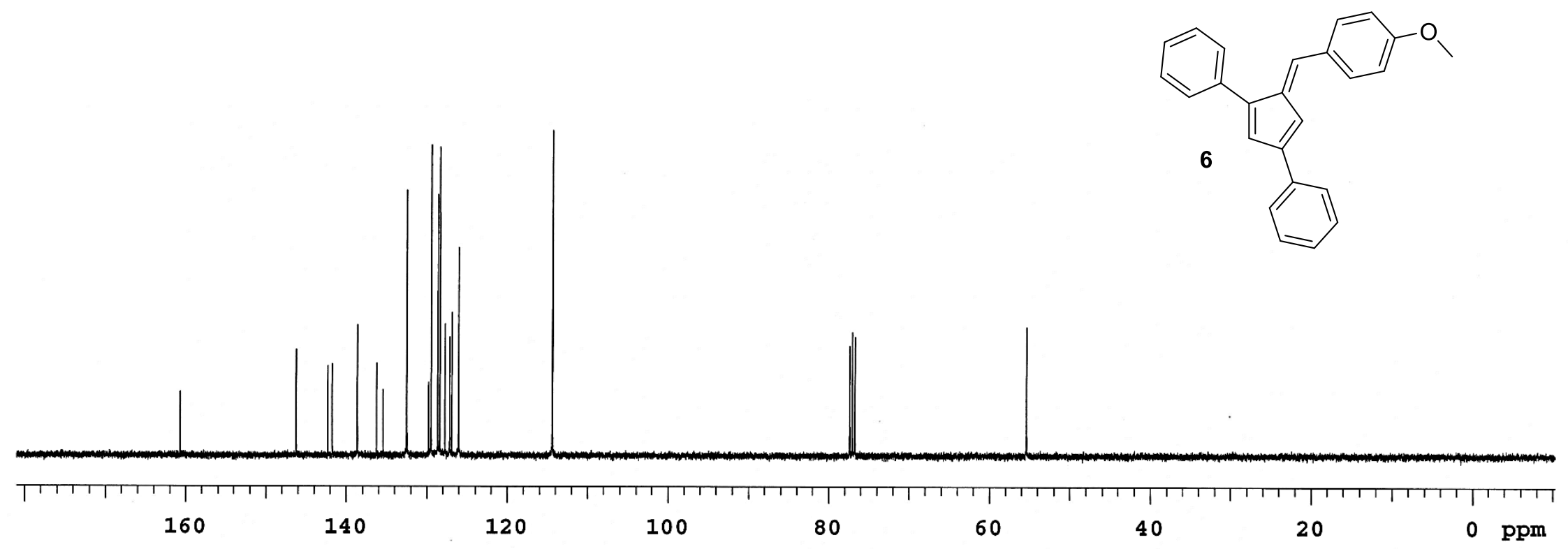


1,3-diphenyl-6-(4-methylphenyl)fulvene (7)

${ }^{1}$ H-NMR (400 MHz, $\mathrm{CDCl}_{3}$ )

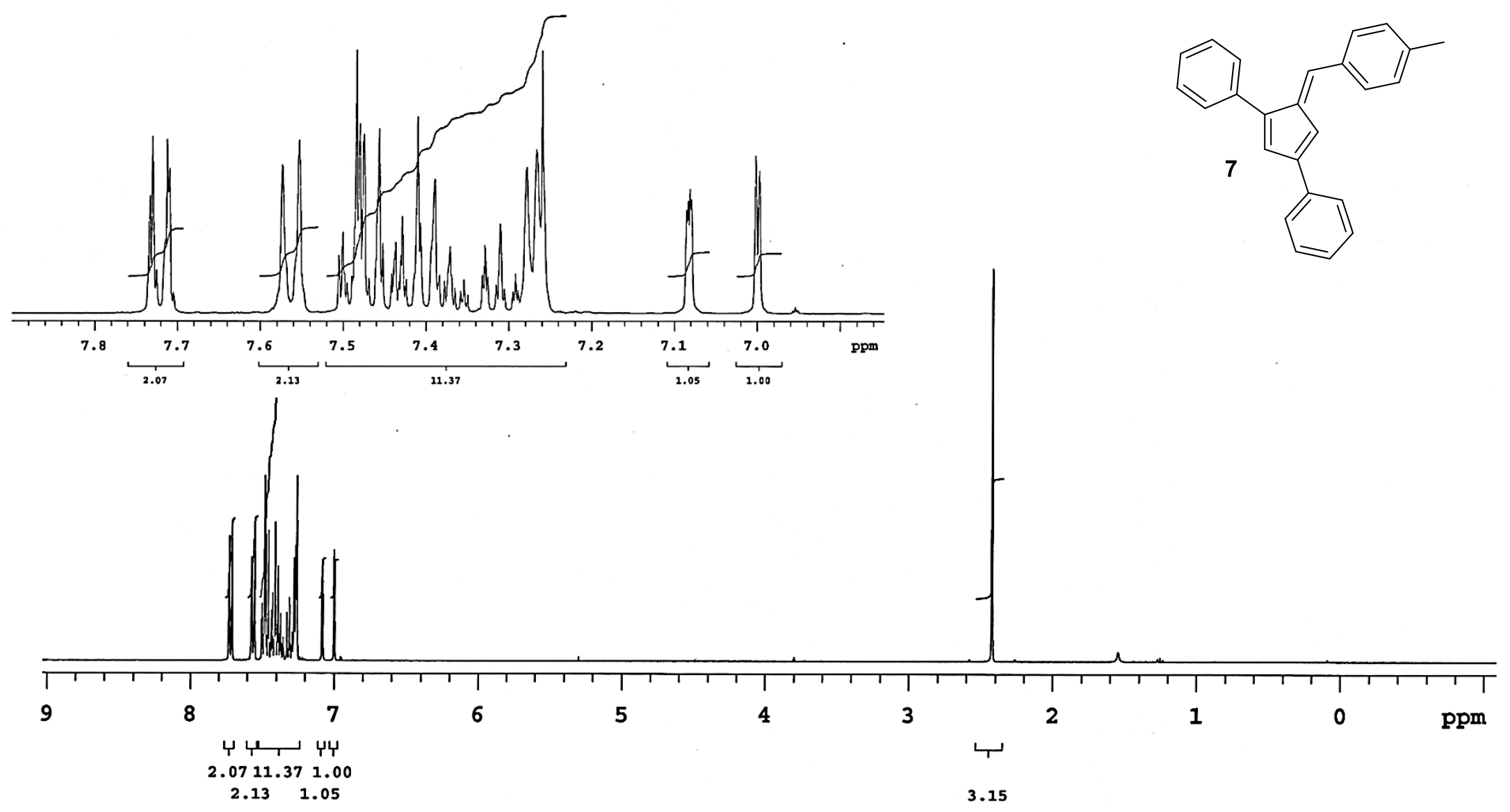


1,3-diphenyl-6-(4-methylphenyl)fulvene (7)

${ }^{13} \mathrm{C}$-NMR (100 $\left.\mathrm{MHz}, \mathrm{CDCl}_{3}\right)$

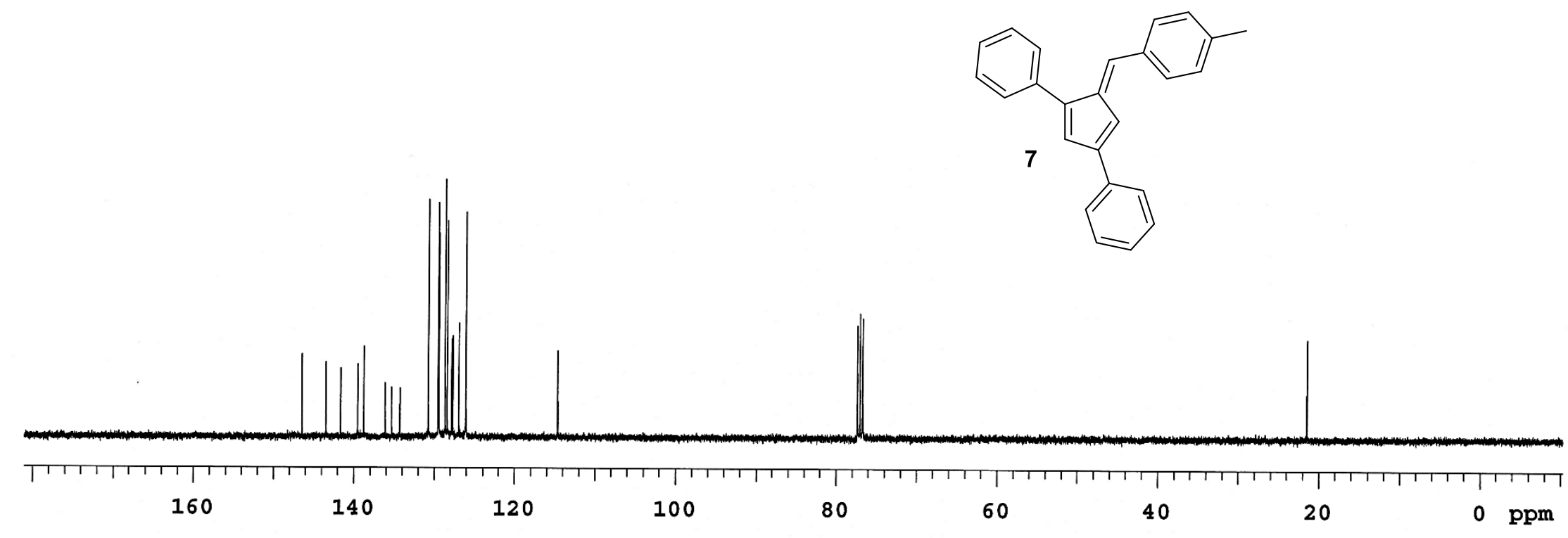


1,3-diphenyl-6-(4-bipheny)lfulvene (9)

${ }^{1}$ H-NMR (400 MHz, $\mathrm{CDCl}_{3}$ )

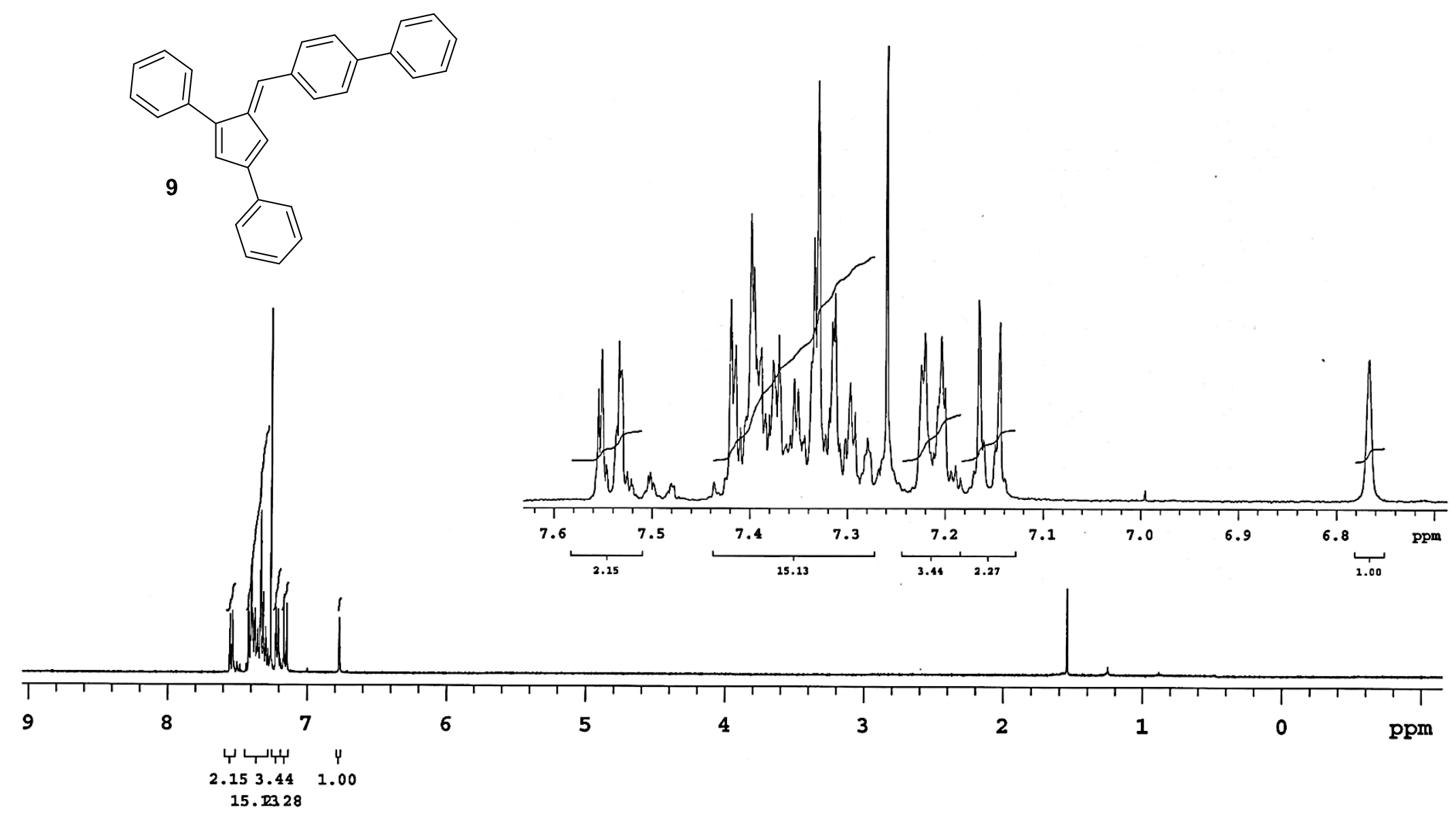


1,3-diphenyl-6-(4-biphenyl)fulvene (9)

${ }^{13} \mathrm{C}$-NMR (100 $\mathrm{MHz}, \mathrm{CDCl}_{3}$ )

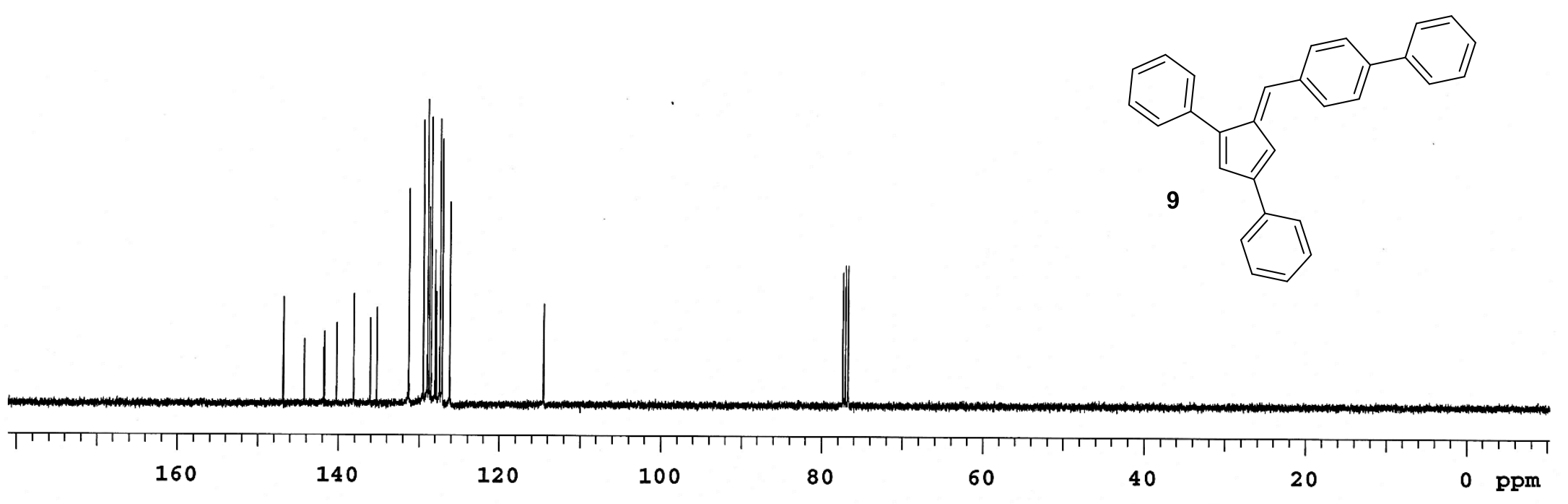


1,3-diphenyl-6-(3-methylphenyl)lfulvene (10)

1H-NMR (400 MHz, CDCI3)

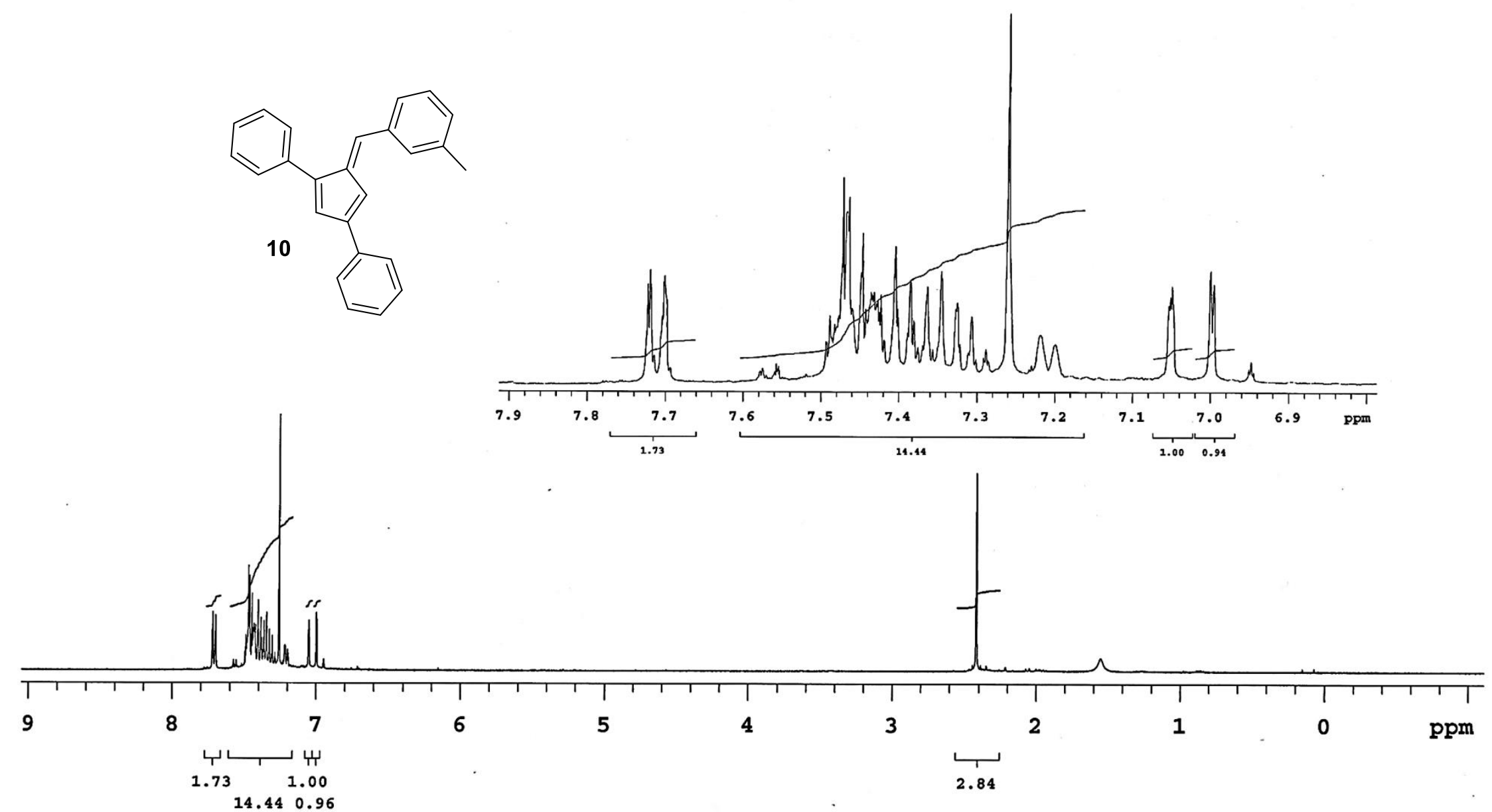


1,3-diphenyl-6-(3-methylphenyl)lfulvene (10)

${ }^{13} \mathrm{C}$-NMR (100 $\left.\mathrm{MHz}, \mathrm{CDCl}_{3}\right)$

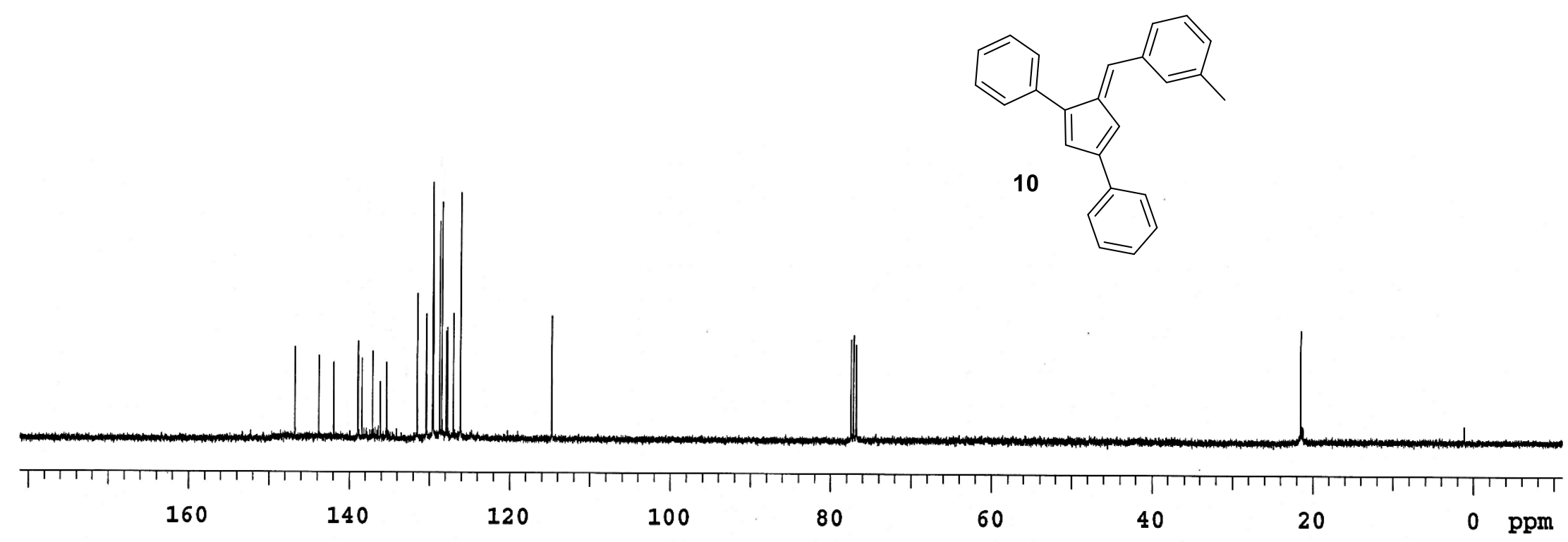


1,3-diphenyl-6-(4-fluorophenyl)fulvene (11)

${ }^{1} \mathrm{H}-\mathrm{NMR}$ (400 MHz, $\mathrm{CDCl}_{3}$ )

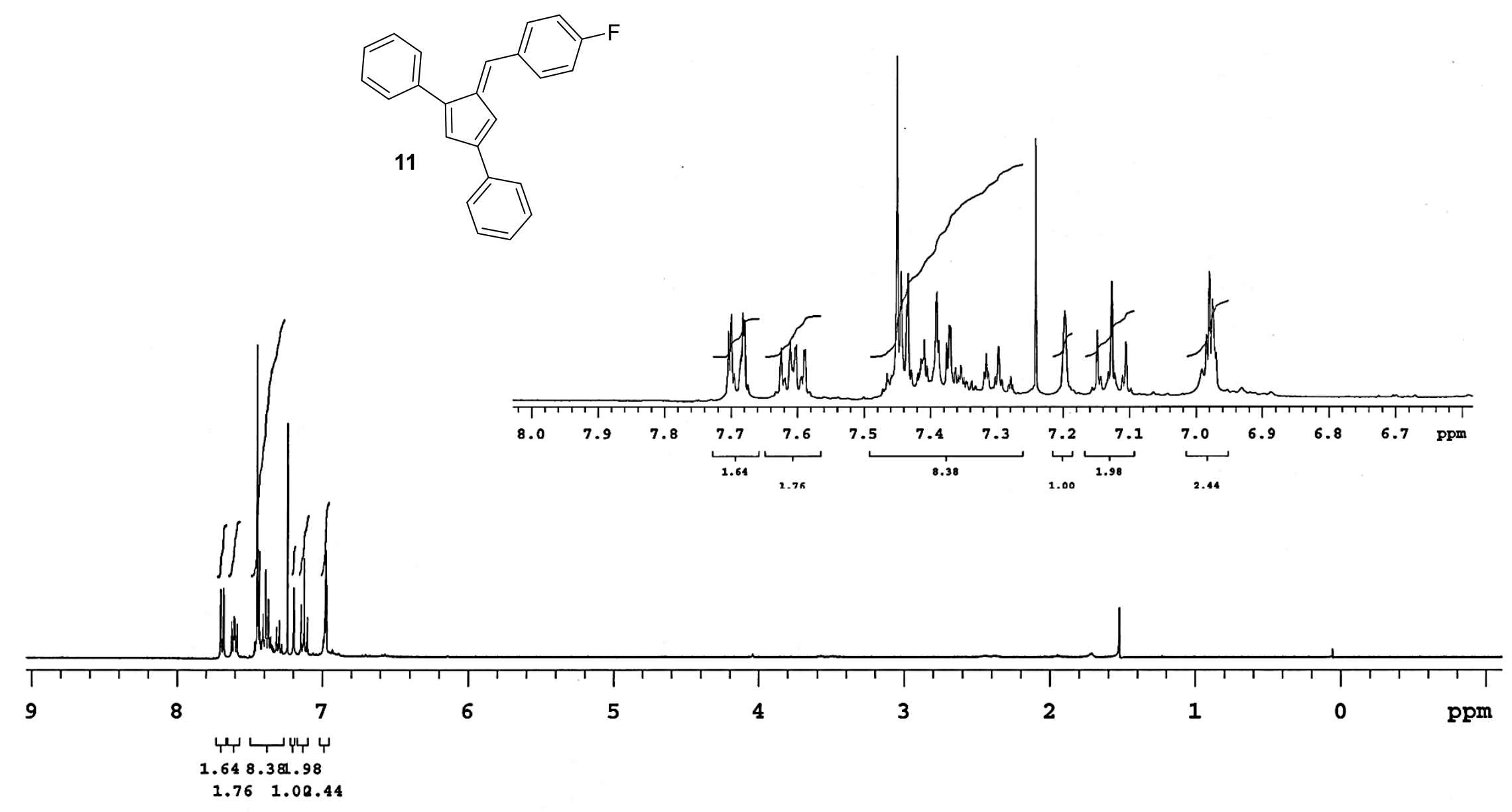


1,3-diphenyl-6-(4-fluorophenyl)fulvene (11)

${ }^{13} \mathrm{C}$-NMR (100 $\left.\mathrm{MHz}, \mathrm{CDCl}_{3}\right)$

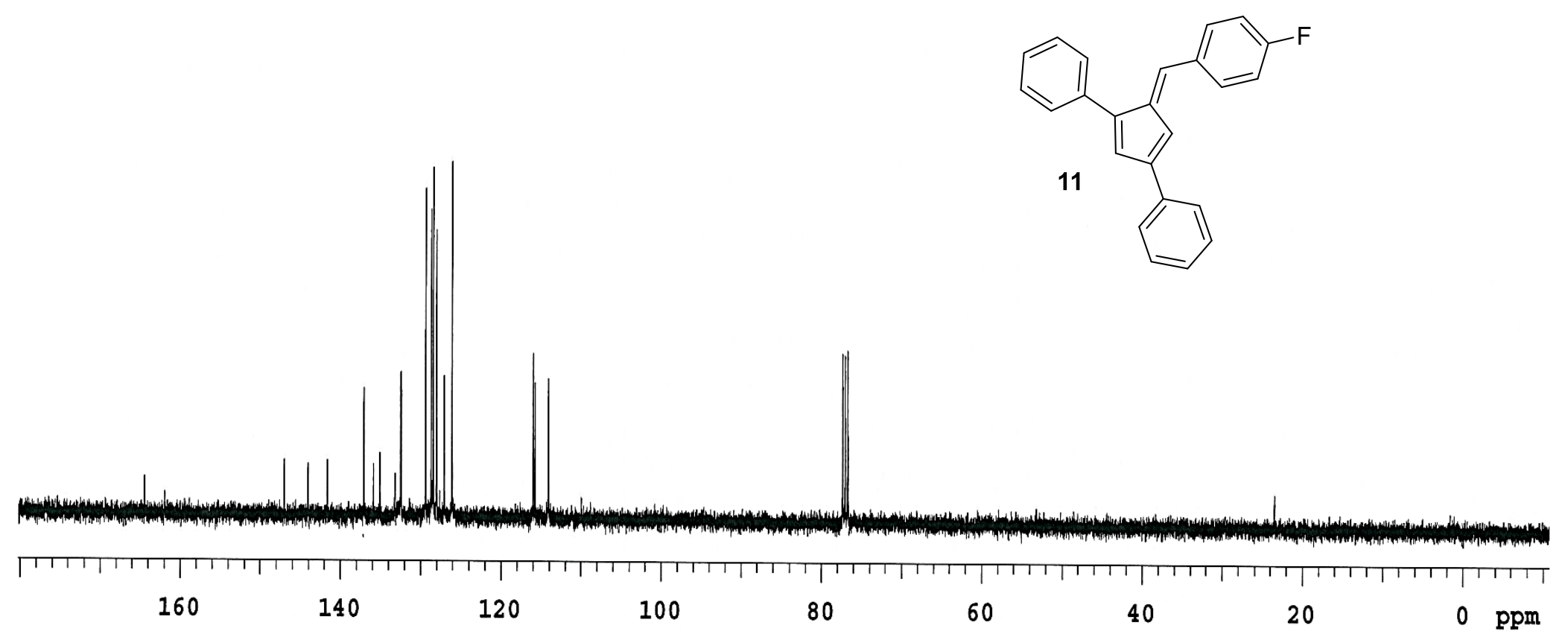


1,3-diphenyl-6-(4-fluorophenyl)fulvene (11)

${ }^{19}$ F-NMR (375 MHz, CDCl $)$

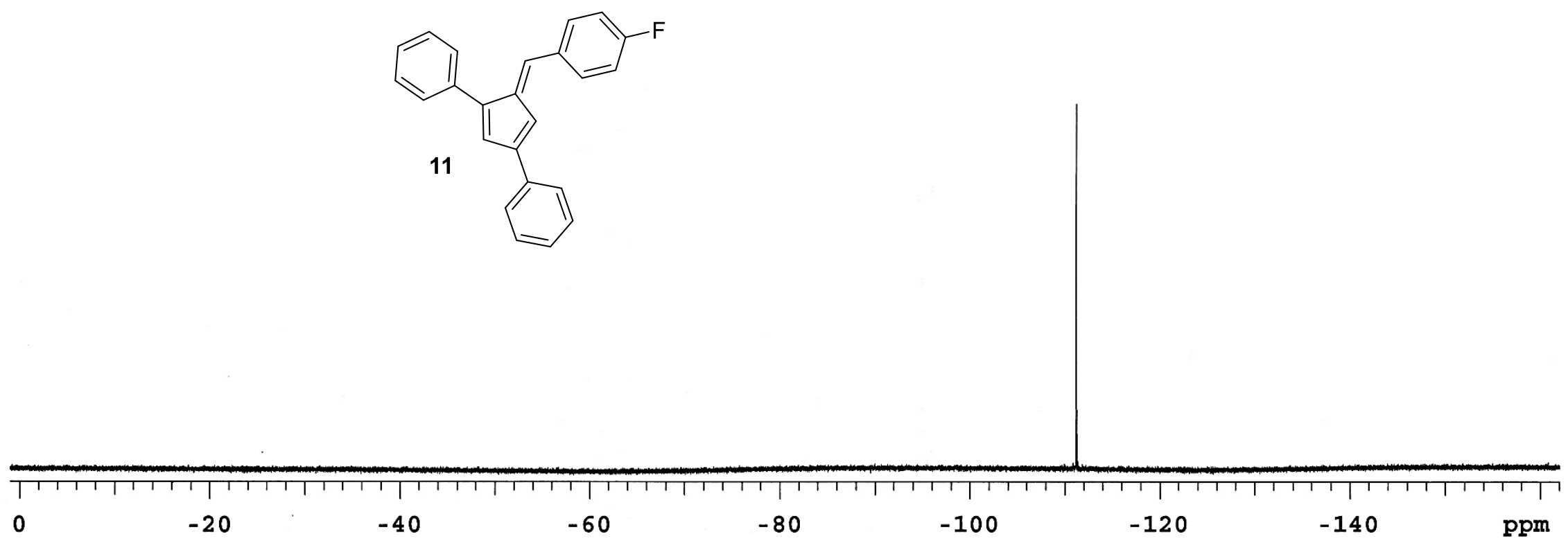


1,3-diphenyl-6-(3-methoxyphenyl)fulvene (12)

1H-NMR (400 MHz, CDCl3)

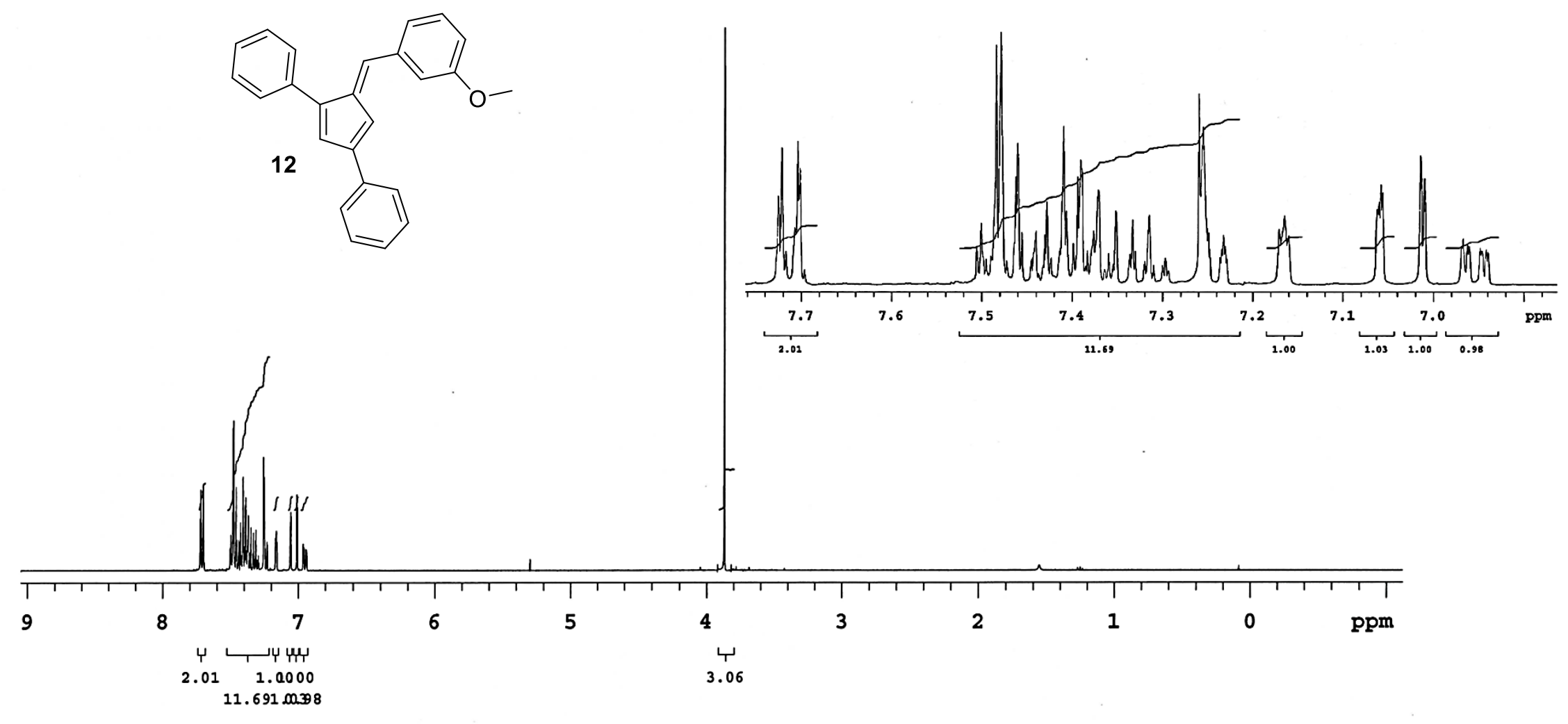


1,3-diphenyl-6-(3-methoxyphenyl)fulvene (12)

${ }^{13} \mathrm{C}$-NMR (100 MHz, $\left.\mathrm{CDCl}_{3}\right)$

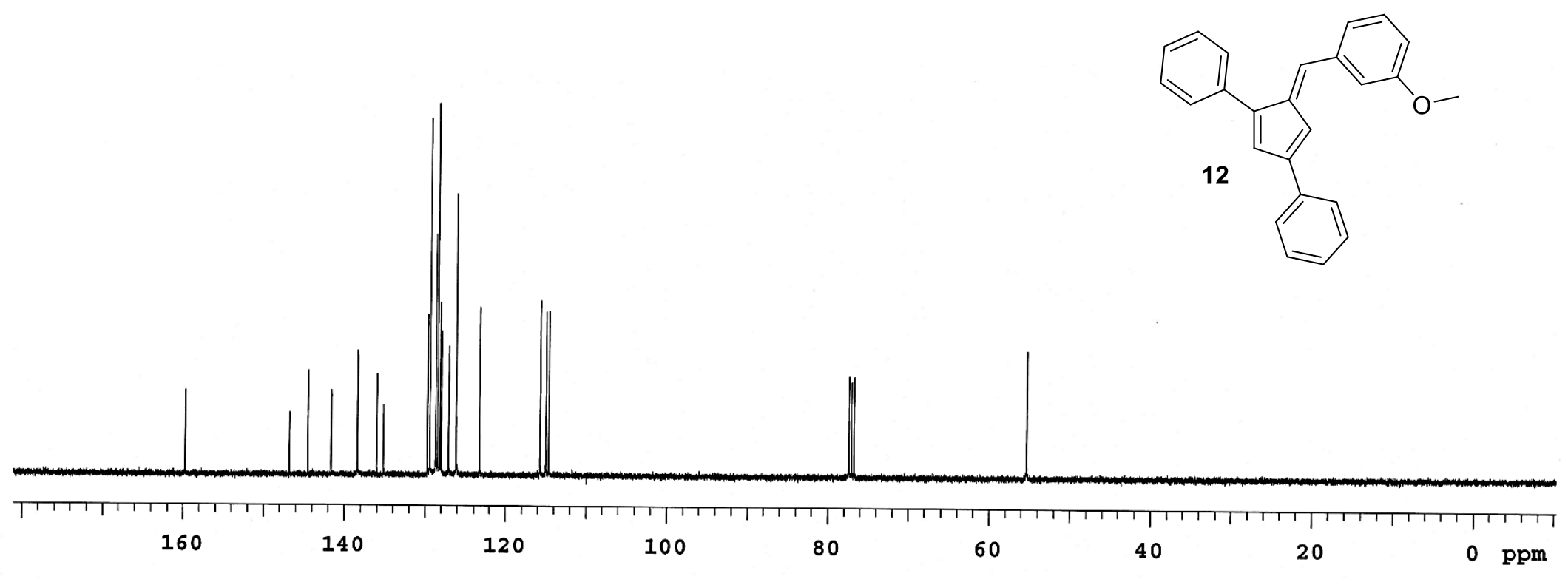


1,3-diphenyl-6-[4-(2-pyridyl)phenyl]fulvene (14)

${ }^{1} \mathrm{H}-\mathrm{NMR}$ (400 MHz, $\mathrm{CDCl}_{3}$ )

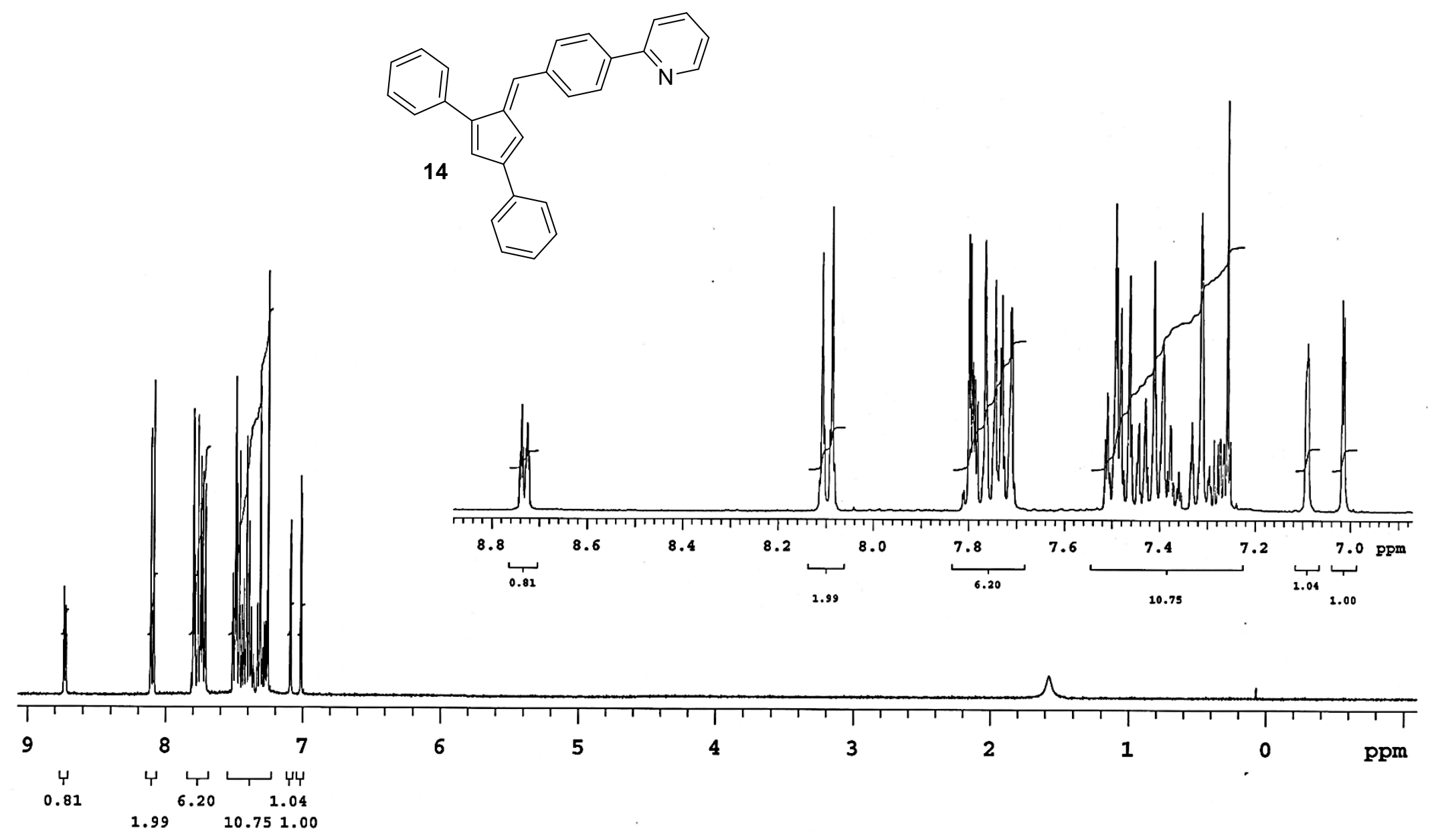


1,3-diphenyl-6-[4-(2-pyridyl)phenyl]fulvene (14)

${ }^{13} \mathrm{C}$-NMR (100 MHz, $\left.\mathrm{CDCl}_{3}\right)$

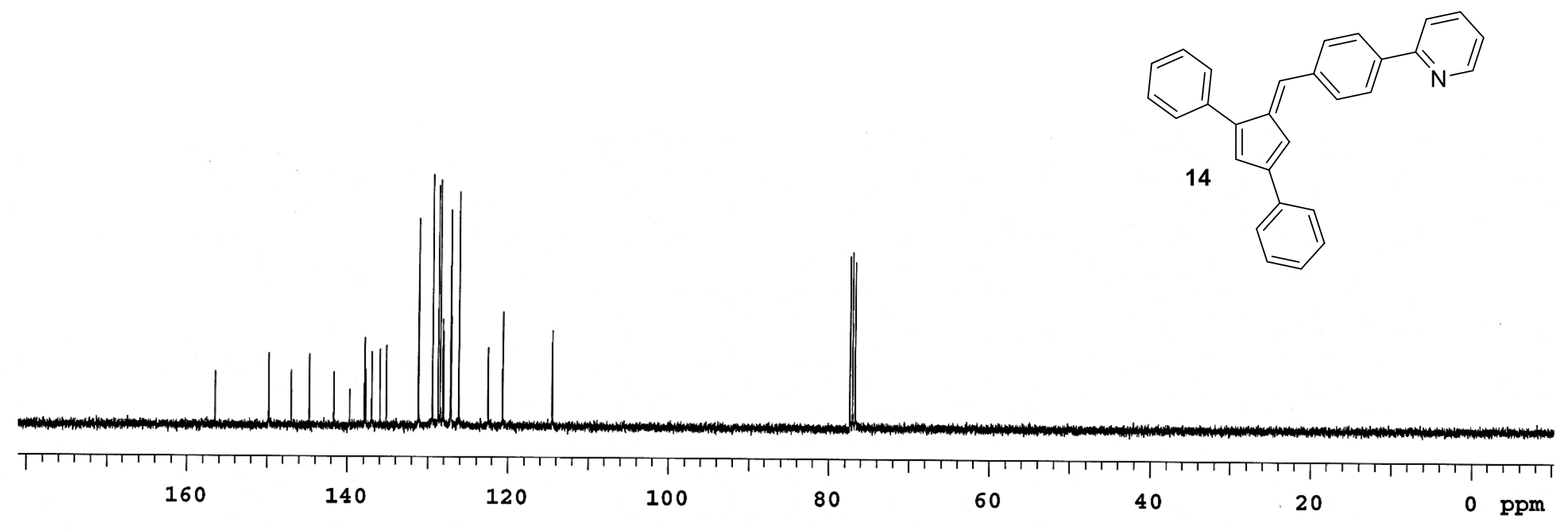


1,3-diphenyl-6-(4-bromophenyl)fulvene (15)

${ }^{1}$ H-NMR (400 MHz, CDCl 3 )

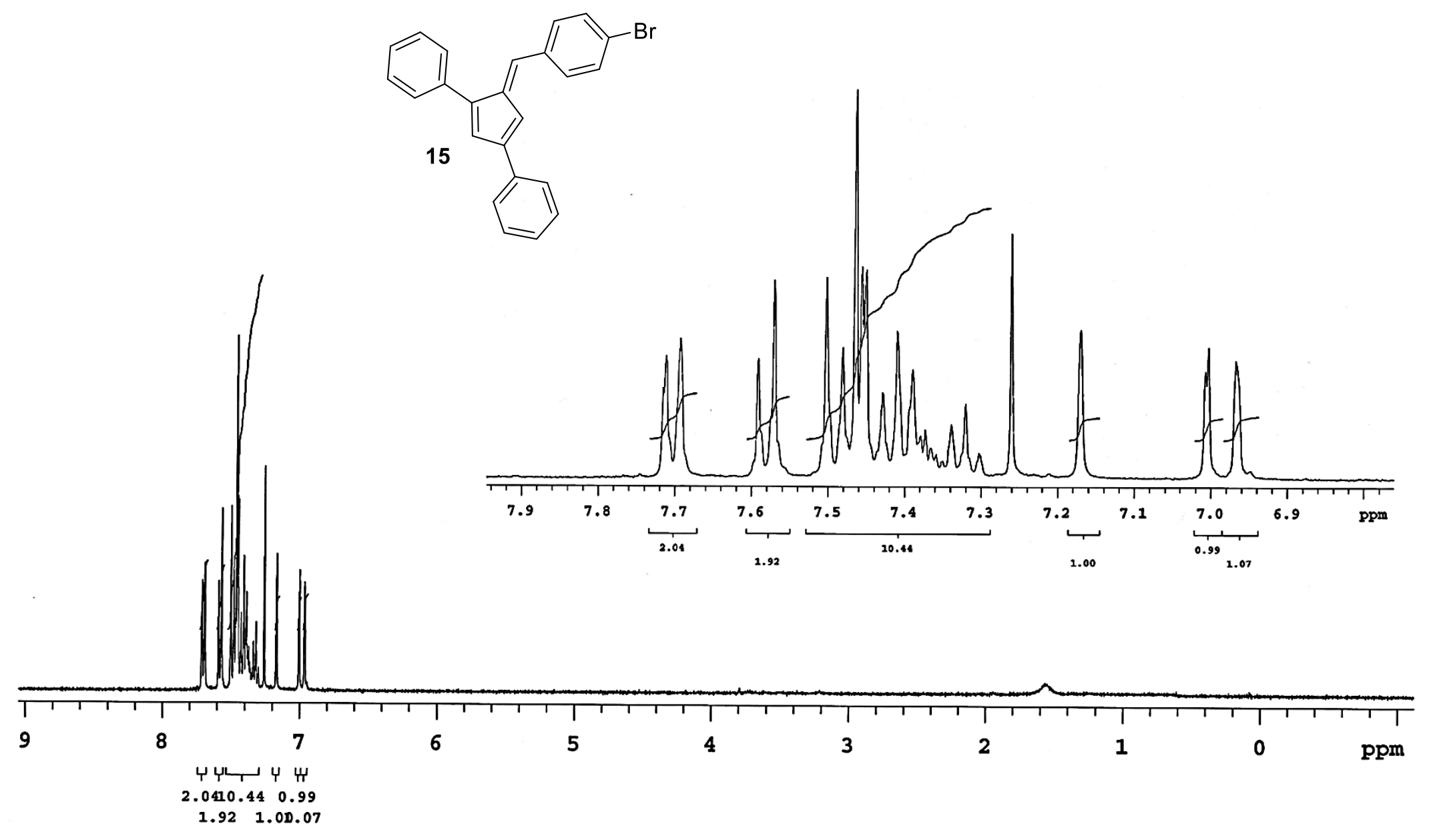


1,3-diphenyl-6-(4-bromophenyl)fulvene (15)

${ }^{13} \mathrm{C}$-NMR (100 $\left.\mathrm{MHz}, \mathrm{CDCl}_{3}\right)$

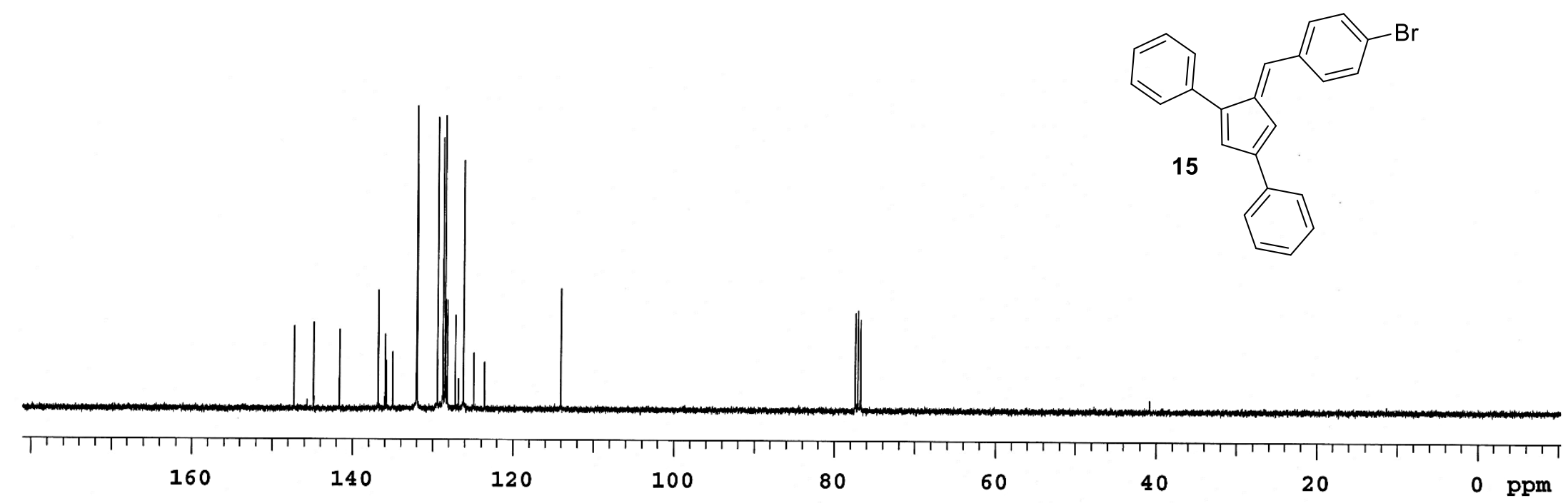


1,3-diphenyl-6-(4-trifluoromethoxyphenyl)fulvene (16)

${ }^{1} \mathrm{H}-\mathrm{NMR}$ (400 MHz, $\mathrm{CDCl}_{3}$ )

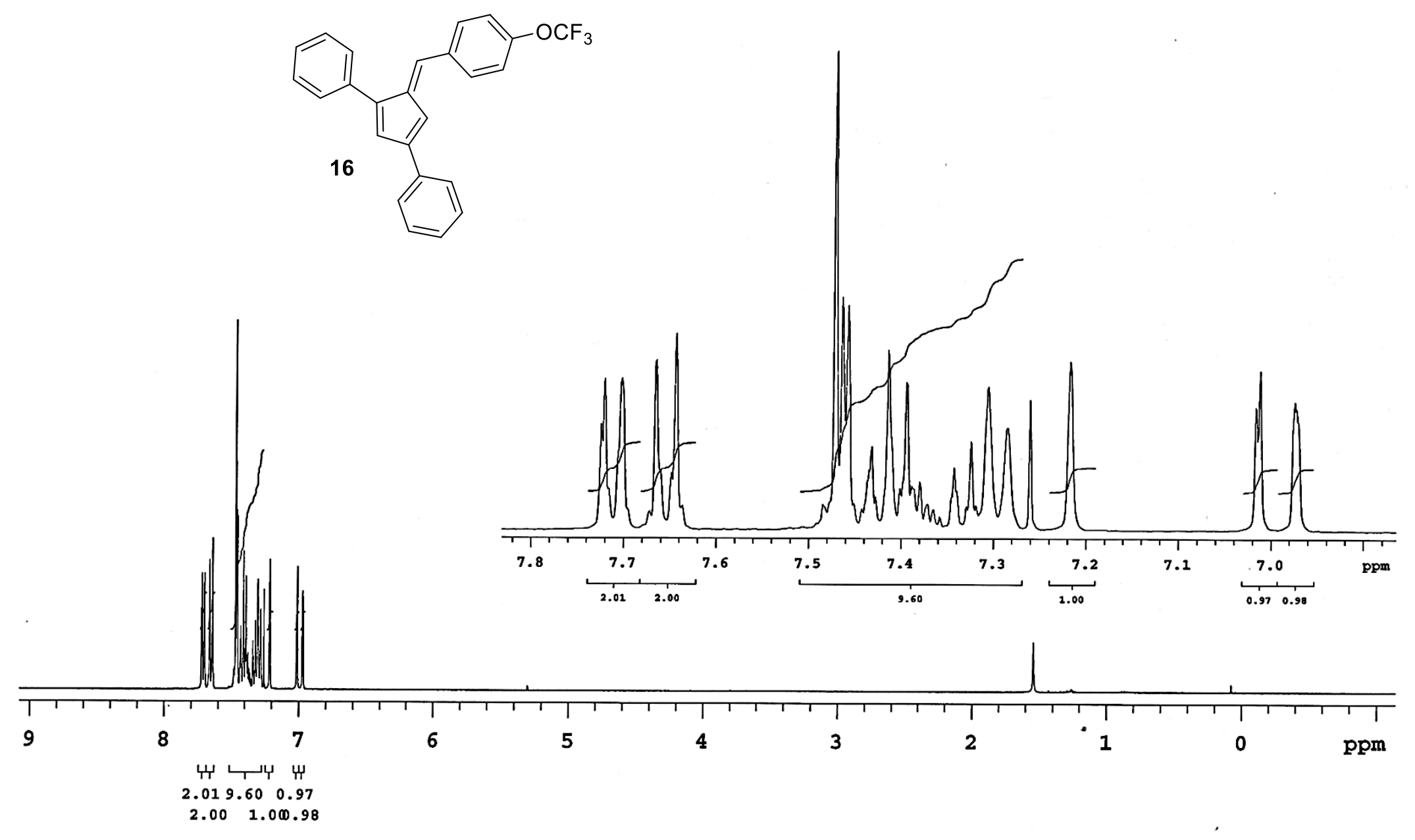


1,3-diphenyl-6-(4-trifluoromethoxyphenyl)fulvene (16)

${ }^{13} \mathrm{C}-\mathrm{NMR}\left(100 \mathrm{MHz}, \mathrm{CDCl}_{3}\right)$

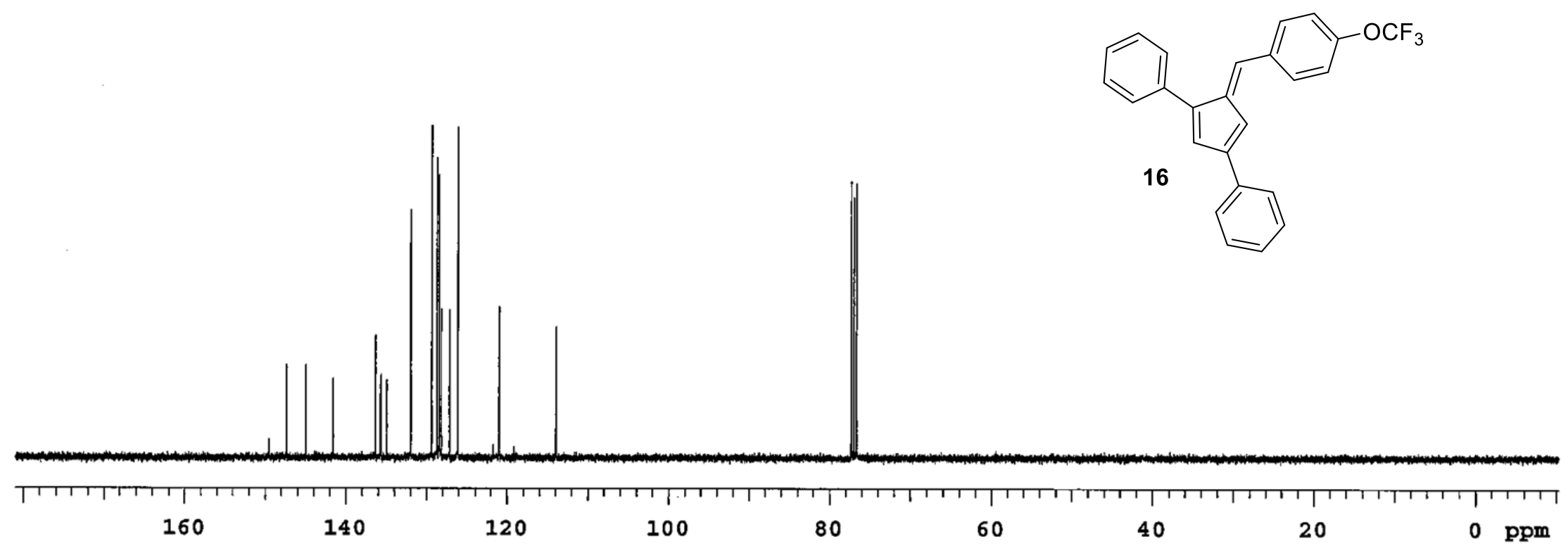


1,3-diphenyl-6-(4-trifluoromethoxyphenyl)fulvene (16)

${ }^{19}$ F-NMR (376 MHz, $\left.\mathrm{CDCl}_{3}\right)$

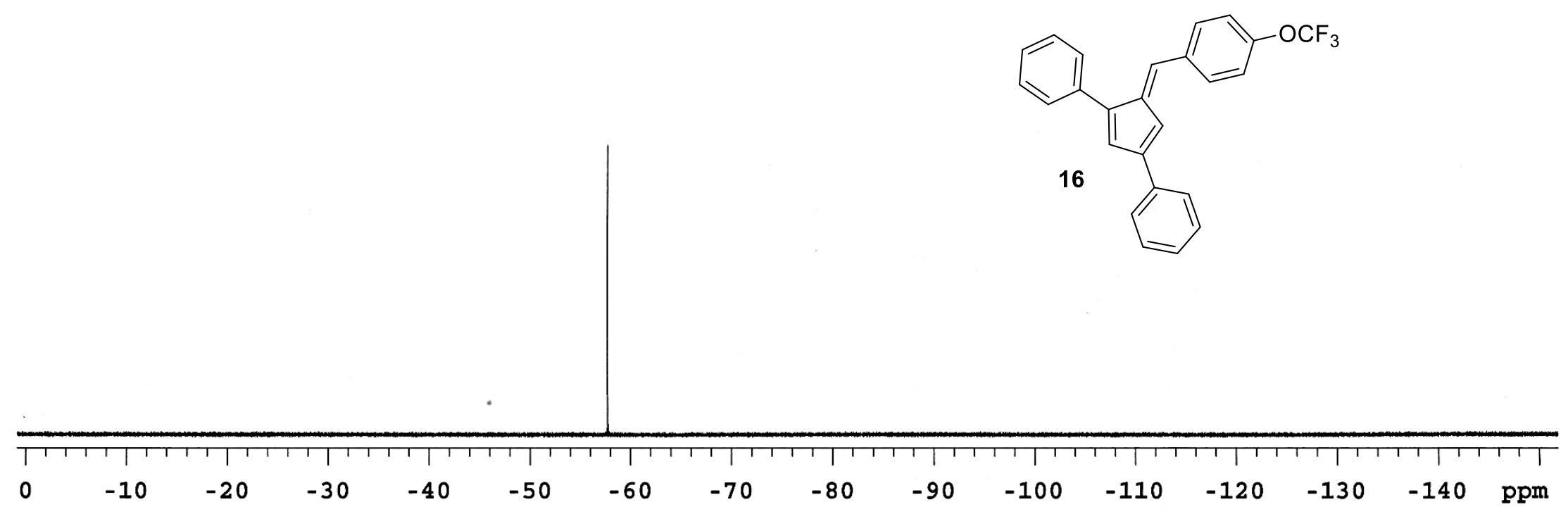


1,3-diphenyl-6-(4-ethynylphenyl)fulvene (17)

${ }^{1} \mathrm{H}-\mathrm{NMR}$ (400 MHz, $\mathrm{CDCl}_{3}$ )

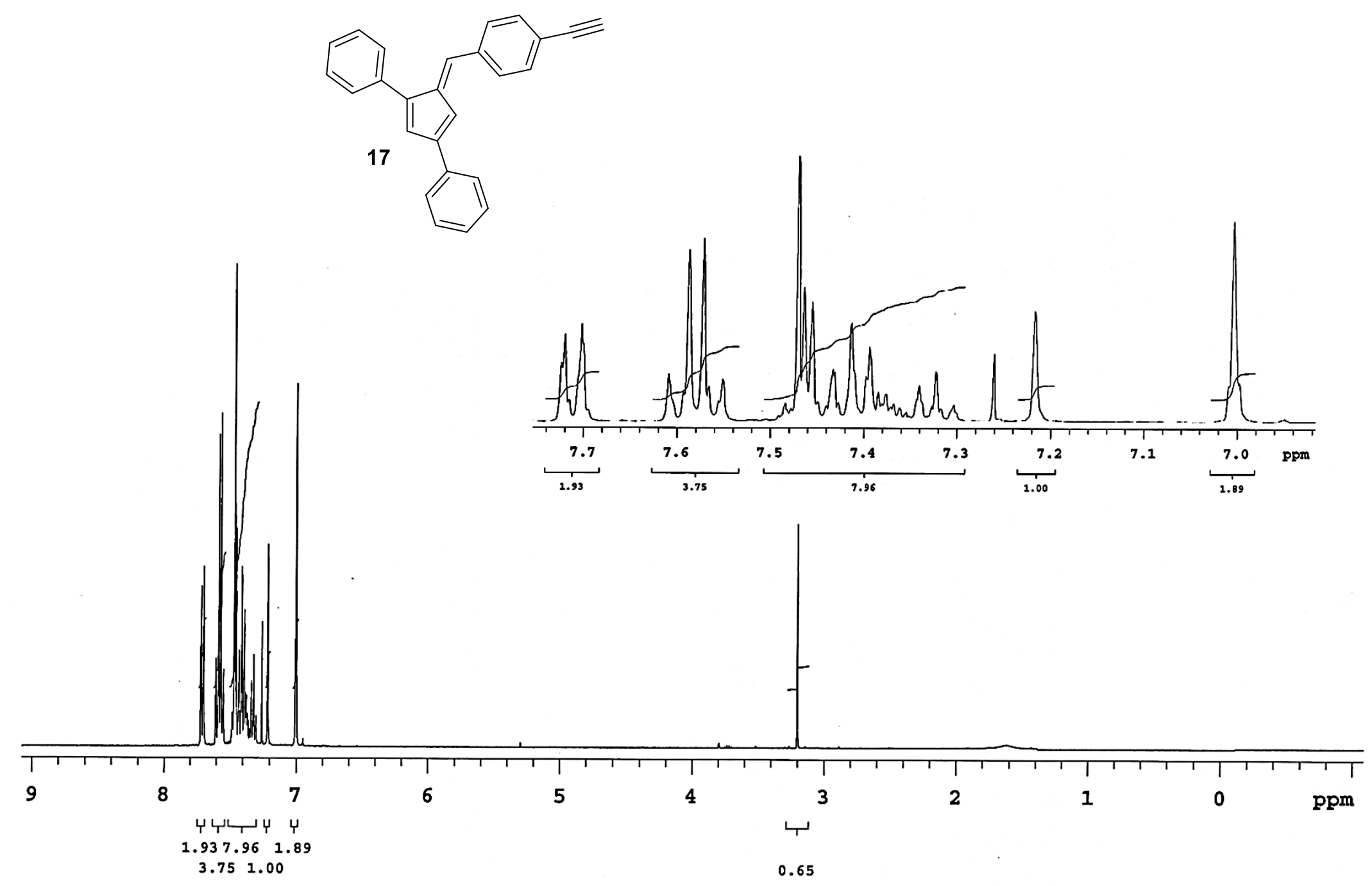


1,3-diphenyl-6-(4-ethynylphenyl)fulvene (17)

${ }^{13} \mathrm{C}$-NMR (100 $\left.\mathrm{MHz}, \mathrm{CDCl}_{3}\right)$

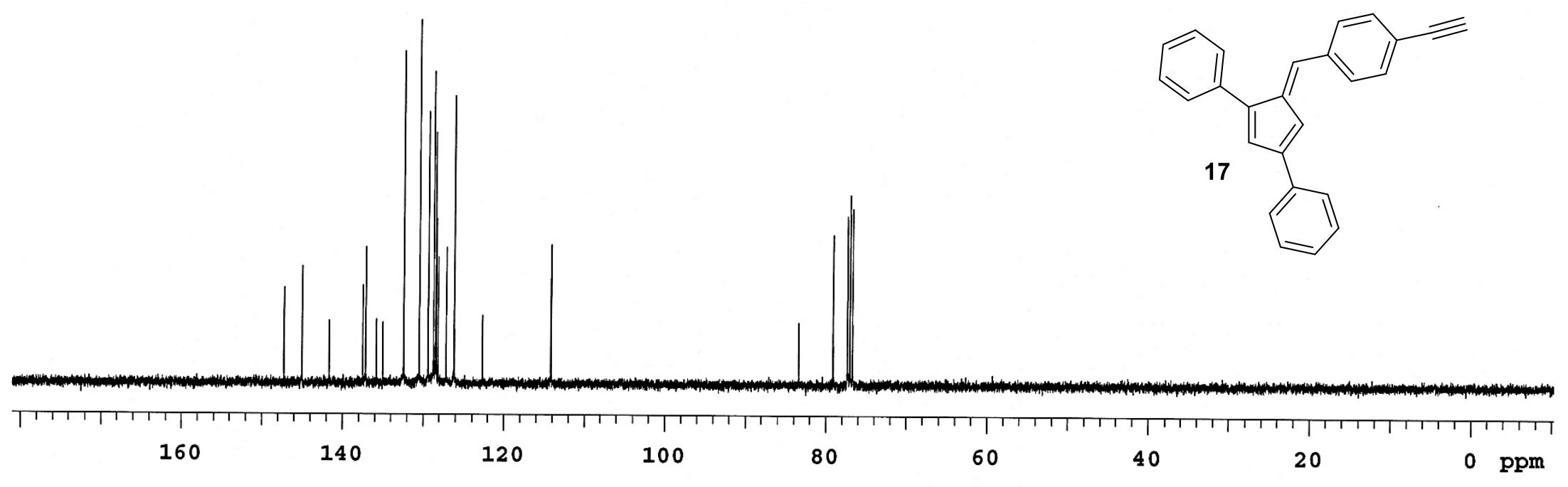


1,3-diphenyl-6-[4-(4-pyridyl)phenyl]fulvene (18)

${ }^{1}$ H-NMR (400 MHz, CDCl $)$

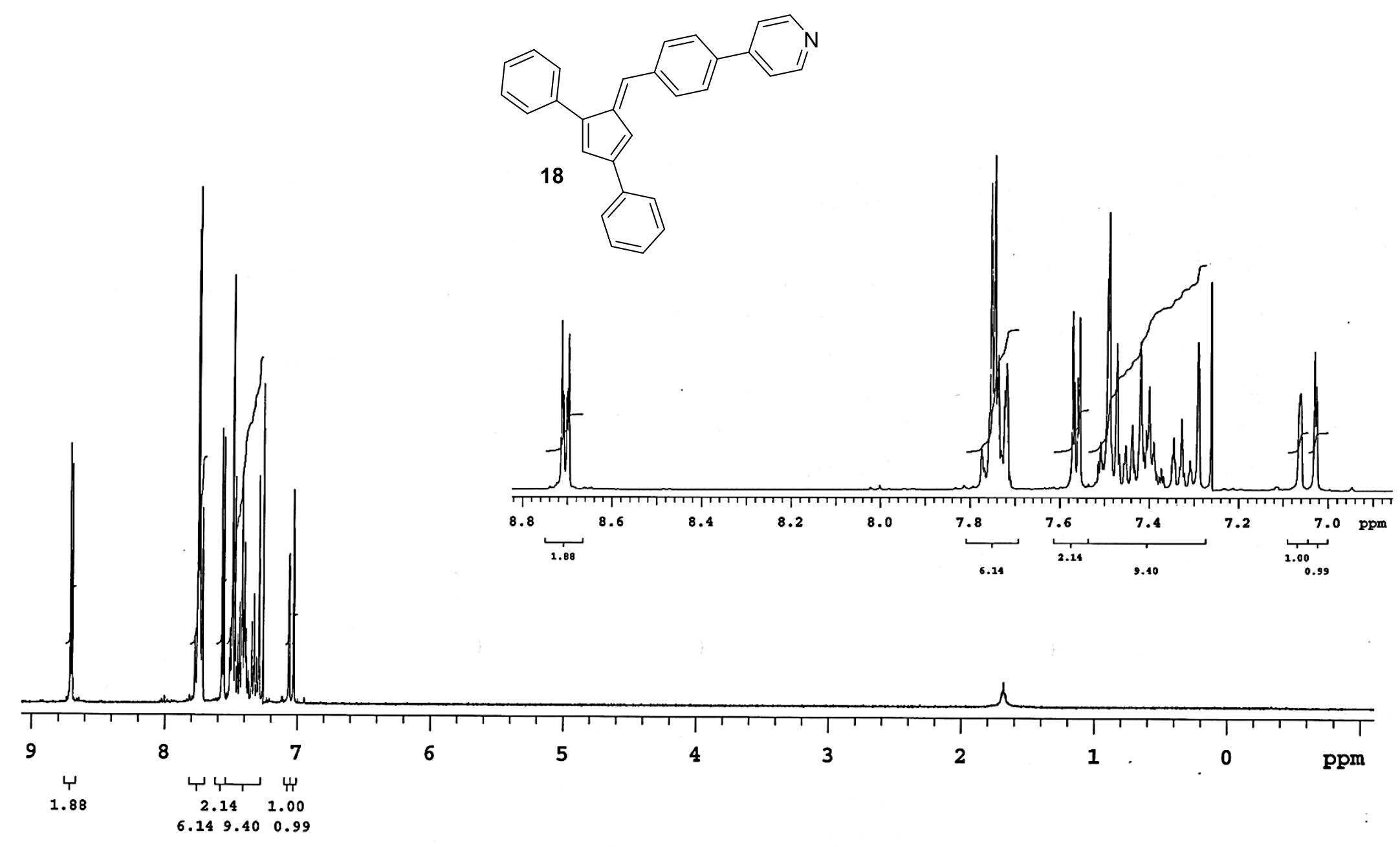


1,3-diphenyl-6-[4-(4-pyridyl)phenyl]fulvene (18)

${ }^{13} \mathrm{C}$-NMR (100 MHz, $\left.\mathrm{CDCl}_{3}\right)$

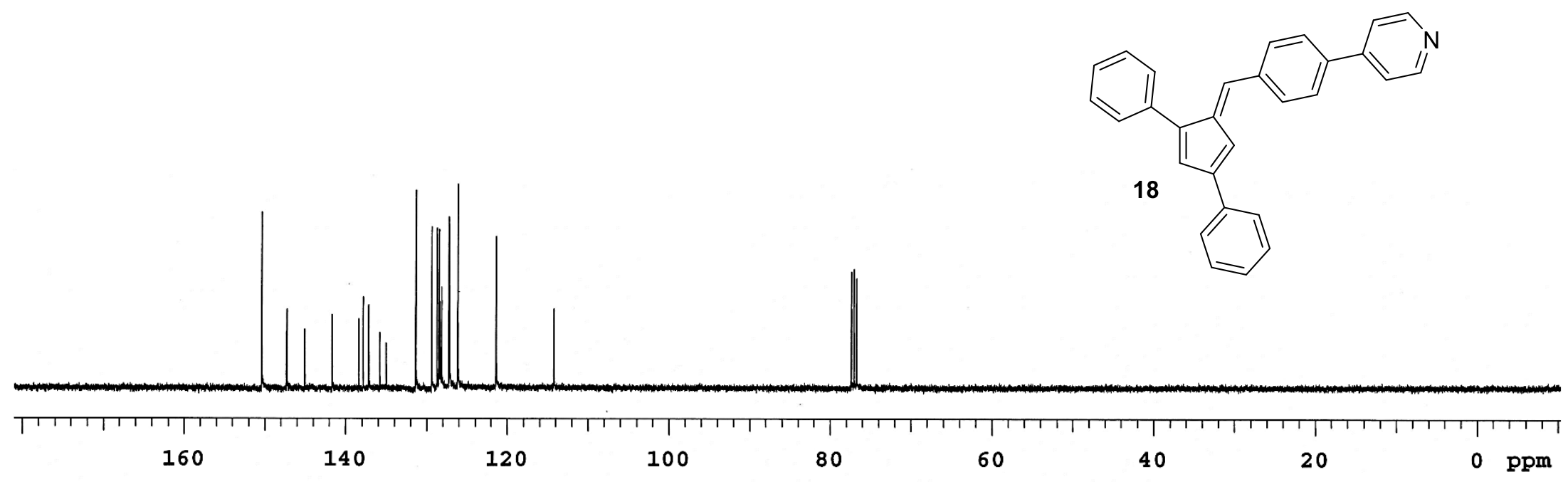


1,3-diphenyl-6-(4-methyl benzoate)fulvene (19)

${ }^{1}$ H-NMR (400 MHz, $\mathrm{CDCl}_{3}$ )

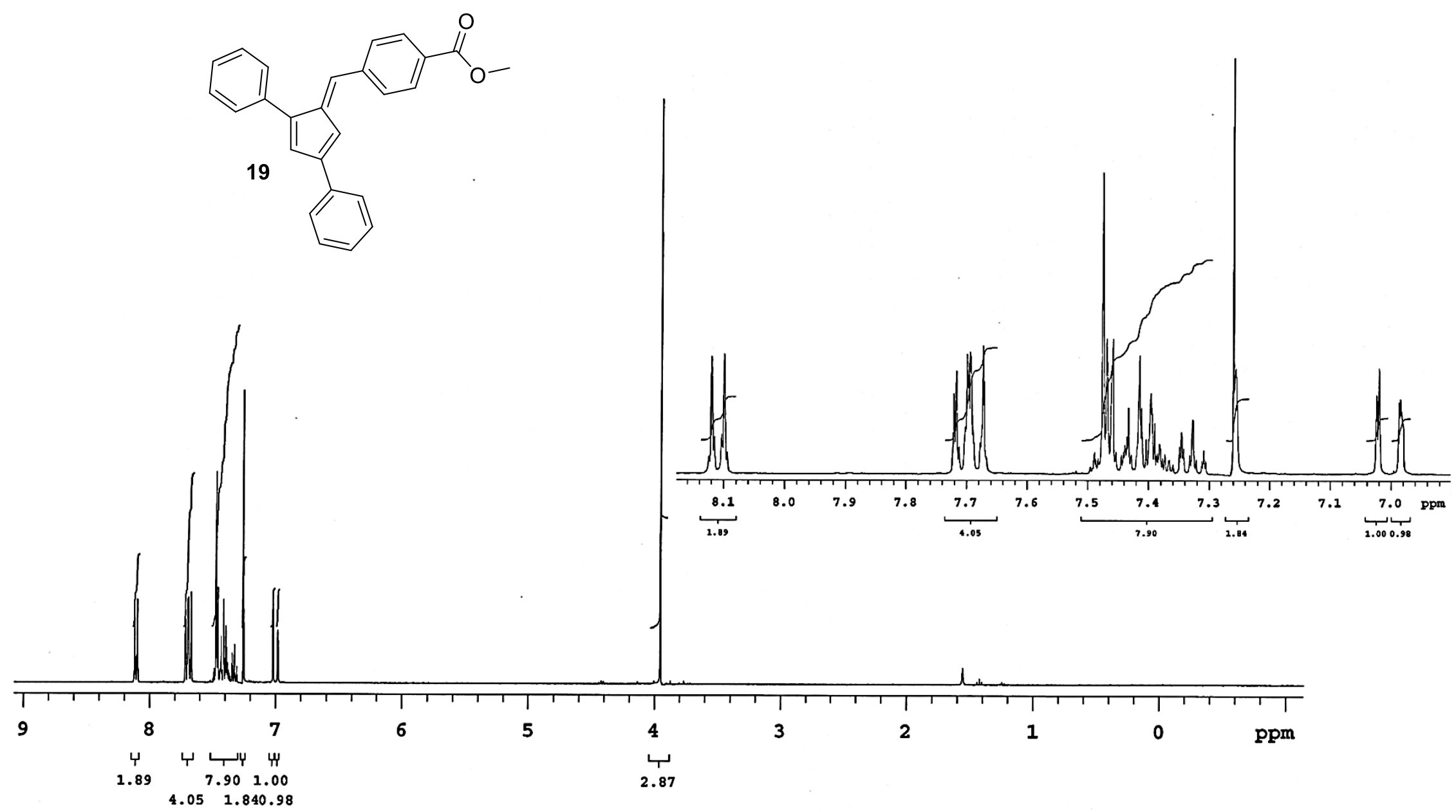


1,3-diphenyl-6-(4-methyl benzoate)fulvene (19)

${ }^{13} \mathrm{C}-\mathrm{NMR}\left(100 \mathrm{MHz}, \mathrm{CDCl}_{3}\right)$

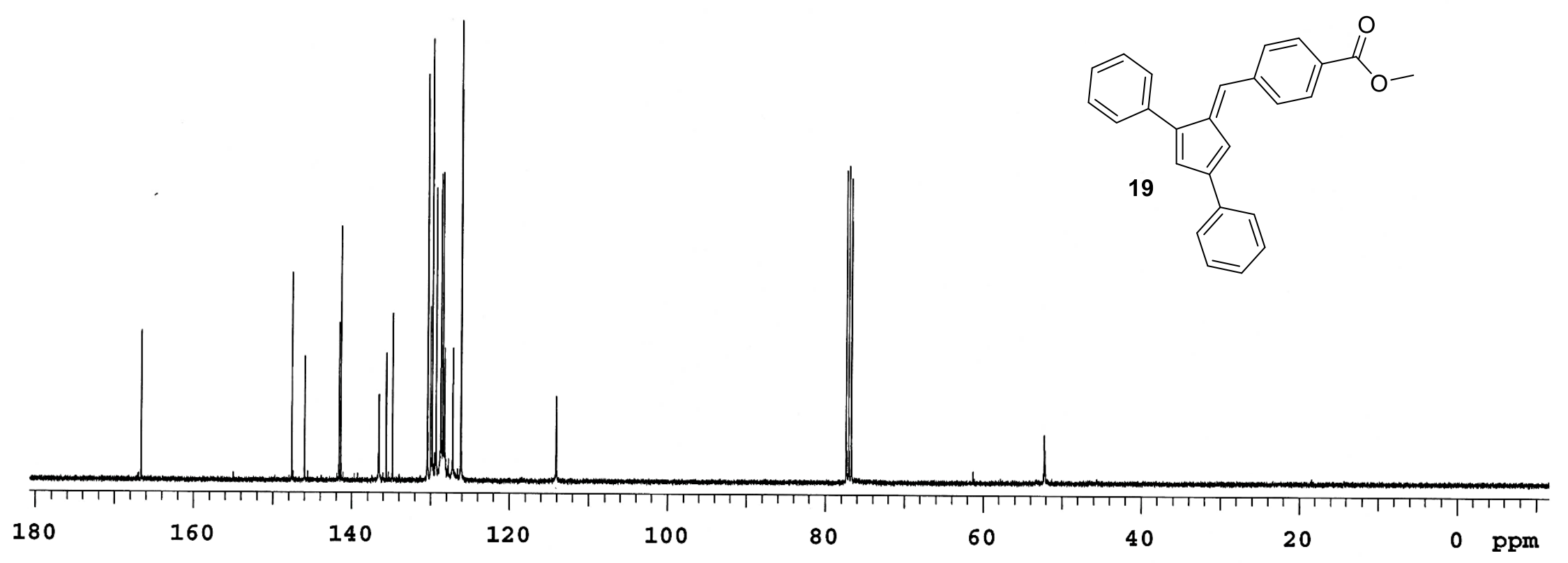


1,3-diphenyl-6-[4-(trimethylammonium iodide)phenyl]fulvene (21)

${ }^{1} \mathrm{H}-\mathrm{NMR}$ (400 MHz, $\mathrm{CDCl}_{3}$ )

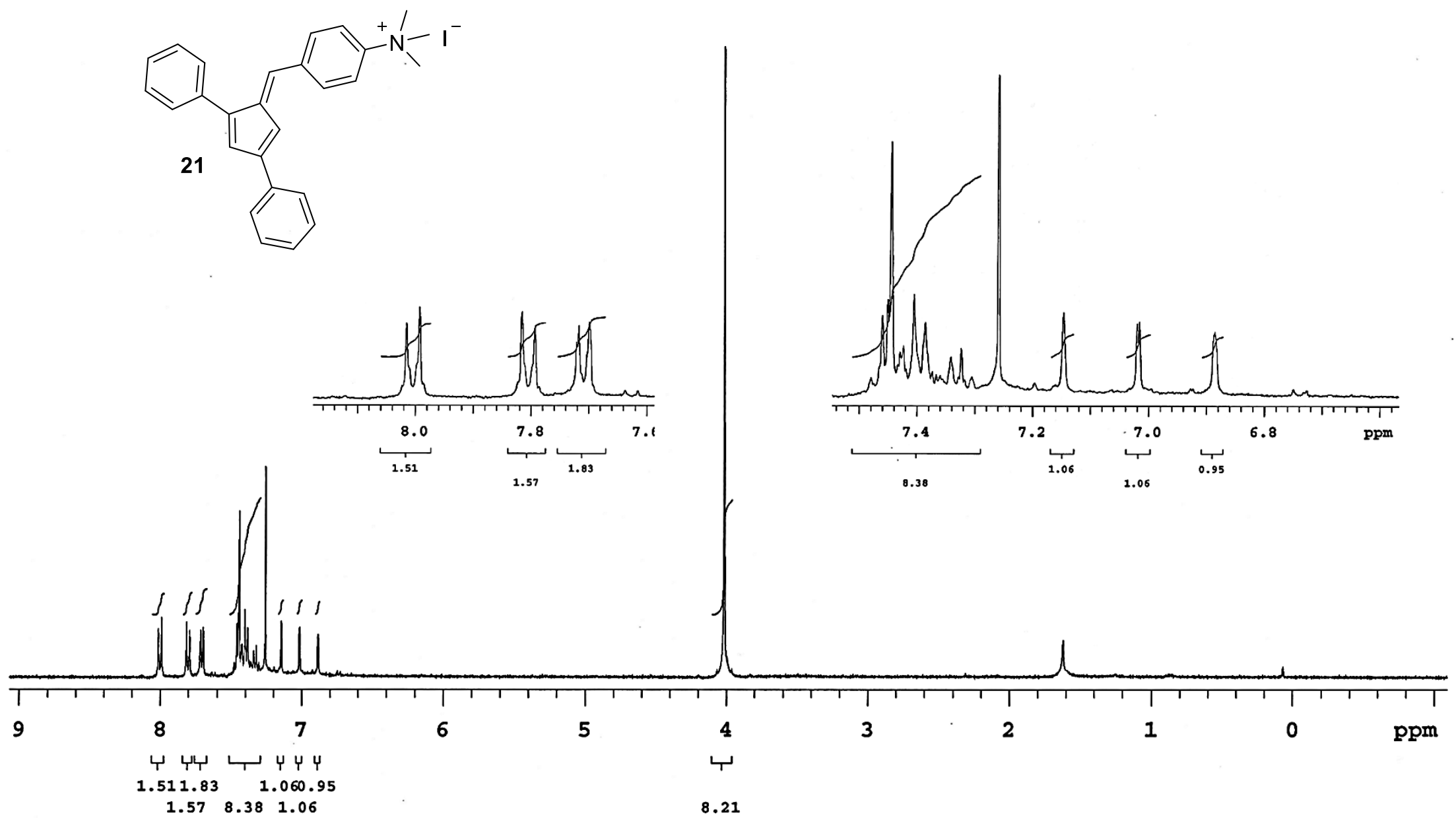


1,3-diphenyl-6-[4-(trimethylammonium iodide)phenyl]fulvene (21)

${ }^{13} \mathrm{C}-\mathrm{NMR}\left(100 \mathrm{MHz}, \mathrm{CDCl}_{3}\right)$

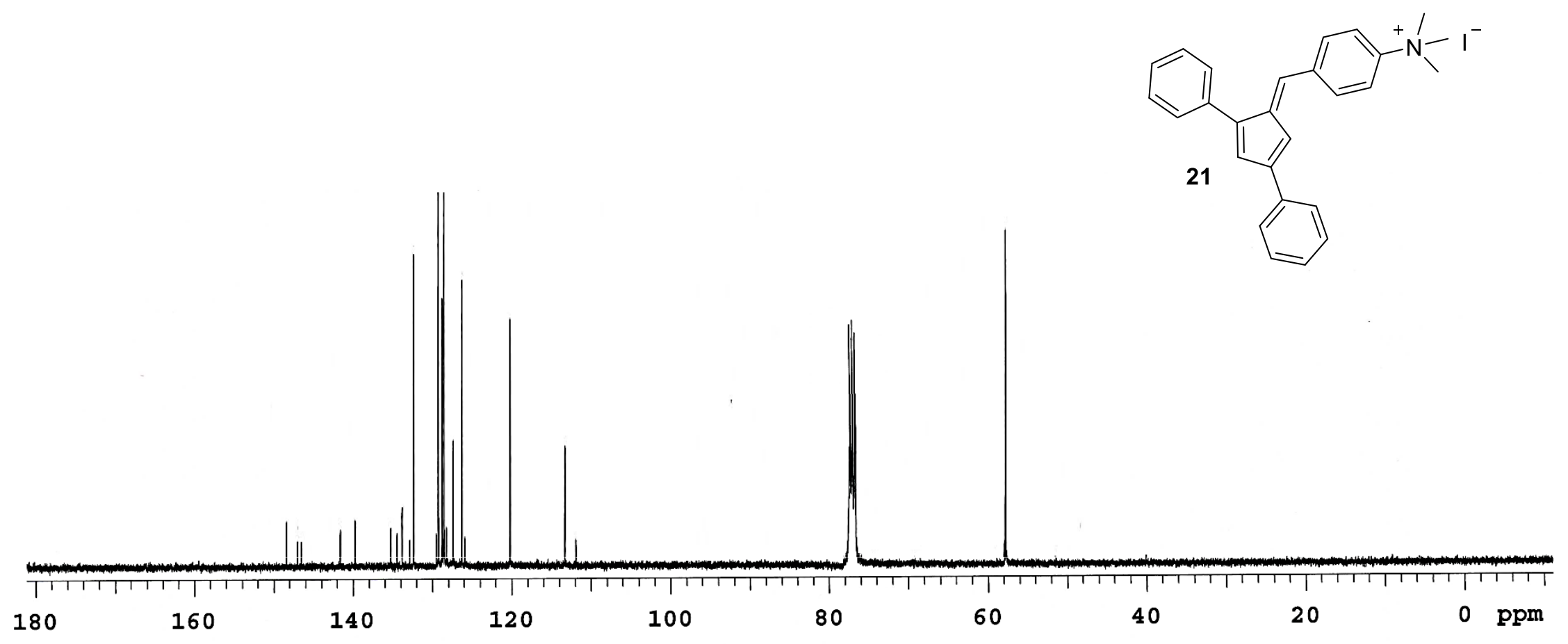


1,3-diphenyl-6-(2-naphthyl)fulvene (22)

1H-NMR (400 MHz, CDCI3)

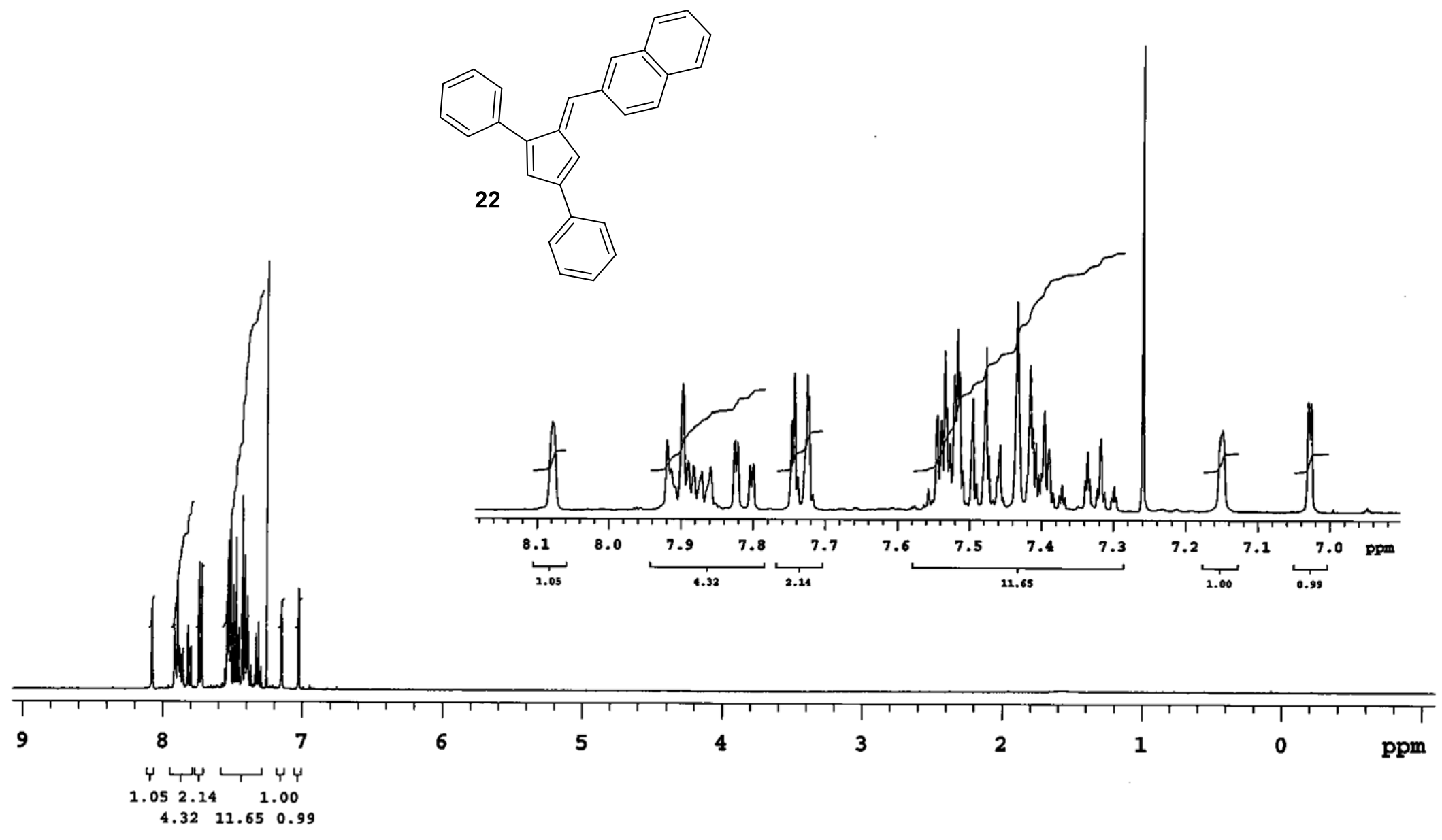


1,3-diphenyl-6-(2-naphthyl)fulvene (22)

${ }^{13} \mathrm{C}$-NMR (100 MHz, $\left.\mathrm{CDCl}_{3}\right)$

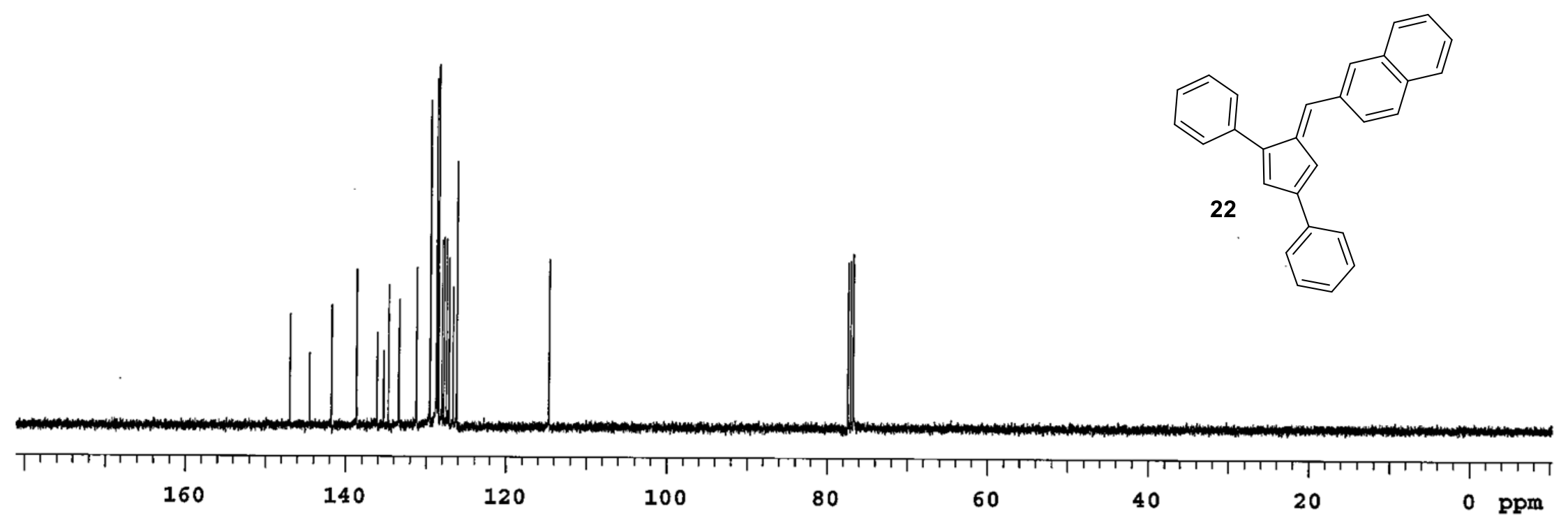


1,3-diphenyl-6-(9-anthracenyl)fulvene (23)

${ }^{1}$ H-NMR (400 MHz, $\mathrm{CDCl}_{3}$ )

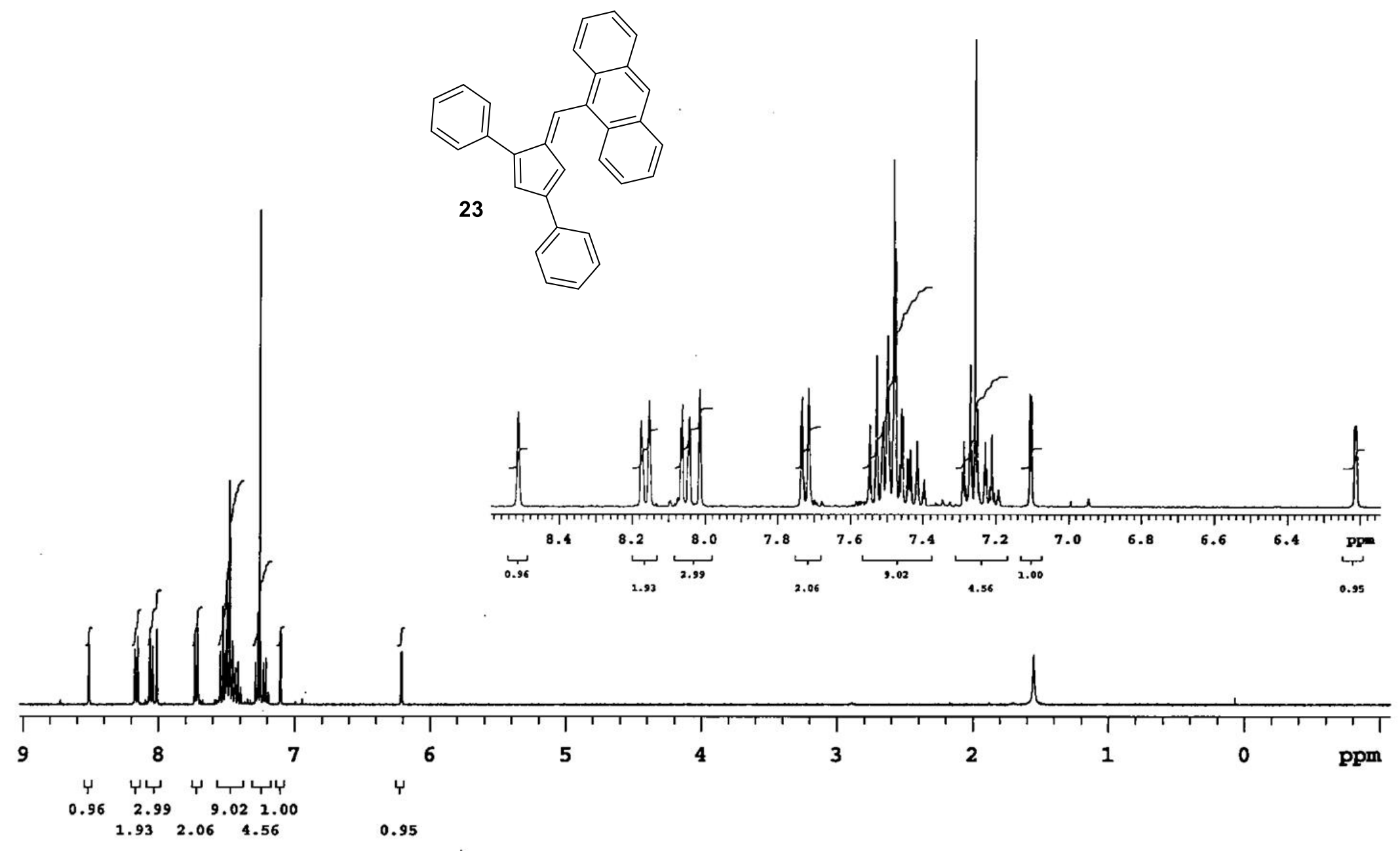


1,3-diphenyl-6-(9-anthracenyl)fulvene (23)

${ }^{13} \mathrm{C}$-NMR (100 MHz, $\left.\mathrm{CDCl}_{3}\right)$

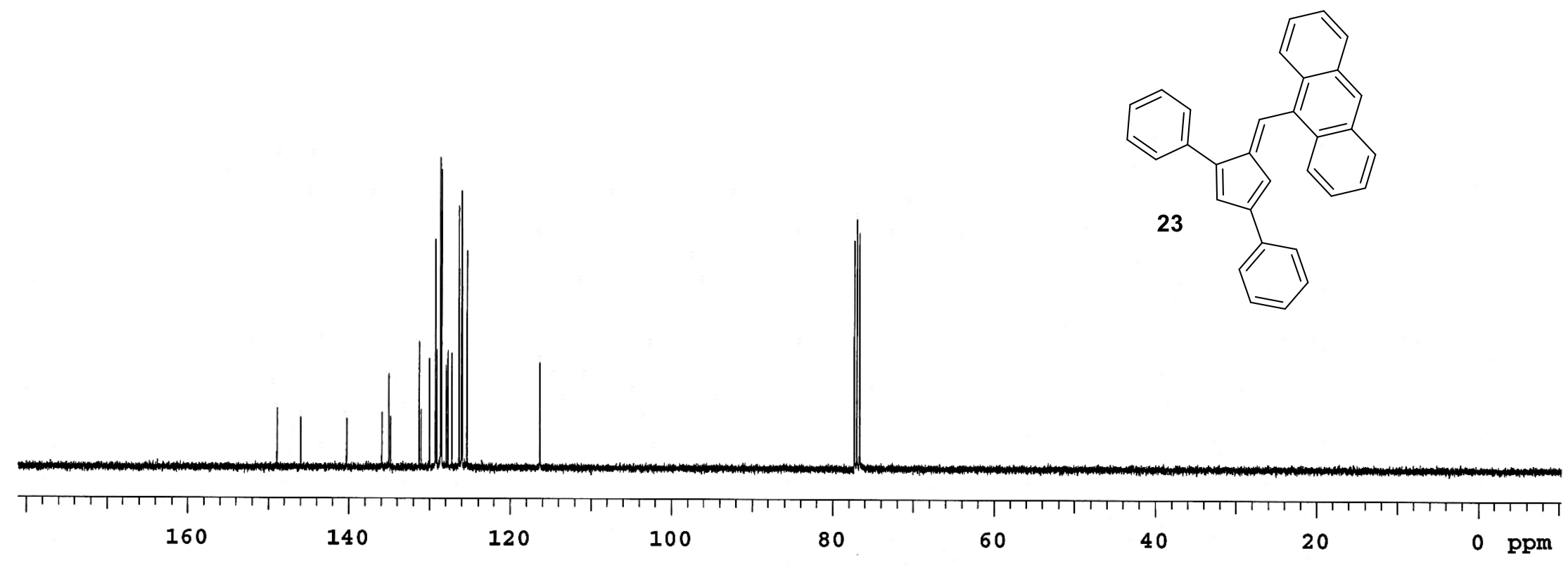

